BULLETIN (New Series) OF THE

AMERICAN MATHEMATICAL SOCIETY

Volume 41, Number 4, Pages 439-505

S 0273-0979(04)01035-3

Article electronically published on August 2, 2004

\title{
A TOUR OF THE THEORY OF ABSOLUTELY MINIMIZING FUNCTIONS
}

\author{
GUNNAR ARONSSON, MICHAEL G. CRANDALL, AND PETRI JUUTINEN
}

\begin{abstract}
These notes are intended to be a rather complete and self-contained exposition of the theory of absolutely minimizing Lipschitz extensions, presented in detail and in a form accessible to readers without any prior knowledge of the subject. In particular, we improve known results regarding existence via arguments that are simpler than those that can be found in the literature. We present a proof of the main known uniqueness result which is largely self-contained and does not rely on the theory of viscosity solutions. A unifying idea in our approach is the use of cone functions. This elementary geometric device renders the theory versatile and transparent. A number of tools and issues routinely encountered in the theory of elliptic partial differential equations are illustrated here in an especially clean manner, free from burdensome technicalities - indeed, usually free from partial differential equations themselves. These include a priori continuity estimates, the Harnack inequality, Perron's method for proving existence results, uniqueness and regularity questions, and some basic tools of viscosity solution theory. We believe that our presentation provides a unified summary of the existing theory as well as new results of interest to experts and researchers and, at the same time, a source which can be used for introducing students to some significant analytical tools.
\end{abstract}

\section{INTRODUCTION}

The subject of this paper is the study of existence, uniqueness and regularity properties of the best possible Lipschitz extensions of scalar functions. This seemingly elementary real analysis problem, described in detail below, conceals within itself a rich mathematical theory which has numerous applications and offers a technically uncomplicated passage to the world of fully nonlinear elliptic partial differential equations and modern calculus of variations. In fact, the extension problem and its associated Euler-Lagrange equation play the same fundamental role in the theory of calculus of variations of $L^{\infty}$ functionals as the classical Dirichlet integral and the Laplace equation do in the calculus of variations of $L^{2}$ functionals. Our approach to the extension problem, however, is not based on partial differential equations. On the contrary, we proceed more in the spirit of the problem itself, relying on very basic geometrical and analytical tools, and in a manner that does

Received by the editors July 18, 2003, and, in revised form, May 24, 2004.

2000 Mathematics Subject Classification. Primary 35J70, 49K20, 35B50.

"Absolutely minimizing" has other meanings besides the one herein. We might more properly say "absolutely minimizing in the Lipschitz sense" instead, but prefer to abbreviate.

The third author is supported by the Academy of Finland, project \#80566.

(C)2004 American Mathematical Society Reverts to public domain 28 years from publication 
not require heavy prerequisites or familiarity with any external theories. As a result, our arguments become versatile and can be readily extended to more general settings. Moreover, it will be clear that this approach bears a strong resemblance to the use of the mean value inequalities in connection with the study of harmonic functions, and we feel it adds a new, very geometric, aspect to the theory of calculus of variations of $L^{\infty}$ functionals and their Euler-Lagrange equations.

In order to provide the reader with a comprehensive and detailed view of the contents and methods of this paper, we must start from the very beginning. The origin of the theory we are about to present lies in the classical problem of extending Lipschitz continuous functions. To describe this extension problem in a simple setting, let us suppose we are given a function $f: \partial U \rightarrow \mathbb{R}$, defined on the boundary of a proper open subset $U$ of $\mathbb{R}^{n}$, such that its Lipschitz constant $\mathrm{L}_{f}(\partial U)$, that is, the least constant $L \in[0, \infty]$ for which

$$
|f(x)-f(y)| \leq L|x-y| \text { for } x, y \in \partial U
$$

holds, is finite. In (0.1), the "|.|" on the left is the absolute value in $\mathbb{R}$, while on the right it can be any given norm on $\mathbb{R}^{n}$. The task is to find a Lipschitz continuous extension, call it $u$, of $f$ to $U$ so that $u$ is continuous on the closure of $U$ and $\mathrm{L}_{u}(U)$ is as small as possible. Obviously, $\mathrm{L}_{u}(U)$ cannot be smaller than $\mathrm{L}_{f}(\partial U)$ since it is required that $u$ be a continuous extension of $f$, i.e., $u=f$ on $\partial U$. Thus the best one could hope for is $\mathrm{L}_{u}(U)=\mathrm{L}_{f}(\partial U)$, and this is indeed achieved by the explicit extensions

$$
\begin{aligned}
& \Psi(f)(x)=\inf _{y \in \partial U}\left(f(y)+\mathrm{L}_{f}(\partial U)|x-y|\right), \\
& \Lambda(f)(x)=\sup _{y \in \partial U}\left(f(y)-\mathrm{L}_{f}(\partial U)|x-y|\right),
\end{aligned}
$$

which may be found 1 in McShane [55] and Whitney [63. If $u$ is any solution of the extension problem, then $\Lambda(f) \leq u \leq \Psi(f)$; for this reason $\Psi(f)$ is called the maximal extension of $f$ and $\Lambda(f)$ is the minimal extension. The verifications of statements about $\Lambda(f), \Psi(f)$ made in this introduction are outlined in Section 1.2 below.

These extremal Lipschitz extensions fail to obey comparison and "stability" principles. More precisely, the relation $f_{1} \leq f_{2}$ on $\partial U$ does not, in general, imply either $\Psi\left(f_{1}\right) \leq \Psi\left(f_{2}\right)$ or $\Lambda\left(f_{1}\right) \leq \Lambda\left(f_{2}\right)$ in $U$, and $\Psi\left(\left.\Psi(f)\right|_{\partial V}\right)$ may differ from $\Psi(f)$ in an open subset $V$ of $U$. In particular, since it can happen that $\mathrm{L}_{\Psi(f)}(V)$ is larger than $\mathrm{L}_{\Psi(f)}(\partial V)$ for some $V$, a repeated application of the operator $\Psi$ (or $\Lambda$ ) in a subset can decrease the local Lipschitz constant. Hence it might be possible to "improve" the properties of an extension by applying either $\Psi$ or $\Lambda$ indefinitely.

The above discussion raises the following question: is it possible to find a "canonical" Lipschitz extension that would enjoy comparison and stability properties? And furthermore, could this special extension be unique once the boundary data is fixed? If such functions exist, they must satisfy

$$
\mathrm{L}_{u}(\partial V)=\mathrm{L}_{u}(V) \text { for any open } V \subset \subset U,
$$

because otherwise the stability would not hold: for example, the function $\tilde{u}$ which agrees with $u$ on $U \backslash V$ and with $\Lambda\left(\left.u\right|_{\partial V}\right)$ in $V$ would be "better" than $u$. This

\footnotetext{
${ }^{1}$ These extensions are also attributed to Banach [11] in [34, which also treats "localized" versions of Lipschitz continuity.
} 
observation, first appearing in the much cited works of Aronsson [3]-[5], can be taken as a starting point for a more refined study of the extension problem.

A continuous, real-valued function $u$ defined in $U$ is called absolutely minimizing if (0.2) holds for every $V \subset \subset U$, i.e., if $u$ has the least possible Lipschitz constant in every open set whose closure is compact and contained in $U$. Observe that in the definition of "absolutely minimizing", there is no a priori Lipschitz regularity assumed. Moreover, in the definition, any reference to a function to be extended has been removed, thereby initially decoupling the notion of minimization from the extension problem.

The McShane-Whitney operators provide a natural device for attempting to construct absolute minimizers since they can be used to reduce the Lipschitz constant in subdomains where it is not optimal. This idea does indeed work and it was used by Aronsson [5] to provide the first existence result for absolute minimizers. However, in these notes we will employ an even more concrete and elementary approach which can be motivated by the observation that the functions $\Psi(f)$ and $\Lambda(f)$ themselves are defined via geometrically very simple elements, "cones"; here the functions

$$
x \mapsto f(y) \pm \mathrm{L}_{f}(\partial U)|x-y| .
$$

Unlike the McShane-Whitney extensions, cones always satisfy (0.2) outside the vertex point, and they can be regarded as "fundamental solutions" of the problem. In this context, roughly speaking, the usual convolution with fundamental solutions is replaced by maximization and minimization procedures. Moreover, cones are defined purely in terms of the norm that is being used, and it is the norm that determines exact Lipschitz constants. Thus we are not introducing any ingredients not already present.

It turns out that all the absolute minimizers can be detected by checking the validity of a property that we call comparison with cones. The introduction of this notion helps us to streamline the existence theory and make it more transparent. In Section 3 we present a more general existence theorem than is currently available in the literature using this method. The uniqueness question is treated in Section 6 . Here, for the first time, the uniqueness results known to date are presented without invoking any substantial external theories. In this regard, the first uniqueness proof was given by Jensen [47, who used "viscosity solution" theory and the theory of Sobolev spaces, and the second by Barles and Busca [12, who used clever new arguments which circumvented the need to employ results on Sobolev spaces. The proof in Section [6 invokes neither viscosity solution theory nor results on Sobolev spaces. However, it amounts to introducing the relevant tools of viscosity solution theory, along with analogues of key arguments of 12, without introducing the viscosity solution theory itself. This rendering, which we hope will make the proof widely accessible, is possible due to the use of basic results obtained herein for functions that enjoy comparison with cones.

The discussion above has been quite elementary. However, it turns out that the problem it introduces provides an archetypal example for more general theories. This greatly enhances interest in understanding it in all possible detail. In particular, there is an associated degenerate elliptic equation, which is as yet poorly understood, even in the case of the Euclidean norm. Moreover, the problem is also an archetype in the theory of minimization of $L^{\infty}$ functionals. We sketch these connections below. 
Since the works [3]-[5], the theory of absolute minimizers has been advanced by a number of authors. The most popular line of research has risen from the idea, again due to Aronsson [5], of interpreting the Lipschitz extension problem as a formal limit, as $p \rightarrow \infty$, of the more classical variational problem of minimizing the functional

$$
I_{p}(v, U)=\int_{U}|D v|^{p} d x, \quad 1<p<\infty
$$

under given boundary conditions; see, e.g., [18, [47. Here $D v=\left(v_{x_{1}}, \ldots, v_{x_{n}}\right)$ denotes the gradient of $v$. This approach, in the case of the Euclidean norm, leads to a reformulation of the original problem in which $(0.2)$ is replaced by the requirement

$$
\underset{x \in V}{\operatorname{ess} \sup }|D u(x)| \leq \underset{x \in V}{\operatorname{ess} \sup }|D v(x)|
$$

for all $V \subset \subset U$ and "sufficiently regular" $v$ satisfying $v=u$ on $\partial V$. Indeed, minimizing $I_{p}(v, U)$ (subject to $v=f$ on $\partial U$ ) is the same as minimizing the $L^{p}$ norm of $|D v|$, which tends to the $L^{\infty}$ norm as $p \rightarrow \infty$. Due to the set additivity of $I_{p}$, a minimizer of $I_{p}(\cdot, U)$ also minimizes $I_{p}(\cdot, V)$ for any $V \subset \subset U$ (subject to its own boundary values), and hence it is automatically an "absolute minimizer" of (0.3). It is natural to require that this should hold also in the limit case, and that is precisely what is stated in (0.4). However, it is not clear at all that (0.4) is equivalent to (0.2). If $U$ is convex, then

$$
\mathrm{L}_{v}(U)=\underset{U}{\operatorname{ess} \sup }|D v|
$$

for any locally Lipschitz continuous function $v$. But if $U$ is not convex, then $L_{v}(U)$

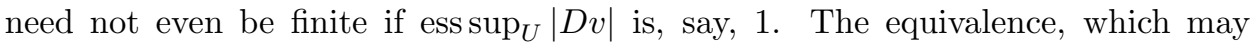
not be generally appreciated, is established in Section 4 without reference to the approximation by the functional $I_{p}$. Again, in the discussion above, it was assumed that $|\cdot|$ is the Euclidean norm, as it was in the references mentioned. See Section 8 concerning other norms.

The approximation procedure discussed above has the advantage of providing a route to the correct "Euler-Lagrange" equation for our problem. Namely, a function $u$ for which $I_{p}(u, U) \leq I_{p}(v, U)$ if $v=u$ on $\partial U$ should satisfy

$$
\lim _{t \rightarrow 0} \frac{I_{p}(u+t \phi, U)-I_{p}(u, U)}{t}=0
$$

for smooth functions $\phi$ vanishing off a compact subset of $U$. This leads to the "p-Laplace" equation

$$
\Delta_{p} u:=\text { divergence }\left(|D u|^{p-2} D u\right)=0,
$$

which is to be understood in the sense of distributions or in the viscosity sense. Notice that the classical case $p=2$ yields the familiar Laplace equation $\Delta u=0$, whose solutions are the harmonic functions. Taking the limit of (0.6) as $p \rightarrow \infty$, a somewhat subtle matter, one obtains a nonlinear and highly degenerate elliptic equation,

$$
\Delta_{\infty} u:=\sum_{i, j=1}^{n} u_{x_{i}} u_{x_{j}} u_{x_{i} x_{j}}=0 \text { in } U
$$


known as the infinity Laplace equation. We derive this equation in Section 4.4 without reference to the approximation procedure, instead starting from the purely geometric property of comparison with cones. The infinity Laplace equation played a crucial role in the acclaimed original proof of uniqueness by Jensen [47], and it has become a center of attention in this field. The price that has to be paid in this approach is the loss of generality. The original extension problem makes sense in any metric space, as do the cones.

In Section 4.4 we show that absolutely minimizing functions are precisely the viscosity solutions of the infinity Laplace equation in the case of the Euclidean norm. The appropriate equations are derived for other norms in Section 5 . The issue of the equivalence between "absolutely minimizing" and viscosity solutions of an appropriate partial differential equation is not completely settled for general norms. We mention again that uniqueness of absolutely minimizing extensions of a function given on the boundary of a bounded open set into the set is proved for the Euclidean norm in Section [6 without relying on the equivalence of absolutely minimizing functions and viscosity solutions of (0.7).

Apart from the nice geometric interpretation, the theory of absolute minimizers is interesting because it serves as an archetypal example of a problem in the calculus of variations involving $L^{\infty}$ functionals of the form

$$
\underset{x \in \Omega}{\operatorname{ess} \sup } F(x, u, D u) .
$$

This connection is evident from (0.4). These variational problems arise in many contexts and provide often the most realistic framework for concrete physical problems. See, e.g., [13] for a list of possible applications. The systematic mathematical study of $L^{\infty}$ functionals has been greatly influenced by the advances in understanding the Lipschitz extension problem, and vice versa. Aronsson's original work [5] appeared after he had investigated $L^{\infty}$ functionals in the one dimensional case in [3] and [4. Recent progress in the theory of general $L^{\infty}$ functionals in [13], [14, [28] and [65, as well as the treatment of some special examples in [50, [39], [44] and [15], follow in spirit the decisive contribution of Jensen [47] on the Lipschitz extension problem.

As a concrete application, we mention that absolutely minimizing extensions have attracted some interest in image processing, beginning with papers by Caselles, Morel and Sbert; see, e.g., 25. The main usage of absolute minimizers here seems to be for the interpolation of pictures with incomplete information (incomplete or irregular sampling). This so-called AMLE model has attractive properties of invariance, monotonicity and stability, essential for these applications. A recent contribution to this field is [1, where the AMLE model is used for interpolation of terrain elevation maps. These maps can, for instance, be obtained from satellites. Comparisons are made with other methods of interpolation and the outcome is favorable for the AMLE approach. Another application area in which these ideas have been employed is the analysis of possible shapes of sandpiles; cf. [10] and [2].

Let us recapitulate the contents of these notes. We begin in Section 1 with a more detailed discussion of the classical Lipschitz extension problem and absolute minimizers, and substantiate the claims made in the beginning of this introduction. Section 2 deals with the comparison with cones property. We show that it characterizes absolute minimizers and use it to obtain important a priori estimates. Given these lemmas, the proof of our existence result in Section 3 is rather 
straightforward and simple. In Section 4 we turn to the equivalence of various notions. In particular, as a special case of our results for general norms, we verify that conditions (0.2) and (0.4) yield the same class of functions. This is done with the aid of the auxiliary concept of strong absolute minimizers, which we define as part of the preliminaries in Section 1.5. A new derivation of the infinity Laplace equation (0.7) from the comparison with cones property is given, and subsequently we prove that its viscosity solutions coincide with the absolute minimizers. A variety of other equivalences are also proved. In particular, it is shown that, for all norms, a function is absolutely minimizing in a set whenever every point has a neighborhood in which this is true. That is, the concept is entirely local, a fact which is not obvious. In Section 5 the analogue of the infinity Laplacian is derived for general norms. Section 6 contains the proof of the uniqueness theorem. For the convenience of the reader, all of its main ingredients are thoroughly discussed and the presentation is virtually self-contained. Finally, in Section 7 , the largely open issue of the exact regularity of absolute minimizers is reviewed and known positive results are presented.

We made several allusions to the "Euclidean norm" above. Let us clarify the situation. In all of the results in the body of this work, the norm is "general" with the following exceptions, which are presented for the case of the Euclidean norm: the equivalence with viscosity solutions in Section 4.4 the uniqueness result of Section 6] and the regularity result of Section 7. The results of Section 4.4 (properly adapted), and Section [6 hold if the norm $|\cdot|$ is strictly convex, and the main result (Theorem [7.1) of Section 7 is true if $|\cdot|$ is strictly convex and differentiable on the complement of the origin. However, we preferred to make these results available without requiring the reader to deal with the additional issue of the strict convexity of norms and dual norms. It is, as of the moment, unsettled as to whether or not the results of Section 4.4 (appropriately formulated) and Section $[6$ hold in general. The results of Section 7 are false for general norms.

For completeness and the reader's convenience, we have also included a short appendix, Section 8, on the associated variational problems in $L^{p}$ and the approximation procedure to which we referred above, and another on absolute minimizers in general metric spaces, Section 9 The presentation in these last two sections is somewhat formal and less self-contained than in the main text, with the aim of giving the reader a rough idea of the nature of these topics.

Every section ends with a set of notes and comments. There we have included a brief historical account of each of the topics treated in the section as well as some relevant references; references are minimized in the main text. However, we do not provide pointers to all of the papers listed in the bibliography, which we have made as complete as we could for the reader's convenience. Although we have made a serious effort to find significant related papers and present each work properly, we cannot claim that this introduction, the list of references and the notes ending each section have completely succeeded in describing the history and the current status of this beautiful theory.

We are indebted to Carl de Boor and Simeon Reich for suggestions which improved this manuscript. 


\section{Contents}

Introduction 439

1. Notation and other preliminaries 446

1.1. Notation 446

1.2. The Lipschitz Extension Problem 447

1.3. Absolutely Minimizing Functions and Another Extension Problem 448

1.4. Cone Functions 448

1.5. Another Kind of Absolutely Minimizing Function 449

1.6. Notes 450

2. Comparison with cones 450

2.1. The Notions and the Equivalence 451

2.2. First Consequences 452

2.3. Local Maxima Imply Locally Constant

2.4. Second Consequences 456

2.5. The Increasing Slope Estimate 458

2.6. A Sharper Harnack Inequality 4459

2.7. Notes 460

3. Existence 461

3.1. Notes 463

4. Equivalences 464

4.1. Formulation of the Equivalences 464

4.2. Proof of the One-Sided Equivalences (a)-(f) 467

4.3. Proof of the Two-Sided Equivalences (a)-(f) 470

4.4. Viscosity Solutions and the Infinity Laplace Operator in the Euclidean

4.5. Notes

5. The infinity Laplace operator in general norms

5.1. Notes 479

6. The uniqueness question 480

6.1. Properties of Sup-Convolution 4483

6.2. Elements of Convex Analysis 484

6.3. The End Game 486

6.4. Notes 489

7. Regularity 490

7.1. Notes 493

8. Appendix: $L^{p}$ variational problems 493

8.1. Sobolev spaces 493

8.2. $p$-harmonic functions 494

8.3. Connection to Lipschitz extensions 495

8.4. Euler-Lagrange equations 496

8.5. Notes 497

9. Appendix: Absolute minimizers in general metric spaces 497

9.1. Notes 502

References $\quad 502$ 


\section{Notation AND Other PRELIMINARIES}

It will be assumed in succeeding sections that the reader is familiar with the material of this section except as noted. The presentation is informal and will be called on later via the phrase "by Section 1.m" (rather than "by Theorem 1.j", etc.). Concepts introduced above are revisited, displayed and discussed in more detail.

1.1. Notation. Throughout this paper $U, V, W$ always denote open subsets of $\mathbb{R}^{n}$; $\partial U$ denotes the boundary of $U$ and $\bar{U}$ is the closure of $U$. The coordinates of $x \in \mathbb{R}^{n}$ are denoted by $x_{j} ; x=\left(x_{1}, x_{2}, \ldots, x_{n}\right)$. The notation $V \subset \subset U$ means that $\bar{V}$ is a compact subset of $U$.

If $a \in \mathbb{R}$, then $|a|$ is the absolute value of $a$. If $x \in \mathbb{R}^{n}$, then $|x|$ is the "norm" of $x$. We did not say "Euclidean norm", for $|\cdot|$ can in fact be any norm on $\mathbb{R}^{n}$ in most of this work. However, most of the time the reader can assume that $|\cdot|$ is the Euclidean norm if it pleases the reader to do so.

An expression of the form $Y:=X$ indicates that $Y$ is defined to be $X$. If $x \in \mathbb{R}^{n}$, then

$$
\operatorname{dist}(x, \partial U):=\inf \{|x-y|: y \in \partial U\}
$$

is the distance from $x$ to $\partial U$.

If $x, y \in \mathbb{R}^{n}$, then

$$
\langle x, y\rangle:=\sum_{j=1}^{n} x_{j} y_{j}
$$

is the usual inner-product of $x$ and $y$. It is not true that $\langle x, x\rangle=|x|^{2}$ if $|\cdot|$ is not the Euclidean norm. However, if $|\cdot|^{*}$ is the norm dual to $|\cdot|$, that is,

$$
|x|^{*}:=\max \left\{\langle x, y\rangle: y \in \mathbb{R}^{n},|y|=1\right\},
$$

then

$$
|\langle x, y\rangle| \leq|x|^{*}|y|
$$

The open ball of radius $r$ and center $x \in \mathbb{R}^{n}$ is denoted by $B_{r}(x)$ :

$$
B_{r}(x):=\left\{y \in \mathbb{R}^{n}:|x-y|<r\right\} ;
$$

note that this ball is defined via the metric induced by the norm in use.

If $K \subset \mathbb{R}^{n}$ and $g: K \rightarrow \mathbb{R}$, then $\mathrm{L}_{g}(K)$ is the least constant $L \in[0, \infty)$ for which

$$
|g(x)-g(y)| \leq L|x-y| \text { for } x, y \in K \text {. }
$$

If there is no such constant $L$, we put $\mathrm{L}_{g}(K)=\infty$. If $\mathrm{L}_{g}(K)<\infty$, then $g$ is Lipschitz continuous (on $K$ ). If $L_{g}(K) \leq L$, then $L$ is "a Lipschitz constant for $g$ ".

The set of continuous real-valued functions on $K$ is denoted by $C(K) . C^{k}(U)$ is the subset of $C(U)$ consisting of those functions which are $k$ times continuously differentiable in $U$.

If $X, Y$ are real, symmetric $n \times n$ matrices, then $X \geq Y(X>Y)$ means $\langle X x, x\rangle \geq\langle Y x, x\rangle$ (respectively, $\langle X x, x\rangle>\langle Y x, x\rangle$ ) for $x \in \mathbb{R}^{n} \backslash\{0\}$.

The line segment joining two points $w, z \in \mathbb{R}^{n}$ is denoted by $[w, z]$ :

$$
[w, z]:=\{w+t(z-w): 0 \leq t \leq 1\} .
$$


1.2. The Lipschitz Extension Problem. Let $K \subset \mathbb{R}^{n}$ and $f: K \rightarrow \mathbb{R}$ be Lipschitz continuous. In contrast with the introduction, $K$ need not be the boundary of an open set $U$. If " $f$ " represents some partial information available only on $K$ and we want to infer information outside of $K$, that is, we seek to extend $f$ to $\mathbb{R}^{n} \backslash K$, it seems reasonable to ask if this can be done without increasing the Lipschitz constant. That is, we ask if there exists a $u \in C\left(\mathbb{R}^{n}\right)$ satisfying

$$
u=f \quad \text { on } \quad K \quad \text { and } \quad \mathrm{L}_{u}\left(\mathbb{R}^{n}\right)=\mathrm{L}_{f}(K) .
$$

If $y, z \in K, x \in \mathbb{R}^{n}$, then any such $u$ clearly satisfies

$$
f(z)-\mathrm{L}_{f}(K)|x-z| \leq u(x) \leq f(y)+\mathrm{L}_{f}(K)|x-y|
$$

since $f(z)=u(z), f(y)=u(y)$. Moreover, if $x \in K$, these two inequalities become equalities upon choosing $z=y=x$. We add two more simple observations to the preceding ones:

(i) $f(z)-\mathrm{L}_{f}(K)|x-z| \leq f(y)+\mathrm{L}_{f}(K)|x-y|$ for $x \in \mathbb{R}^{n}, y, z \in K$.

(ii) The infimum and supremum of a family of functions with a fixed Lipschitz constant have, if finite, the same Lipschitz constant.

Combining these observations, one concludes that

$$
\begin{aligned}
& \Lambda(f)(x):=\sup _{y \in K}\left(f(y)-\mathrm{L}_{f}(K)|x-y|\right) \\
& \Psi(f)(x):=\inf _{y \in K}\left(f(y)+\mathrm{L}_{f}(K)|x-y|\right)
\end{aligned}
$$

define solutions $\Lambda(f), \Psi(f)$ of (1.2) such that if $u$ is any other solution, then $\Lambda(f) \leq$ $u \leq \Psi(f)$ in $\mathbb{R}^{n}$. Clearly, solutions of (1.2) are unique if and only if $\Lambda(f)=\Psi(f)$ on $\mathbb{R}^{n}$. This rarely happens.

Example 1.1. Let $n=1, K=\{-1,0,1\}, f(-1)=f(0)=0, f(1)=1$. Then $\mathrm{L}_{f}(K)=1$. A moment's thought shows that $\Psi(f), \Lambda(f)$ are as indicated in Figure 1.1
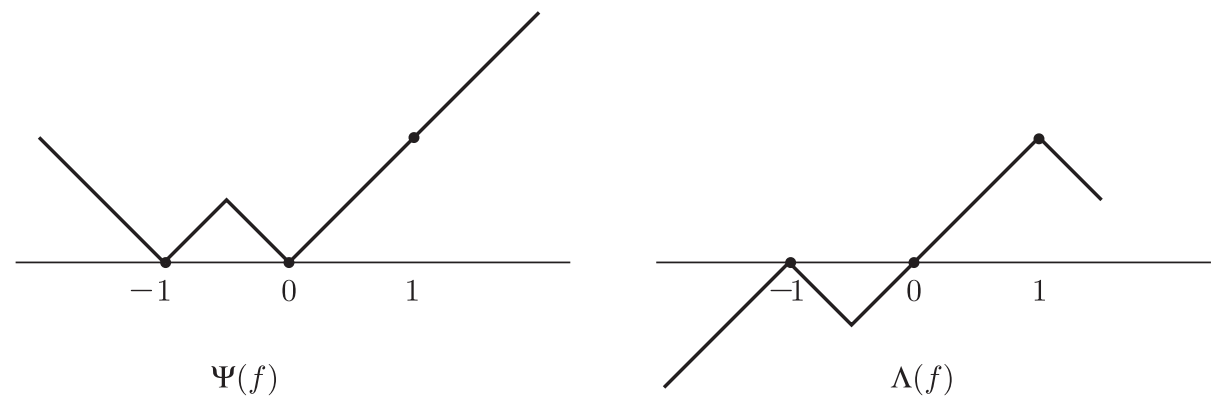

FiguRe 1.1.

Remark 1.2. In Section 4 we will have occasion to use the notations $\Lambda(f), \Psi(f)$ when $f$ is not necessarily Lipschitz continuous. In this case we put $\Lambda(f) \equiv-\infty$ and $\Psi(f) \equiv+\infty$. 


\subsection{Absolutely Minimizing Functions and Another Extension Problem.} Clearly, there are many other solutions of (1.2) in Example 1.1 besides the ones pictured; maybe the "best" one is given by

$$
u(x)=0 \text { for } x \leq 0, \quad u(x)=x \text { for } 0 \leq x \leq 1 \text {, and } u(x)=1 \text { for } 1 \leq x .
$$

Why do we prefer this solution? Roughly speaking, because it locally varies "as little as possible" among all the potential solutions. In particular, it has the property (0.2), which we restate here: if $V \subset \subset \mathbb{R} \backslash K$, then

$$
\mathrm{L}_{u}(V)=\mathrm{L}_{u}(\partial V) .
$$

The reader should think through this claim. Note that neither $\Lambda(f)$ nor $\Psi(f)$ has this property, while the function $\tilde{u}(x)=0$ if $x \leq 0$ and $\tilde{u}(x)=x$ if $0 \leq x$ is a second extension of $f$ with the same Lipschitz constant and the property (1.3). Moreover, $\hat{u}(x)=0$ if $x \leq 0, \hat{u}(x)=x$ if $0 \leq x \leq 1, \hat{u}(x)=2 x-1$ if $x \geq 1$ is another function agreeing with $f$ on $K$ which satisfies (1.3), but it does not satisfy $\mathrm{L}_{u}(\mathbb{R})=\mathrm{L}_{f}(K)$.

We formalize the definition of "absolutely minimizing":

Definition 1.3. Let $U \subset \mathbb{R}^{n}$. Then $u \in \operatorname{AM}(U)$ if $u \in C(U)$ and (1.3) holds for every $V \subset \subset U$.

Here "AM" stands for "absolutely minimizing". The modifier "absolutely" refers to the arbitrary choice of $V$ in (1.3). It is, of course, the Lipschitz constant that is being minimized, in the sense that it is as small as it can be on each $V$, given the values on $\partial V$.

The example above also illustrates another remark made in the introduction. If $g$ is such that $g(-1)=0,0<g(0)<1$, and $g(1)=1$, then the reader can quickly verify that $\Psi(f) \not \leq \Psi(g) \not \leq \Psi(f)$ even though $f \leq g$.

Looking at the simple picture above, it seems natural to consider also the extension problem from $K$ to the interval $[-1,1]$ rather than to all of $\mathbb{R}$, as we may only be interested in the interval. This leads to the following problem: let $U \subset \mathbb{R}^{n}$ and $f \in C(\partial U)$. Find $u$ with the following properties:

$$
u \in C(\bar{U}) \cap \operatorname{AM}(U) \text { and } u(x)=f(x) \text { for } x \in \partial U \text {. }
$$

Note that (BVP) does not assume that $f$ itself is Lipschitz continuous. This is one of the reasons that the relationship between our original remarks about the Lipschitz extension problem (1.2) and (BVP) were not entirely clear. If the $f$ in (BVP) is Lipschitz continuous and $\mathbb{R}^{n}=U \cup \partial U$, does a solution of (BVP) also solve (1.2)? Not in general; we gave an example above. However, if $U$ is bounded, we will see in Section 4 that solutions of $(\mathrm{BVP})$ do satisfy $\mathrm{L}_{u}(U)=\mathrm{L}_{f}(\partial U)$.

1.4. Cone Functions. A cone function is one of the form $C(x):=a|x-z|+b$ where $a, b \in \mathbb{R}$ and $z \in \mathbb{R}^{n}$. We say that $a$ is the slope of $C$ and $z$ is the vertex of $C$. The McShane-Whitney extensions are formed by infs and sups over cone functions with vertices in the set where function values are given, a fact which hints at their utility.

Cone functions provide very important examples of absolutely minimizing functions. We show that if $C$ is a cone function with vertex $z$, then

$$
C \in \operatorname{AM}\left(\mathbb{R}^{n} \backslash\{z\}\right) .
$$

This follows from the observations that $|a|=\mathrm{L}_{C}\left(\mathbb{R}^{n}\right)$ and that for any $W \subset\left(\mathbb{R}^{n} \backslash\right.$ $\{z\})$ and $y \in W$, we can choose two distinct points $y^{*}, y^{* *} \in \partial W$ located on the 


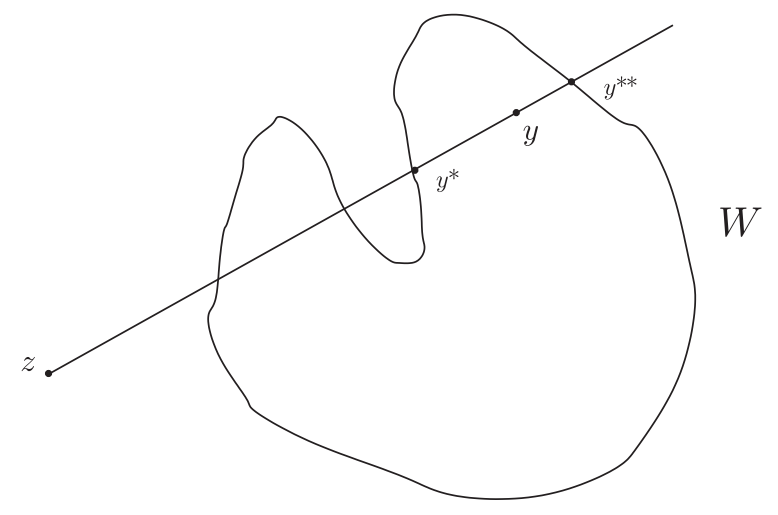

FIGURE 1.2.

half-line with end point $z$ passing through $y$ so that the Lipschitz constant of $C$ restricted to these two points is also $|a|$. The picture is as shown in Figure 1.2, One has $\left|C\left(y^{*}\right)-C\left(y^{* *}\right)\right|=|a|\left|y^{* *}-y^{*}\right|$.

Cone functions also have the following property: if $W \subset \subset \mathbb{R}^{n}, C$ is a cone function with vertex $z \notin W$ and slope $a$, and $u \in C(\bar{W})$ satisfies $u=C$ on $\partial W$ and $\mathrm{L}_{u}(W)=|a|$, then $u \equiv C$ on $W$. To establish this, we show that $y \in W$ and $u(y) \neq C(y)$ is impossible. To rule out $u(y)>C(y)$ when $a \geq 0$, assume it and let $y^{*}, y^{* *}$ be as in Figure 1.2. Then

$$
u(y)-u\left(y^{*}\right)=u(y)-C\left(y^{*}\right)>C(y)-C\left(y^{*}\right)=a\left|y-y^{*}\right|,
$$

which shows that $\mathrm{L}_{u}(W)>a$, a contradiction. If $a<0$, one uses the point $y^{* *}$ in place of $y^{*}$ in a similar way. To rule out $u(y)<C(y)$, the previous argument applies to $-u,-C$.

Cone functions will play a prominent role in the rest of this paper.

1.5. Another Kind of Absolutely Minimizing Function. The material in this subsection is not needed until Section 4 . The reader may skip it until it is needed.

If $u \in C(U)$, the function

$$
\mathrm{T}_{u}(x):=\lim _{r \downarrow 0} \mathrm{~L}_{u}\left(B_{r}(x)\right)=\inf \left\{\mathrm{L}_{u}\left(B_{r}(x)\right): 0<r<\operatorname{dist}(x, \partial U)\right\}
$$

is well-defined on $U$ if we allow the value $+\infty$.

Roughly speaking, $\mathrm{T}_{u}(x)$ is the Lipschitz constant of $u$ "at $x$ ". It is the smallest number with the property that for $\epsilon>0$ there is an $r_{\epsilon}>0$ such that $\mathrm{T}_{u}(x)+\epsilon$ is a Lipschitz constant for $u$ on $B_{r_{\epsilon}}(x)$. We claim that $x \rightarrow \mathrm{T}_{u}(x)$ is uppersemicontinuous. To see this, note that $\mathrm{T}_{u}(x)<M$ implies $\mathrm{L}_{u}\left(B_{r}(x)\right)<M$ for some $r>0$. For $y \in B_{r}(x)$ and $s+|x-y| \leq r$, we have $B_{s}(y) \subset B_{r}(x)$ and then $\mathrm{L}_{u}\left(B_{s}(y)\right) \leq \mathrm{L}_{u}\left(B_{r}(x)\right)$. Hence $\mathrm{T}_{u}(y)<M$. This shows that $\left\{x \in U: \mathrm{T}_{u}(x)<M\right\}$ is open, establishing the upper-semicontinuity.

Definition 1.4. Let $u \in C(U)$. Then $u$ is strongly absolutely minimizing (equivalently, $u \in \operatorname{AMS}(U))$ if for every $V \subset \subset U$ and $v \in C(\bar{V})$ such that $u=v$ on $\partial V$, one has

$$
\sup _{x \in V} \mathrm{~T}_{u}(x) \leq \sup _{x \in V} \mathrm{~T}_{v}(x)
$$


It will be seen in Section 4 that $\operatorname{AM}(U)=\operatorname{AMS}(U)$. The inclusion $\operatorname{AMS}(U) \subset$ $\operatorname{AM}(U)$ is relatively easy to establish, while the inclusion $\operatorname{AM}(U) \subset \operatorname{AMS}(U)$ is more difficult. This is the reason for our use of the descriptor "strong".

The function $\mathrm{T}_{u}$ is closely related to the gradient $D u$ of $u$. We say that $u$ is differentiable at $x_{0} \in U$ if there exists $p \in \mathbb{R}^{n}$ such that

$$
u(x)=u\left(x_{0}\right)+\left\langle p, x-x_{0}\right\rangle+\mathrm{o}\left(\left|x-x_{0}\right|\right)
$$

as $x \rightarrow x_{0}$. Here the "little o" notation has its usual meaning. When (1.5) holds, then $p$ is clearly uniquely determined, and we write $D u\left(x_{0}\right):=p$. That is, $D u\left(x_{0}\right)$ is the one and only vector providing a first order Taylor expansion for $u$ around $x_{0}$ when there is such an expansion. By Rademacher's theorem (see, e.g., [36]), Lipschitz continuous functions are differentiable almost everywhere in this sense. We leave it as an exercise for the reader to show that if $V \subset U$, then

$$
\sup _{x \in V} \mathrm{~T}_{u}(x)=\operatorname{ess}_{x \in V}|D u(x)|^{*}
$$

whenever the left-hand side is finite; here $|\cdot|^{*}$ is the norm dual to $|\cdot|$ as explained in Section 1.1 above.

1.6. Notes. The idea of "absolute minimizers" first arose in Aronsson 3], 4, where it appeared in the study of certain minimization problems for functionals of the type

$$
I(f)=\sup _{x} F\left(x, f(x), f^{\prime}(x)\right) .
$$

In connection with the Lipschitz extension problem, it was introduced in [5] in the form used in this work. Various adaptations of the concept have since been used in [18], [47, 64], [57, [49] and [29] to investigate the extension problem in different settings. Moreover, an "absolute minimizer" has become a standard notion of a minimizer in the theory of the calculus of variations of $L^{\infty}$ functionals; see [13], [14], 28], [27] and [65] and the references therein.

Our formulation of the notion of a strong absolute minimizer appears explicitly for the first time in this work. It is essentially a reformulation of (0.4) written in a simpler and more general form, without the aid of measure and differentiation theory.

Needless to say, the Lipschitz extension problem makes sense in much wider generality than described above. For example, one can investigate mappings $u$ : $X \rightarrow Y$ between two metric spaces $\left(X, d_{X}\right)$ and $\left(Y, d_{Y}\right)$ and pose the same questions asked above regarding existence and uniqueness of various types of extensions. The case in which the target space $\left(Y, d_{Y}\right)$ is $\mathbb{R}$ equipped with the Euclidean metric is, as regards existence, fairly well understood; see [57, [49. This is mainly due to the fact that the McShane-Whitney extensions and Perron's method work just as well in this generality; see Section 9. However, far less is known in the vector-valued case $Y=\mathbb{R}^{n}, n \geq 2$, with the Euclidean distance. A famous theorem of Kirszbraun (see, e.g., 37]) provides an extension of a given Lipschitz function with the same Lipschitz constant in this case. However, as far as we know, there are no existence results for absolute minimizers in the vector-valued case except when $X=\mathbb{R}$, which is treated by Barron, Jensen and Wang 13 .

\section{COMPARISON WITH CONES}

We begin this section by rewriting the condition that $u$ be absolutely minimizing in terms of another concept, called "comparison with cones". After defining the 
notions of comparison with cones and examining the relationships between these and the concept of an absolutely minimizing function, we establish consequences of comparison with cones that are prominent tools in the rest of this work. In particular, a small selection of results from Section 2.2 as indicated therein, is all that is needed for the proof of the existence theorem in Section 3. With these preliminaries in hand, the existence proof is short and elegant. The remaining results of Section 2.2 and Sections 2.3, 2.4 and 2.5 play roles in Sections 4, 6] and 7 Taken together, the results of this section provide the known fundamental estimates that flow from comparison with cones.

2.1. The Notions and the Equivalence. We begin by deriving some properties of $u \in \operatorname{AM}(U)$. These properties are then used to define a class of functions, and subsequently it is shown that this class coincides with $\operatorname{AM}(U)$. Moreover, this equivalence naturally splits "absolutely minimizing" into the conjunction of two "one-sided" notions, which is essential for the existence proof in Section 3

Proposition 2.1. Let $u \in C(U)$. Then $u \in \operatorname{AM}(U)$ iff for every $V \subset \subset U, a \in \mathbb{R}$ and $z \in \mathbb{R}^{n} \backslash V$ we have

$$
u(x)-a|x-z| \leq \max _{w \in \partial V}(u(w)-a|w-z|) \text { for } x \in V
$$

and

$$
u(x)-a|x-z| \geq \min _{w \in \partial V}(u(w)-a|w-z|) \text { for } x \in V .
$$

The following terminology will be much used.

Definition 2.2. If $u \in C(U)$, then $u$ enjoys comparison with cones from above (respectively, below) in $U$ if (2.1) (respectively, (2.2)) holds when $V \subset \subset U, a \in \mathbb{R}$ and $z \notin V$. If $u \in C(U)$ and enjoys comparison with cones from above and from below in $U$, then $u$ enjoys comparison with cones in $U$.

For simplicity in writing, we often abbreviate " $u$ enjoys comparison with cones from above (below) in $U$ " to

$$
u \in \mathrm{CCA}(U) \text { and, respectively, } u \in \mathrm{CCB}(U) .
$$

Note that $u \in \mathrm{CCA}(U)$ iff $-u \in \operatorname{CCB}(U)$. Of course,

$$
\mathrm{CC}(U)=\operatorname{CCA}(U) \cap \mathrm{CCB}(U) \text {. }
$$

Here is the proof of Proposition 2.1,

Proof. First, let $V \subset \subset U, x \in V$, and $u \in C(U)$ enjoy comparison with cones in $U$. We claim then that

$$
\mathrm{L}_{u}(\partial(V \backslash\{x\}))=\mathrm{L}_{u}(\partial V) .
$$

To see this, we need only check that for $z \in \partial V, x \in V$,

$$
u(z)-\mathrm{L}_{u}(\partial V)|x-z| \leq u(x) \leq u(z)+\mathrm{L}_{u}(\partial V)|x-z| .
$$

As each of the above inequalities holds for $x \in \partial V$ and $u$ enjoys comparison with cones, the inequalities indeed hold if $x \in V$. Let $x, y \in V$. Using (2.3) twice,

$$
\mathrm{L}_{u}(\partial V)=\mathrm{L}_{u}(\partial(V \backslash\{x\}))=\mathrm{L}_{u}(\partial(V \backslash\{x, y\})) .
$$

Since $x, y \in \partial(V \backslash\{x, y\})$, we have $|u(x)-u(y)| \leq \mathrm{L}_{u}(\partial V)|x-y|$, and hence $u \in \operatorname{AM}(U)$. 
Suppose now that $u \in \operatorname{AM}(U)$. We want to show that $u$ then enjoys comparison with cones from above. Assume that $V \subset \subset U, z \notin V$ and set

$$
W=\left\{x \in V: u(x)-a|x-z|>\max _{w \in \partial V}(u(w)-a|w-z|)\right\} .
$$

We want to show that $W$ is empty. If it is not empty, then it is open and

$$
u(x)=a|x-z|+\max _{w \in \partial V}(u(w)-a|w-z|)=: C(x) \text { for } x \in \partial W .
$$

Therefore $u=C$ on $\partial W$ and $\mathrm{L}_{u}(W)=\mathrm{L}_{C}(\partial W)$ since $u \in \operatorname{AM}(U)$. But then $u=C$ in $W$ by Section 1.4, which is a contradiction.

The claim concerning comparison with cones from below follows upon applying the previous case to $-u$.

Remark 2.3. We note that in the proof above it was shown that if $u$ does not enjoy comparison with cones from above in $U$, then there is a nonempty set $W \subset \subset U$ and a cone function $C(x)=a|x-y|+b$ with $y \notin W$ such that $u=C$ on $\partial W$ and $u>C$ in $W$. The negation of $u \in \operatorname{CCB}(U)$ has the corresponding formulation.

Example 2.4. It follows from (1.4) and Proposition 2.1 that if $u(x):=\tilde{a}|x-y|$, then $u$ enjoys comparison with cones in $\mathbb{R}^{n} \backslash\{y\}$. For brevity, we simply say "cones enjoy comparison with cones" to refer to this fact.

In fact, if $\tilde{a} \geq 0$, then $u$ enjoys comparison with cones from above in $\mathbb{R}^{n}$. We may suppose that $\tilde{a}>0$. If the assertion were false, then by Remark 2.3 there would exist a nonempty set $W \subset \subset \mathbb{R}^{n}$ and a cone function $C(x)=a|x-z|+b$ with $z \notin W$ such that $\tilde{a}|x-y|=C(x)$ on $\partial W$ and $\tilde{a}|x-y|>C(x)$ in $W$. We already know this is impossible if $y \notin W$. If $y \in W$, let $y^{*}, y^{* *}$ be as in Figure 1.2 Assume that $a \geq 0$; then, using $y^{*} \in \partial W, \tilde{a}>0$, and $|y-z|>\left|y^{*}-z\right|$, we have $C(y) \geq C\left(y^{*}\right)$ and

$$
0=\tilde{a}\left|y^{*}-y\right|-C\left(y^{*}\right)>\tilde{a}|y-y|-C(y),
$$

which contradicts $y \in W$. If $a<0$, we select $y^{* *}$ in place of $y^{*}$ and reach a similar conclusion.

Observe that if $\tilde{a}>0$, then $u$ does not enjoy comparison with cones from below in any open set containing the vertex $y$. On the other hand, if $\tilde{a} \leq 0$, applying the above to $-u$ we see that $u$ enjoys comparison with cones from below in $\mathbb{R}^{n}$.

Example 2.5. If $n=1$ and $U$ is an interval, then $u \in \operatorname{CCA}(U)$ if and only if $u$ is convex. This is clear from the definition: the condition that the "secant line" joining two points on the graph of $u$ always lies above the graph is equivalent to $u \in$ CCA. Thus $\operatorname{AM}(U)$ consists of linear functions.

In fact, in any dimension and with any norm, linear functions are in $\operatorname{AM}(U)$. We leave the proof for the reader here, but it is also a special case of Example 4.2 In view of Lemma 2.12 below, the supremum of a family of linear functions will lie in CCA. Thus convex functions enjoy comparison with cones from above in any dimension and with any norm.

2.2. First Consequences. In this section we derive a simple Lipschitz continuity estimate (Lemma 2.9), which follows from a "Harnack inequality", and show that the supremum of a family of functions which enjoy comparison with cones from above also has this property (Lemma 2.12). These facts are needed for the existence proof in Section 3 The result (2.8) of the next lemma is used to derive the Lipschitz 
continuity estimate. Results in this section not just mentioned are not needed until later and may be set aside for now if one is eager to get to the existence proof.

Lemma 2.6. Let $u \in \mathrm{CCA}(U)$ and $y \in U$. Then

$$
\max _{\{w:|w-y|=r\}} u(w)=\max _{\{w:|w-y| \leq r\}} u(w) \quad \text { for } \quad 0 \leq r<\operatorname{dist}(y, \partial U) .
$$

Moreover,

$$
u(x) \leq u(y)+\max _{\{w:|w-y|=r\}}\left(\frac{u(w)-u(y)}{r}\right)|x-y|
$$

for $|x-y| \leq r<\operatorname{dist}(y, \partial U)$ and the function

$$
g^{+}(r):=\max _{\{w:|w-y| \leq r\}} u(w)=\max _{\{w:|w-y|=r\}} u(w) \text { is convex }
$$

on the interval $0 \leq r<\operatorname{dist}(y, \partial U)$.

Finally,

$$
S^{+}(y, r):=\max _{\{w:|w-y|=r\}}\left(\frac{u(w)-u(y)}{r}\right)
$$

is nonnegative and nondecreasing in $r, 0<r<\operatorname{dist}(y, \partial U)$.

Proof. Assume $u \in \mathrm{CCA}(U)$. The cone function on the right side of (2.8) bounds $u$ above on the boundary of the set $\{x: 0<|x-y|<r\}$, whence (2.8) holds as claimed. This is all that is needed from this lemma for the proof of Lemma 2.9; per the opening remarks of this section, the rest may be put aside for now. However, this is one story, so the reader may prefer to continue.

To continue, let us notice that (2.7) follows from (2.1) with $a=0$ and $V=B_{r}(y)$, and that the relation (2.7) implies that $S^{+}$in (2.10) is nonnegative. We turn to (2.9). We claim that for $0 \leq s<r<\operatorname{dist}(y, \partial U)$,

$$
u(x) \leq g^{+}(s)+\frac{g^{+}(r)-g^{+}(s)}{r-s}(|x-y|-s) \quad \text { for } \quad s \leq|x-y| \leq r .
$$

Indeed, by the definition of $g^{+}$, the cone function on the right bounds $u$ above on the boundary of the annular region $s<|x-y|<r$, whence (2.11). If

$$
\tau=\lambda s+(1-\lambda) r \quad \text { where } \quad \lambda \in[0,1],
$$

we thus have

$$
u(x) \leq g^{+}(s)+\frac{g^{+}(r)-g^{+}(s)}{r-s}(\tau-s) \quad \text { for } \quad 0 \leq|x-y| \leq \tau,
$$

as $u(x) \leq g^{+}(s)$ for $|x-y| \leq s$. Maximizing the left-hand side of (2.12) over $|x-y| \leq \tau$ yields $g^{+}(\tau)$ and, rearranging the result, (2.12) becomes

$$
g^{+}(\tau) \leq(1-\lambda) g^{+}(r)+\lambda g^{+}(s),
$$

thereby exhibiting the convexity of $g^{+}$.

For later use, we give two proofs of the monotonicity of $S^{+}$. First, from (2.8) itself it follows that if $0<|x-y| \leq r$, then

$$
\frac{u(x)-u(y)}{|x-y|} \leq \max _{\{w:|w-y|=r\}}\left(\frac{u(w)-u(y)}{r}\right)
$$


and one can maximize over $x,|x-y|=s \leq r$, to find $S^{+}(y, s) \leq S^{+}(y, r)$. Secondly, since $g^{+}$is convex, if $0<s \leq r$, in view of (2.7), (2.9) and $g^{+}(0)=u(y)$,

$$
S^{+}(y, s)=\frac{g^{+}(s)-g^{+}(0)}{s} \leq \frac{g^{+}(r)-g^{+}(0)}{r}=S^{+}(y, r) \text {. }
$$

Remark 2.7. The notation $S^{+}(y, r)$ is a mnemonic for the maximum slope of the line segments joining the point $(y, u(y))$ in the graph of $u$ with points $(w, u(w))$ where $w$ is on the sphere of radius $r$ and center $y$. By the monotonicity of $S^{+}$asserted by the lemma, this is the same as the maximum of the slopes joining $(y, u(y))$ to points in the graph over the ball with radius $r$ and center $y$. We prefer to exhibit this quantity in terms of $w$ on the sphere for later purposes.

Remark 2.8. If $u \in \mathrm{CCB}(U)$, then $-u \in \mathrm{CCA}(U)$ and applying the results of Lemma 2.6] to $-u$ yields:

$$
\begin{gathered}
\min _{\{w:|w-y|=r\}} u(w)=\min _{\{w:|w-y| \leq r\}} u(w), \\
g^{-}(r):=\min _{\{w:|w-y| \leq r\}} u(w)=\min _{\{w:|w-y|=r\}} u(w)
\end{gathered}
$$

is concave,

$$
u(x) \geq u(y)+\min _{\{w:|w-y|=r\}}\left(\frac{u(w)-u(y)}{r}\right)|x-y|
$$

for $|x-y| \leq r$, and, finally,

$$
S^{-}(y, r):=\min _{\{w:|w-y|=r\}}\left(\frac{u(w)-u(y)}{r}\right)
$$

is nonpositive and nonincreasing in $r$, all on $0<r<\operatorname{dist}(y, \partial U)$. This is a general phenomenon; all consequences of "one-sided" assumptions appearing herein immediately imply a corresponding result if these assumptions are satisfied by $-u$ in place of $u$. We often will not stop to formulate the corresponding result in what follows, leaving this to the reader.

Lemma 2.9. Let $u \in C(U)$ satisfy (2.8). If $z \in U, x, y \in B_{R}(z)$ and $R<$ $\operatorname{dist}(z, \partial U) / 4$, then

$$
|u(x)-u(y)| \leq\left(\sup _{B_{4 R}(z)} u-\sup _{B_{R}(z)} u\right) \frac{|x-y|}{R} .
$$

Proof. Assume first that $u$ is nonpositive, that is, $u \leq 0$. Let $\mathrm{d}(y)=\operatorname{dist}(y, \partial U)$, use $u(w) \leq 0$ and let $r \uparrow \mathrm{d}(y)$ in (2.8) to find

$$
\begin{aligned}
& \text { (a) } u(x) \leq u(y)\left(1-\frac{|x-y|}{\mathrm{d}(y)}\right) \text {; equivalently, } \\
& \text { (b) } u(x)-u(y) \leq-u(y)\left(\frac{|x-y|}{\mathrm{d}(y)}\right)
\end{aligned}
$$

If $z \in U$ and $4 R<\operatorname{dist}(z, \partial U)$ and $x, y \in B_{R}(z)$, then $\mathrm{d}(y) \geq 3 R$ and $|x-y| \leq 2 R$. Thus (2.17) (a) implies $u(x) \leq u(y) / 3$ for $x, y \in B_{R}(z)$ and so

$$
\sup _{B_{R}(z)} u \leq \inf _{B_{R}(z)} u / 3 \text { for } R<\operatorname{dist}(z, \partial U) / 4 \text {. }
$$


This is a "Harnack inequality".

Estimates on the modulus of continuity follow from the Harnack inequality. Assuming first that $u \leq 0$ and using (2.18) in (2.17) (b), we find

$$
\begin{aligned}
u(x)-u(y) \leq-u(y)\left(\frac{|x-y|}{\mathrm{d}(y)}\right) & \leq-\inf _{B_{R}(z)} u\left(\frac{|x-y|}{3 R}\right) \\
& \leq-\sup _{B_{R}(z)} u\left(\frac{|x-y|}{R}\right)
\end{aligned}
$$

for $x, y \in B_{R}(z), R<\mathrm{d}(z) / 4$.

If $u$ is not nonpositive, then (2.19) holds with $u$ replaced by $u-\sup _{B_{4 R}(z)} u$ (clearly if $u$ enjoys comparison with cones from above, then so does $u+$ constant). The outcome of this replacement, since $x$ and $y$ can be interchanged, is (2.16).

Remark 2.10. If one dislikes working with nonpositive functions, recall Remark 2.8 Since $u \in \operatorname{CCA}(U)$ satisfies (2.8) by Lemma [2.6] it will obey the Lipschitz estimate; likewise for $u \in \operatorname{CCB}(U)$ with appropriate changes via Remark 2.8. Finally, Lemma 2.9 is valid if $u$ is merely upper-semicontinuous, as the reader can easily see.

Remark 2.11. The estimate (2.16) implies that if $u \in \operatorname{CCA}(U)$ and $E \subset U$ is compact, then $\mathrm{L}_{u}(E)<\infty$. To see this quickly, assume otherwise. Then for each $j=1,2, \ldots$ there exists $x^{j}, y^{j}$ in $E$ such that $\left|u\left(x^{j}\right)-u\left(y^{j}\right)\right|>j\left|x^{j}-y^{j}\right|$. Since $u$ is continuous, it is bounded on $E$, and then we have $\left|x^{j}-y^{j}\right| \rightarrow 0$ as $j \uparrow \infty$. Since $E$ is compact, passing to subsequences if necessary, we can assume that $x^{j}, y^{j} \rightarrow z \in E$. However, then for $j$ large enough we can assume that $x^{j}, y^{j} \in$ $B_{R}(z)$ where $4 R<\operatorname{dist}(z, \partial U)$. The estimate (2.16) now yields a contradiction to $\left|u\left(x^{j}\right)-u\left(y^{j}\right)\right|>j\left|x^{j}-y^{j}\right|$ for large $j$.

Lemma 2.12. Let $\mathcal{F} \subset C(U)$ be a family of functions that enjoy comparison with cones from above in $U$. Suppose

$$
h(x)=\sup _{v \in \mathcal{F}} v(x)
$$

is finite and locally bounded above in $U$. Then $h \in C(U)$, and it enjoys comparison with cones from above in $U$.

Proof. First, choose $v_{0} \in \mathcal{F}$ and notice that we may replace $\mathcal{F}$ by

$$
\hat{\mathcal{F}}=\left\{\max \left(v, v_{0}\right): v \in \mathcal{F}\right\}
$$

in (2.20) without changing $h$. Moreover, $\hat{\mathcal{F}} \subset \mathrm{CCA}(U)$ (see below). We are reduced to the case in which the functions in $\mathcal{F}$ are all locally bounded above (by $h$ ) and below (by $v_{0}$ ). The continuity of $h$ now follows from Lemma 2.9, which implies that $\mathcal{F}$ is locally equicontinuous. Suppose that $V \subset \subset U$ and $z \notin V$. For $v \in \mathcal{F}$ we have, by assumption and the definition of $h$,

$$
v(x)-a|x-z| \leq \max _{w \in \partial V}(v(w)-a|w-z|) \leq \max _{w \in \partial V}(h(w)-a|w-z|)
$$

for $x \in V$. Taking the supremum over $v \in \mathcal{F}$ on the left hand side, we find that $h$ enjoys comparison with cones from above. 
2.3. Local Maxima Imply Locally Constant. This small section establishes that if $u$ satisfies a weakened variant of the consequence (2.8) of $u \in \operatorname{CCA}(U)$, then $u$ is constant in some neighborhood of a local maxima point. This observation adds a little bit of insight into the structure of absolutely minimizing functions, and it is needed in the proof of uniqueness in Section 6. In addition, (2.7), which was deduced from $u \in \mathrm{CCA}(U)$ in the course of proving Lemma 2.6 is seen to be a consequence of either (2.8) or (2.9). In Section 4 we will see that, in fact, each of (2.8) and (2.9) is equivalent to $u \in \operatorname{CCA}(U)$.

Suppose, without assuming $u \in \operatorname{CCA}(U)$, that $g^{+}$as defined in Lemma 2.6 is convex. Then, by convexity, $g^{+}(\tau)-g^{+}(0) \leq\left(g^{+}(r)-g^{+}(0)\right)(\tau / r)$ for $0 \leq \tau \leq r$. This may be restated as the weakened variant of (2.8):

$$
u(x) \leq u(y)+\max _{\{w:|w-y| \leq r\}}\left(\frac{u(w)-u(y)}{r}\right)|x-y|
$$

for $y \in U$ and $|x-y| \leq r<\operatorname{dist}(y, \partial U)$. The only difference between (2.22) and (2.8) is that the max on the right is taken over the solid ball $\{w:|w-y| \leq r\}$ instead of the sphere $\{w:|w-y|=r\}$. Thus (2.22) follows from either (2.8) or (2.9), both of which are in turn consequences of $u \in \operatorname{CCA}(U)$.

Lemma 2.13. Let $U$ be connected, $u \in C(U)$ and (2.22) hold for $x, y \in U$ with $|x-y| \leq r<\operatorname{dist}(y, \partial U)$. Let $\hat{x} \in U$ and $u(\hat{x}) \geq u(w)$ for $w \in U$. Then $u \equiv u(\hat{x})$ in $U$.

Proof. The set $\{x \in U: u(x)=u(\hat{x})\}$ is obviously closed in $U$. We show then that it is also open; since $U$ is assumed to be connected, the result follows. It suffices to show that $u$ is constant on some ball containing $\hat{x}$. If $z \in U$ and $|z-\hat{x}| \leq s<$ $\operatorname{dist}(z, \partial U)$, 2.22) with $x=\hat{x}, y=z$ and $r=s$ yields

$$
\begin{aligned}
u(\hat{x}) & \leq\left(1-\frac{|\hat{x}-z|}{s}\right) u(z)+\left(\max _{\{w:|w-z| \leq s\}} u(w)\right) \frac{|\hat{x}-z|}{s} \\
& \leq\left(1-\frac{|\hat{x}-z|}{s}\right) u(z)+u(\hat{x}) \frac{|\hat{x}-z|}{s}
\end{aligned}
$$

provided that $u(w) \leq u(\hat{x})$ for $|w-z| \leq s$. This last condition holds, by assumption, in a neighborhood of $\hat{x}$. Thus for the $z$ 's satisfying all of the above requirements, which clearly cover a neighborhood of $\hat{x}, u(z) \leq u(\hat{x}) \leq u(z)$.

Remark 2.14. Lemma 2.13 implies that if $u$ satisfies (2.22) (in particular, if $u \in$ $\mathrm{CCA}(U))$ and has a local maximum at some point $\hat{x} \in U$, then it is constant in any connected neighborhood of $\hat{x}$ for which $\hat{x}$ is a maximum point. In particular, (2.7) holds. However, $u$ need not be constant in $U$ even if $U$ is connected. The function $u(x)=\max (x, 0)$ in $\mathbb{R}$ belongs to $\operatorname{CCA}(\mathbb{R})$ (Example 2.5), but it is not constant even though -1 is a point of local maximum.

2.4. Second Consequences. In this section we derive further consequences of (2.8), which we restate here for convenience:

$$
u(x) \leq u(y)+\max _{\{w:|w-y|=r\}}\left(\frac{u(w)-u(y)}{r}\right)|x-y|
$$


for $y \in U,|x-y| \leq r<\operatorname{dist}(y, \partial U)$. It follows from the end of the proof of Lemma 2.6 and Lemma 2.13 that if (2.23) holds, then

$$
S^{+}(y, r):=\max _{\{w:|w-y|=r\}}\left(\frac{u(w)-u(y)}{r}\right)
$$

is nonnegative and nondecreasing in $r$ for $0<r<\operatorname{dist}(y, \partial U)$. The limit

$$
S^{+}(y):=\lim _{r \downarrow 0} S^{+}(y, r)=\inf _{0<r<\operatorname{dist}(y, \partial U)} S^{+}(y, r)
$$

is therefore well-defined, finite and nonnegative. We have:

Lemma 2.15. Let $u \in C(U)$ satisfy (2.23) and $y \in U$.

(i) $S^{+}(y, r)$, as given by (2.24), is nonnegative and nondecreasing in $r$, $0<r<\operatorname{dist}(y, \partial U)$.

(ii) $S^{+}(y)$, as given by (2.25), is upper-semicontinuous in $y \in U$.

(iii) If $[w, z] \subset U$, then

$$
|u(w)-u(z)| \leq\left(\max _{y \in[w, z]} S^{+}(y)\right)|z-w| .
$$

(iv) $S^{+}(y)=\mathrm{T}_{u}(y)$ where $\mathrm{T}_{u}(y)$ is defined in Section 1.5 .

(v) If $-u$ also satisfies (2.23) together with $u$, then

$$
0 \geq S^{-}(y)=\lim _{r \downarrow 0} S^{-}(y, r)=-S^{+}(y) \quad \text { for } \quad y \in U
$$

where $S^{-}(y, r)$ is defined in (2.15).

The reader will note the dual use of $S^{+}$in (2.24) and (2.25), the distinction being made only by the number of arguments displayed. We also recall here Remark 2.7

Proof. Point (i) was established in the remarks preceding the lemma. We now invoke Lemma 2.9 and Remark 2.11 to assert that if (2.23) holds, then $u$ is Lipschitz continuous on any compact subset of $U$. Let $[w, z] \subset U$. By the local Lipschitz continuity, $g(t):=u(w+t(z-w))$ is Lipschitz continuous in $t \in[0,1]$. Fix $t \in(0,1)$ and observe that (2.23) with $x=w+(t+h)(z-w)$ and $y=w+t(z-w)$ implies, for small $h>0$,

$$
\begin{aligned}
\frac{g(t+h)-g(t)}{h} & =\frac{u(w+(t+h)(z-w))-u(w+t(z-w))}{h} \\
& \leq S^{+}(w+t(z-w), h|z-w|)|z-w| .
\end{aligned}
$$

The last inequality follows from the definition of $S^{+}$. Sending $h \downarrow 0$ we find

$$
g^{\prime}(t) \leq S^{+}(w+t(z-w))|z-w| \leq\left(\sup _{y \in[w, z]} S^{+}(y)\right)|z-w|
$$

at any point of differentiability of $g$. Thus

$$
u(z)-u(w)=g(1)-g(0)=\int_{0}^{1} g^{\prime}(t) d t \leq\left(\sup _{y \in[w, z]} S^{+}(y)\right)|z-w| .
$$

Interchanging $z$ and $w$ we arrive at (2.26) with "sup" in place of "max". The upper-semicontinuity of $S^{+}$, which is proved below, allows the "sup" to be replaced by "max". 
Now

$$
S^{+}(y, r)=\max _{|w-y|=r}\left(\frac{u(w)-u(y)}{r}\right) \leq L_{u}\left(B_{r}(y)\right)
$$

and therefore $S^{+}(y) \leq \mathrm{T}_{u}(y)$. On the other hand, using (2.27) and the monotonicity of $S^{+}(y, r)$ in $r$ and the continuity in $y$, we obtain, if $s>0$ is small,

$$
\begin{aligned}
\mathrm{T}_{u}(y) & =\lim _{r \downarrow 0} L_{u}\left(B_{r}(y)\right) \leq \lim _{r \downarrow 0} \sup _{w \in B_{r}(y)} S^{+}(w) \\
& \leq \lim _{r \downarrow 0} \sup _{w \in B_{r}(y)} S^{+}(w, s)=S^{+}(y, s) .
\end{aligned}
$$

The remaining inequality, $\mathrm{T}_{u}(y) \leq S^{+}(y)$, follows upon sending $s \downarrow 0$. We have proved $S^{+}(y)=\mathrm{T}_{u}(y)$; the upper-semicontinuity of $S^{+}$follows from that of $\mathrm{T}_{u}$, which is proved in Section 1.5

The assertions regarding $S^{-}$follow from $\mathrm{T}_{(-u)}=\mathrm{T}_{u}$.

Remark 2.16. If $v \in C(U)$ and $[w, z] \subset U$, then the analog

$$
|v(w)-v(z)| \leq\left(\max _{y \in[w, z]} \mathrm{T}_{v}(y)\right)|z-w|
$$

of (2.26) holds. The proof mimics the one above.

Remark 2.17. Since $S^{+}(y, r)$ decreases to $S^{+}(y)=\mathrm{T}_{u}(y)$, it follows that

$$
\mathrm{T}_{u}(y) \leq \max _{\{w:|w-y|=r\}}\left(\frac{u(w)-u(y)}{r}\right) \leq 2 \frac{\sup _{B_{r}(y)}|u|}{r} \text { for } r<\operatorname{dist}(y, \partial U),
$$

and, if $u \leq 0$, then $\mathrm{T}_{u}(y) \leq-u(y) / r$. Letting $r \uparrow \operatorname{dist}(y, \partial U)$ then yields

$$
\mathrm{T}_{u}(y) \leq \frac{-u(y)}{\operatorname{dist}(y, \partial U)} .
$$

We also remark that $\mathrm{T}_{u}(y) \geq|D u(y)|^{*}$ if $u$ is differentiable at $y$. With this substitution in the inequality above, this is also sometimes referred to as a "Harnack inequality".

2.5. The Increasing Slope Estimate. Essential use is made of the following lemma in Sections 4 and 7

Lemma 2.18. Let $u \in C(U)$ and (2.23) hold. If $x^{0}, x^{1} \in U, 0<\left|x^{1}-x^{0}\right|<$ $\operatorname{dist}\left(x^{0}, \partial U\right)$, and

$$
u\left(x^{1}\right)-u\left(x^{0}\right)=S^{+}\left(x^{0},\left|x^{1}-x^{0}\right|\right)\left|x^{1}-x^{0}\right|,
$$

equivalently,

$$
u\left(x^{1}\right)=\max \left\{u(w):\left|w-x^{0}\right|=\left|x^{1}-x^{0}\right|\right\},
$$

then, for $0<s<\operatorname{dist}\left(x^{0}, \partial U\right)-\left|x^{1}-x^{0}\right|$,

$$
0 \leq S^{+}\left(x^{0},\left|x^{1}-x^{0}\right|\right) \leq S^{+}\left(x^{1}\right) \leq S^{+}\left(x^{1}, s\right) .
$$

Proof. The first inequality just recalls that $0 \leq S^{+}$, and the last inequality recalls the monotonicity of $S^{+}$(Lemma 2.15). What follows below will be transparent to the reader upon recalling Remark 2.7 and drawing a sketch.

First, we use, by assumption, that

$$
u(x) \leq u\left(x^{0}\right)+S^{+}\left(x^{0},\left|x^{1}-x^{0}\right|\right)\left|x-x^{0}\right| \quad \text { for } \quad\left|x-x^{0}\right| \leq\left|x^{1}-x^{0}\right| .
$$


Put

$$
x^{t}=x^{0}+t\left(x^{1}-x^{0}\right) \quad \text { for } \quad 0 \leq t \leq 1
$$

and $x=x^{t}$ in (2.31) to find, via (2.29),

$$
\begin{aligned}
u\left(x^{t}\right) & \leq u\left(x^{0}\right)+S^{+}\left(x^{0},\left|x^{1}-x^{0}\right|\right) t\left|x^{1}-x^{0}\right| \\
& =u\left(x^{0}\right)+t\left(u\left(x^{1}\right)-u\left(x^{0}\right)\right) .
\end{aligned}
$$

Thus

$$
\begin{aligned}
u\left(x^{1}\right)-u\left(x^{t}\right) & \geq(1-t)\left(u\left(x^{1}\right)-u\left(x^{0}\right)\right) \\
& =\left|x^{1}-x^{t}\right| S^{+}\left(x^{0},\left|x^{1}-x^{0}\right|\right)
\end{aligned}
$$

and therefore

$$
S^{+}\left(x^{t},\left|x^{1}-x^{t}\right|\right)=S^{+}\left(x^{t},(1-t)\left|x^{1}-x^{0}\right|\right) \geq S^{+}\left(x^{0},\left|x^{1}-x^{0}\right|\right) .
$$

Next we assume that $0<s<\operatorname{dist}\left(x^{1}, \partial U\right)$; then

$$
\left|x^{t}-x^{1}\right|<s<\operatorname{dist}\left(x^{t}, \partial U\right)
$$

if $t$ is near 1 , and then, by (2.35) and the monotonicity of $S^{+}$,

$$
S^{+}\left(x^{t}, s\right) \geq S^{+}\left(x^{t},\left|x^{1}-x^{t}\right|\right) \geq S^{+}\left(x^{0},\left|x^{1}-x^{0}\right|\right) .
$$

Letting $t \uparrow 1$ in the inequality of the extremes above yields

$$
S^{+}\left(x^{1}, s\right) \geq S^{+}\left(x^{0},\left|x^{1}-x^{0}\right|\right) .
$$

Letting $s \downarrow 0$, we arrive at (2.30).

We note for future reference, while the proof is fresh, that if we choose $x^{2}$ so that $\left|x^{2}-x^{1}\right|=s$ and $u\left(x^{2}\right)-u\left(x^{1}\right)=S^{+}\left(x^{1}, s\right)\left|x^{2}-x^{1}\right|$, then the final inequality above can be written

$$
\begin{aligned}
\frac{u\left(x^{2}\right)-u\left(x^{1}\right)}{\left|x^{2}-x^{1}\right|} & =S^{+}\left(x^{1},\left|x^{2}-x^{1}\right|\right) \\
& \geq S^{+}\left(x^{0},\left|x^{1}-x^{0}\right|\right)=\frac{u\left(x^{1}\right)-u\left(x^{0}\right)}{\left|x^{1}-x^{0}\right|} .
\end{aligned}
$$

2.6. A Sharper Harnack Inequality. Here, just for fun, we sharpen the Harnack inequality (2.18) that appeared in the course of proving Lemma 2.9. Recall that it is assumed that $u \leq 0$. Assume now that $R=\mathrm{d}(z):=\operatorname{dist}(z, \partial U)$ and $r<R$. Let $x, y \in B_{r}(z)$, let $m$ be a positive integer and define

$$
x^{j}=x+j \frac{y-x}{m} \quad \text { for } \quad j=0,1, \ldots, m .
$$

As $\left|x^{j+1}-x^{j}\right|=|x-y| / m<\mathrm{d}\left(x^{j+1}\right)$ as soon as $m$ is large and $\mathrm{d}\left(x^{j}\right) \geq R-r$, we use (2.17) (a) to assert that

$$
u\left(x^{j}\right) \leq u\left(x^{j+1}\right)\left(1-\frac{|x-y|}{m(R-r)}\right) .
$$

Iterating this relation, we conclude

$$
\begin{aligned}
u(x)=u\left(x^{0}\right) & \leq u\left(x^{m}\right)\left(1-\frac{|x-y|}{m(R-r)}\right)^{m} \\
& =u(y)\left(1-\frac{|x-y|}{m(R-r)}\right)^{m} \rightarrow u(y) e^{-\frac{|x-y|}{R-r}}
\end{aligned}
$$


or

$$
u(x) \leq u(y) e^{-\frac{|x-y|}{R-r}} \quad \text { for } \quad x, y \in B_{r}(z), 0<r<R, B_{R}(z) \subset U .
$$

In what way is this sharper than, for example, (2.18), which corresponds to $r=R / 4$ ? First of all, it is valid for all $r<R$. To compare with (2.18), we take $R=4 r$, and then (2.37) implies

$$
\sup _{B_{r}(z)} u \leq \exp (-2 / 3)\left(\inf _{B_{r}(z)} u\right)
$$

and $\exp (-2 / 3)=.5134 \ldots>1 / 3$. Again, if the reader prefers to deal with nonnegative $u \in \mathrm{CCB}$, replace $u$ by $-u$ above to find the correct inequalities.

2.7. Notes. The equivalence of comparison with cones and "absolutely minimizing" was first made explicit in Crandall, Evans and Gariepy [29] in the case of the Euclidean norm. Jensen 47 had previously shown that viscosity solutions enjoy comparison with cones by a similar method, and the full proof of this in 29] is a simplification of other arguments in [47. Crandall et al. showed that viscosity suband supersolutions of the infinity Laplace equation (0.7) may be characterized by a comparison principle with appropriate cones and demonstrated how comparison with cones directly implies the strong absolutely minimizing property (0.4). We improve and generalize their presentation of this last result in our Section 4. Subsequently, this framework was used to analyze the very subtle issue of regularity [30]. The main result of [30] is presented in Section [7] The full equivalence of comparison with cones and absolutely minimizing proved above for general norms is new. The straightforward proof of Proposition 2.1 free of viscosity solutions and differential equations, is inspired by some arguments in [5] and [49. See [26] and [40] for further generalizations.

Most of the key estimates of this section are generalizations of related arguments in [29. The role of convexity did not appear there; however L. C. Evans noticed the convexity of $g^{+}$during the preparation of [30], although no use was made of it at that time. The convexity in the case of the Euclidean norm is also noted in 17. See also the notes to Section 4. An exception is the result Lemma 2.13, which appears here for the first time in this context. Via the viscosity solution theory of the infinity Laplace operator, this point appeared for regularizations of functions enjoying comparison with cones from above in the case of the Euclidean norm in 12 in the course of proofs of their results. As mentioned before, the function $\mathrm{T}_{u}$, which plays a simplifying role in this work, appears herein for the first time.

The first version of the Harnack inequality, proved for smooth solutions of the infinity Laplace equation (0.7), is due to Evans [35]. The estimate (2.37) was obtained by Lindqvist and Manfredi in [51] with the aid of the corresponding result for the solutions of the approximating $L^{p}$ problems. It was originally stated for viscosity solutions but later extended to cover subsolutions as well [52], [48]. A simple proof of the Harnack inequality, yielding a somewhat weaker estimate, was given by Bhattacharya [16] using comparison with cones. All these results were in the case of the Euclidean norm. We obtained the sharper estimate with an elementary and straightforward argument, valid for any norm in $\mathbb{R}^{n}$. 


\section{EXISTENCE}

The existence theorem to be proved in this section is:

Theorem 3.1. Let $U$ be an open subset of $\mathbb{R}^{n}, 0 \in \partial U$, and $f \in C(\partial U)$. Let $A^{ \pm}, B^{ \pm} \in \mathbb{R}, A^{+} \geq A^{-}$, and

$$
A^{-}|x|+B^{-} \leq f(x) \leq A^{+}|x|+B^{+} \text {for } x \in \partial U .
$$

Then there exists $u \in C(\bar{U}) \cap \operatorname{AM}(U)$ such that $u=f$ on $\partial U$ and which further satisfies

$$
A^{-}|x|+B^{-} \leq u(x) \leq A^{+}|x|+B^{+} \text {for } x \in \bar{U} .
$$

The assumption $0 \in \partial U$ is not restrictive, as it can always be arranged by a translation. Alternatively, one can replace $|x|$ in (3.1) and (3.2) by $|x-z|$ where $z \in \partial U$.

In order to apply Lemma 2.12 and construct the function $u$ whose existence is asserted in Theorem 3.1 using "Perron's method", we need to find a nonempty, locally uniformly bounded family of functions enjoying comparison with cones from above and having the right kind of boundary behavior. For this purpose, we define $\underline{h}, \bar{h}: \mathbb{R}^{n} \rightarrow \mathbb{R}$ by

$$
\begin{aligned}
& \underline{h}(x)=\sup \left\{\underline{C}(x): \underline{C}(x)=a|x-z|+b, a<A^{-}, z \in \partial U, \underline{C} \leq f \text { on } \partial U\right\}, \\
& \bar{h}(x)=\inf \left\{\bar{C}(x): \bar{C}(x)=a|x-z|+b, a>A^{+}, z \in \partial U, \bar{C} \geq f \text { on } \partial U\right\} .
\end{aligned}
$$

The important properties of $\underline{h}$ and $\bar{h}$ needed in the existence proof are collected below.

Lemma 3.2. The functions $\underline{h}$ and $\bar{h}$ are well defined, continuous, and $\bar{h} \geq \underline{h}$ on $\mathbb{R}^{n}$. Moreover,

$$
A^{-}|x|+B^{-} \leq \underline{h}(x) \leq \bar{h}(x) \leq A^{+}|x|+B^{+} \text {for } x \in \mathbb{R}^{n}
$$

and

$$
\bar{h}=\underline{h}=f \text { on } \partial U \text {. }
$$

Finally, $\underline{h} \in \mathrm{CCA}(U)$ and $\bar{h} \in \mathrm{CCB}(U)$.

Proof. We first show that $\bar{h}$ is well-defined in the sense that there are functions with the properties of the $\bar{C}$ appearing in its definition. For a fixed $z \in \partial U$ and $\epsilon>0$, let $\delta>0$ be such that $f(x)<f(z)+\epsilon$ for all $x \in B_{\delta}(z) \cap \partial U$. Choose $a>\max \left\{A^{+}, 0\right\}$ so that

$$
f(z)+\epsilon+a \delta>\max _{|x-z| \leq \delta}\left(A^{+}|x|+B^{+}\right)
$$

and, if $z \neq 0$,

$$
f(z)+\epsilon+a|z|>B^{+} .
$$

Clearly, this is possible. We claim then that

$$
\bar{C}(x)=f(z)+\epsilon+a|x-z| \geq C^{+}(x):=A^{+}|x|+B^{+} \text {in } \mathbb{R}^{n} \backslash B_{\delta}(z),
$$

and therefore $\bar{C} \geq f$ on $\partial U$. To establish (3.7), notice that $a>A^{+}$implies the set

$$
W:=\left\{x \in \mathbb{R}^{n} \backslash \overline{B_{\delta}(z)}: \bar{C}(x)<C^{+}(x)\right\}
$$

is open and bounded. We claim that $W$ is empty. To see this, first notice that $\overline{B_{\delta}(z)} \cap \bar{W}=\emptyset$ by (3.5). It follows that $\bar{C}=C^{+}$on $\partial W$. As $W$ contains neither $z$ 
nor 0 (here is where we need (3.6) $), \bar{C} \equiv C^{+}$in $W$ by Section 1.4 Hence $W$ is empty. We have shown that $\bar{h}$ is well defined. We also note here that $\bar{C}(z)=f(z)+\epsilon$, and from this it follows that $\bar{h}(z)=f(z)$, so (3.4) holds for $\bar{h}$. Moreover, for $\epsilon>0$, we may clearly take $\bar{C}(x)=\left(A^{+}+\epsilon\right)|x|+B^{+}$, which implies $\bar{h} \leq C^{+}$, as in (3.3). Analogous arguments show that $\underline{h}$ also is well defined and $\underline{h} \geq A^{-}|x|+B^{-}$.

To see that $\underline{h} \leq \bar{h}$, we take any two cones $\underline{C}(x)=\underline{a}|x-\underline{z}|+\underline{b}$ and $\bar{C}(x)=\bar{a}|x-\bar{z}|+\bar{b}$ appearing in the definitions of $\underline{h}$ and $\bar{h}$, respectively. Since $\underline{C} \leq f \leq \bar{C}$ on $\partial U$, $\underline{z}, \bar{z} \in \partial U$ and $\bar{a}>A^{+} \geq A^{-}>\underline{a}$, the set where $\underline{C}>\bar{C}$ is bounded contains neither vertex and the two cones agree on the boundary. Arguing as above, we conclude that the set is empty and so $\underline{C} \leq \bar{C}$ throughout $\mathbb{R}^{n}$. This readily implies $\underline{h} \leq \bar{h}$ in $\mathbb{R}^{n}$. In particular, $\underline{h}$ and $\bar{h}$ are locally bounded, and thus the fact that $\underline{h}$ (respectively, $\bar{h}$ ) enjoys comparison with cones from above (below) follows from Lemma 2.12. Moreover, in light of Lemma 2.9, $\underline{h}$ and $\bar{h}$ are continuous in $U$.

It remains to be checked that $\underline{h}$ and $\bar{h}$ are continuous on $\partial U$. Since $\bar{h}$ is upper semicontinuous (as an infimum of continuous functions) and $\underline{h}$ is lower semicontinuous in $\mathbb{R}^{n}$, we obtain, using (3.4) and $\underline{h} \leq \bar{h}$, that

$$
f(x) \leq \liminf _{y \rightarrow x} \underline{h}(y) \leq \liminf _{y \rightarrow x} \bar{h}(y) \leq \limsup _{y \rightarrow x} \bar{h}(y) \leq f(x)
$$

for all $x \in \partial U$. This shows the continuity of $\bar{h}$, and $\underline{h}$ is treated analogously.

We need one final lemma before completing the proof of Theorem 3.1

Lemma 3.3. Suppose $u \in C(U)$ enjoys comparison with cones from above on $U$ but $u$ does not enjoy comparison with cones from below on $U$. Then there exists a nonempty set $W \subset \subset U$ and a cone function $C(x)=a|x-z|+b$ with $z \notin W$ such that $u=C$ on $\partial W, u<C$ on $W$, and the function $\hat{u}$ defined by

$$
\hat{u}=u \text { on } U \backslash W \quad \text { and } \hat{u}=C \text { on } W
$$

satisfies $\hat{u} \in \operatorname{CCA}(U)$. Moreover, if $u$ is Lipschitz continuous in $U$, then so is $\hat{u}$ and $\mathrm{L}_{\hat{u}}(U) \leq \mathrm{L}_{u}(U)$.

Proof. By Remark 2.3 there exist $W$ and $C$ satisfying the conditions of the lemma. Let $\hat{u}$ be given by 3.9 . The point here is that $\hat{u}$ then enjoys comparison with cones from above in $U$. Suppose not. Then, by Remark 2.3 there exist $\widetilde{W} \subset \subset U$ and $\widetilde{C}(x)=\tilde{b}+\tilde{a}|x-\tilde{z}|, \tilde{z} \notin \widetilde{W}$, such that $\hat{u}=\widetilde{C}$ on $\partial \widetilde{W}$ while $\hat{u}>\widetilde{C}$ in $\widetilde{W}$. As $u \in \operatorname{CCA}(U)$ and $u \leq \hat{u}=\widetilde{C}$ on $\partial \widetilde{W}$, we have $u \leq \widetilde{C}$ in $\widetilde{W}$. This implies that $\widetilde{W} \subset W$. Thus on $\partial \widetilde{W}, \widetilde{C}=\hat{u}=C$, which implies, via Section 1.4, that $\widetilde{C} \equiv C \equiv \hat{u}$ in $\widetilde{W}$. Hence $\widetilde{W}=\emptyset$, and we have reached a contradiction.

Finally, since

$$
\mathrm{L}_{\hat{u}}(W)=\mathrm{L}_{C}(\partial W)=\mathrm{L}_{u}(\partial W) \leq \mathrm{L}_{u}(U),
$$

it is easy to see that $\mathrm{L}_{\hat{u}}(U)=\max \left\{\mathrm{L}_{\hat{u}}(W), \mathrm{L}_{\hat{u}}(U \backslash W)\right\} \leq \mathrm{L}_{u}(U)$.

We complete the proof of Theorem 3.1. Define

$$
u(x):=\sup \{v(x): \underline{h} \leq v \leq \bar{h} \text { and } v \in \operatorname{CCA}(U)\} .
$$

Observe that, by Lemma 3.2 the set on the right includes $\underline{h}$, so $u$ is well defined, and, moreover, it enjoys comparison with cones from above by Lemma 2.12. By (3.4) $u \in C(\bar{U})$ and $u=f$ on $\partial U$. Thus either $u$ enjoys comparison with cones, and then $u \in \operatorname{AM}(U)$ and we are done, or $u$ does not enjoy comparison with cones from below. If $u \notin \mathrm{CCB}(U)$, Lemma 3.3 provides a larger $\hat{u}$ enjoying comparison 
with cones from above and agreeing with $u$ off a nonempty set $W \subset \subset U$ on which $\hat{u}=C>u$. Clearly $\underline{h} \leq \hat{u}$. We claim that also $\hat{u} \leq \bar{h}$ in $U$, which contradicts the definition of $u$. To see that $\hat{u} \leq \bar{h}$, assume this is false. Then the set on which $\hat{u}>\bar{h}$, call it $W^{*}$, is clearly contained in $W$, where $\hat{u}=C$. Thus $\bar{h}=C$ on $\partial W^{*}$ and, since $\bar{h}$ enjoys comparison with cones from below, $\bar{h} \geq C$ in $W^{*}$. But then $W^{*}$ is empty, a contradiction.

Remark 3.4. Let us take a closer look at the nature of Theorem 3.1. The proof really consists of two parts. The first is the construction of $\bar{h}$ and $\underline{h}$ which satisfy $\bar{h}, \underline{h} \in C(\bar{U}), \underline{h} \leq \bar{h}, \underline{h} \in \mathrm{CCA}(U), \bar{h} \in \mathrm{CCB}(U)$ and $\underline{h}=\bar{h}=f$ on $\partial U$. The second is a proof that whenever one has a pair $\underline{h}, \bar{h}$ with these properties, then $u$ given by (3.10) satisfies $u \in C(\bar{U}) \cap \mathrm{AM}(U)$ and $u=f$ on $\partial U$.

As regards the construction of $\underline{h}, \bar{h}$, one should realize that for given $f$ and $U$, various choices can be available for $A^{ \pm}$and $B^{ \pm}$. Moreover, the solution "constructed" in the proof of the theorem depends on this choice. For an extreme example, if $U=\mathbb{R}^{n} \backslash\{0\}$ and $f(0)=0$, we can take $A^{ \pm}=B^{ \pm}=0$. Then the procedure constructs $u \equiv 0$. However, we can also take $A^{ \pm}=-1, B^{ \pm}=0$, and the solution constructed is then $u(x) \equiv-|x|$. In particular, if the boundary data $f$ is bounded, then there is a bounded $u \in C(\bar{U}) \cap \operatorname{AM}(U)$ such that $u=f$ on $\partial U$ and

$$
\inf _{\partial U} f \leq u \leq \sup _{\partial U} f .
$$

This follows simply upon taking $A^{+}=A^{-}=0, B^{+}=\sup f$, and $B^{-}=\inf f$. However, if $U$ is unbounded, the example above shows that it is certainly not true that every $u \in \operatorname{AM}(U)$ with $u=f$ on $\partial U$ satisfies (3.11).

Another important case is when $f$ is Lipschitz continuous. Then we may choose as $\bar{h}$ and $\underline{h}$ the McShane-Whitney extensions of $f$ :

$$
\begin{aligned}
& \Psi(f)(x)=\inf _{y \in \partial U}\left(f(y)+\mathrm{L}_{f}(\partial U)|x-y|\right), \\
& \Lambda(f)(x)=\sup _{y \in \partial U}\left(f(y)-\mathrm{L}_{f}(\partial U)|x-y|\right),
\end{aligned}
$$

to find that

$$
u(x):=\sup \left\{v(x): \Lambda(f) \leq v \leq \Psi(f), v \in \operatorname{CCA}(U) \text { and } \mathrm{L}_{v}(U)=\mathrm{L}_{f}(\partial U)\right\}
$$

satisfies $u \in \operatorname{AM}(U), u=f$ on $\partial U$ and

$$
\mathrm{L}_{u}(U)=\mathrm{L}_{f}(\partial U) .
$$

Indeed, the constraint $\mathrm{L}_{v}(U)=\mathrm{L}_{f}(\partial U)$ is preserved both by taking a supremum and by the construction of Lemma 3.3 used above. See also Theorem 4.1 and Section 9. Again, (3.12) is not true for an arbitrary $u \in \operatorname{AM}(U)$ with $u=f$ on $\partial U$ if $U$ is unbounded. In fact, even the stringent condition $f \equiv 0$ does not guarantee the weaker property of Lipschitz continuity of such a $u$. (See Example 4.12 below and note that $u(x, y)=0$ on the line $y=x$.) On the other hand, if $U$ is bounded, then $\mathrm{L}_{u}(U)=\mathrm{L}_{u}(\partial U)$ for every $u \in \operatorname{AM}(U) \cap C(\bar{U})$, as is shown in the next section (Remark 4.8).

3.1. Notes. The first result regarding the existence of $u \in \operatorname{AM}(U)$, originally formulated in the case of a bounded open set $U$ with given Lipschitz continuous data on the boundary, also goes back to Aronsson [5. His implementation of Perron's method, appearing already in [3] and [4], can be used to produce a solution to the extension problem in a fairly abstract setting, as noted in [57], [49] and [27]. The 
more concrete variant used in this paper can be used for this purpose as well. We note again that Theorem 3.1 is considerably more general than results currently available in the literature.

Another popular approach to existence, also proposed in [5], uses an approximation procedure involving calculus of variations problems with $L^{p}$ functionals. The idea has been made precise in [18; see also [47], 64, [50], 39, 48], 19, 20], [13, 54 for further results. This approach requires much deeper prerequisites and, at first glance, seems to provide a solution to a different "absolute minimization" problem. We settle this ambiguity in the next section and discuss the approximation procedure in Section 8 .

\section{Equivalences}

In this section we show that the condition that $u$ be absolutely minimizing is equivalent to a number of other criteria besides comparison with cones. One of these replaces the requirement that for each $V \subset \subset U u$ minimizes the Lipschitz constant $\mathrm{L}_{u}(V)$ among functions which agree with $u$ on $\partial V$ (aka " $u$ is absolutely minimizing") with minimizing the functional $\sup _{x \in V}\left(\mathrm{~T}_{u}(x)\right)$ (aka " $u$ is strongly absolutely minimizing"; see Section [1.5]. This is equivalent to minimizing

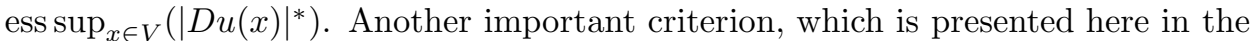
case where $|\cdot|$ is the Euclidean norm, is that $u$ should be a "viscosity solution" of the infinity Laplace equation (0.7) (the definition of which is criterion (g) of Theorem 4.1 below; see also Definition 4.10). This criterion is useful for showing that specific functions are absolutely minimizing.

4.1. Formulation of the Equivalences. Theorem 4.1 lists the equivalences mentioned above as well as a number of others. The theorem is intended to display in one place all of the kinds of equivalences of importance to us and others which merely illuminate the situation. Thus it repeats facts already proved (e.g., that $u$ is absolutely minimizing if and only if it enjoys comparison with cones). For purposes of comparison of the conditions, the statement uses the definitions rather than the names already given. Of course, providing this stand-alone panorama of equivalences comes with a loss of elegance, but it is worth the price. The various conditions are discussed after the statement. Theorem 4.1 is followed by Proposition 4.4 which consists of "one-sided" equivalences corresponding to the "two-sided" versions in the theorem. The last result formulated in this section, Proposition 4.5 implies that all the conditions considered herein are completely local; that is, if every point of a set has a neighborhood in which they hold, then they hold on the set itself.

Theorem 4.1. Let $u \in C(U)$. Then the following conditions, when imposed for every $V \subset \subset U$, are equivalent:

(a) If $L \in[0, \infty]$ and

$$
|u(w)-u(z)| \leq L|w-z| \text { for } \quad w, z \in \partial V,
$$

then

$$
|u(x)-u(y)| \leq L|x-y| \quad \text { for } \quad x, y \in V .
$$

(b) If $x \in V$, then $\Lambda\left(\left.u\right|_{\partial V}\right)(x) \leq u(x) \leq \Psi\left(\left.u\right|_{\partial V}\right)(x)$.

(c) If $a \in \mathbb{R}$ and $C(x)=a|x-z|$ where $z \notin V$, then for $x \in V$

$$
\min _{w \in \partial V}(u(w)-C(w)) \leq u(x)-C(x) \leq \max _{w \in \partial V}(u(w)-C(w)) .
$$


(d) If $v \in C(\bar{V})$ satisfies $v=u$ on $\partial V$, then

$$
\sup _{x \in V}\left(\mathrm{~T}_{u}(x)\right) \leq \sup _{x \in V}\left(\mathrm{~T}_{v}(x)\right) .
$$

The next two conditions are also equivalent to the above and do not involve the "test set" $V$ :

(e) If $y \in U$, then

(i) $g^{+}(r)=\max _{|w-y| \leq r} u(w)$ is convex for $0 \leq r<\operatorname{dist}(y, \partial U)$ and

(ii) $g^{-}(r)=\min _{|w-y| \leq r} u(w) \quad$ is concave for $\quad 0 \leq r<\operatorname{dist}(y, \partial U)$.

(f) If $y \in U$ and $|x-y| \leq r<\operatorname{dist}(y, \partial U)$, then

$$
\begin{aligned}
& \text { (i) } u(x) \leq u(y)+\max _{|w-y|=r}\left(\frac{u(w)-u(y)}{r}\right)|x-y| \text { and } \\
& \text { (ii) } u(x) \geq u(y)+\min _{|w-y|=r}\left(\frac{u(w)-u(y)}{r}\right)|x-y| \text {. }
\end{aligned}
$$

In the case that $|\cdot|$ is the Euclidean norm, the following condition is also equivalent to those above:

(g) If $\phi \in C^{2}(U)$ and $u-\phi$ has a local maximum at $\hat{x} \in U$, then

$$
\Delta_{\infty} \phi(\hat{x}):=\sum_{i, j=1}^{n} \phi_{x_{i}}(\hat{x}) \phi_{x_{j}}(\hat{x}) \phi_{x_{i} x_{j}}(\hat{x}) \geq 0 ;
$$

and if $u-\phi$ has a local minimum at $\hat{x} \in U$, then $\Delta_{\infty} \phi(\hat{x}) \leq 0$.

Remark 4.2. Condition (a) is a restatement of the definition of $u \in \operatorname{AM}(U)$. Condition (b) reintroduces the McShane-Whitney extensions, showing that they are "still around" and can be used to determine if a function is absolutely minimizing. They also reappear in an essential way in the appendix Section 9. As regards (b), the reader should recall Remark 1.2. Condition (c) is a restatement of the definition of $u \in \operatorname{CC}(U)$. Condition (d) is a restatement of the definition of $u \in \operatorname{AMS}(U)$. Conditions (e) and (f) are closely related and were derived from (c) in Lemma 2.6 (upon applying Remark 2.8). One interesting aspect of these conditions is that they follow from comparisons with cones where $V$ is the annulus between two concentric spheres or merely a punctured ball. In particular, via (f), comparison with cones on punctured balls is sufficient to imply absolutely minimizing. The formulation of (e) is elegant and supplies geometrical intuition. The viscosity solution characterization of (g) shows, in the case of the Euclidean norm, that the property of being absolutely minimizing is completely local. This is true in general via Proposition 4.5 below; however, the proof of this fact in the general case has a different flavor.

Example 4.3. We give one example of the use of (e) to verify that an important class of functions is absolutely minimizing. Suppose that $u \in C^{1}(U)$ and solves the "eikonal" equation

$$
|D u(x)|^{*}=1 \quad \text { for } \quad x \in U,
$$

where $|\cdot|^{*}$ is the norm dual to $|\cdot|$. We claim then that

$$
g^{+}(r)=\max _{|w-y| \leq r} u(w)=u(y)+r, \quad g^{-}(r)=\min _{|w-y| \leq r} u(w)=u(y)-r .
$$


Thus $u \in \operatorname{AM}(U)$ by (e). Since

$$
\begin{aligned}
u(z)-u(x) & =\int_{0}^{1} \frac{d}{d t} u(x+t(z-x)) d t \\
& =\int_{0}^{1}\langle D u(x+t(z-x)), z-x\rangle d t \\
& \leq \int_{0}^{1}|D u(x+t(z-x))|^{*}|z-x| d t=|z-x|,
\end{aligned}
$$

it is clear that $g^{+}(r)$ is Lipschitz continuous with constant 1. Fix $0 \leq r$ and let $\left|w_{r}-y\right| \leq r$ and $g^{+}(r)=u\left(w_{r}\right)$. Choose $p \in \mathbb{R}^{n}$ such that $|p|=1$ and $\left\langle D u\left(w_{r}\right), p\right\rangle=1$. Then, for $h>0$,

$$
g^{+}(r+h) \geq u\left(w_{r}+h p\right)=u\left(w_{r}\right)+h\left\langle D u\left(w_{r}\right), p\right\rangle+\mathrm{o}(h)=g^{+}(r)+h+\mathrm{o}(h) .
$$

Thus $\left(g^{+}(r+h)-g^{+}(r)\right) / h \geq 1+\mathrm{o}(1)$. Since $g^{+}(r)$ is also Lipschitz with constant 1 , we conclude that its derivative is 1 almost everywhere, verifying the first assertion of (4.2). The proof for $g^{-}$is analogous.

The simplest examples of solutions to (4.1) are nonconstant affine functions. Another important class of $C^{1}$ solutions to the eikonal equation, when $|\cdot|$ is the Euclidean norm, is provided by the distance functions to convex sets. Considering distance functions to line segments in the plane, we see that these functions are $C^{1}$ but are not necessarily $C^{2}$. Cone functions are constant multiples of the distance functions to points.

If $|\cdot|$ is not the Euclidean norm, then even cone functions are not $C^{1}$ away from their vertices. For example, if $|x|=\left|x_{1}\right|+\left|x_{2}\right|+\ldots+\left|x_{n}\right|$, then $|x|$ is not differentiable at any point of any plane $x_{j}=0$. Nonetheless, if $K \subset \mathbb{R}^{n}$ is convex, then

$$
u(x)=\operatorname{dist}(x, K) \in \operatorname{AM}\left(\mathbb{R}^{n} \backslash \bar{K}\right) .
$$

This follows from the fact that $u$ satisfies (e), the simple verification of which we leave to the reader. Note that if $u$ is Lipschitz and satisfies (4.1) almost everywhere in $\mathbb{R}^{n}$, then it need not be absolutely minimizing in $\mathbb{R}^{n}$. Cone functions are examples.

The various equivalent conditions of Theorem 4.1] with the possible exception of (d), split in a natural way into two one-sided conditions, and the corresponding onesided conditions are also equivalent. Moreover, even (d) has a one-sided analogue. Once the equivalences of the one-sided conditions are established, Theorem 4.1 follows quickly. Two-sided results are typically obtained by applying the one-sided result to $u$ and $-u$. Here are the one-sided versions:

Proposition 4.4. Let $u \in C(U)$. Then the following conditions, when imposed for every $V \subset \subset U$, are equivalent:

(a) If $L \in[0, \infty], z \in \partial V$ and

$$
u(w) \leq u(z)+L|w-z| \quad \text { for } \quad w \in \partial V
$$

then

$$
u(x) \leq u(z)+L|x-z| \text { for } \quad x \in V .
$$

(b) If $x \in V$, then $u(x) \leq \Psi\left(\left.u\right|_{\partial V}\right)(x)$. 
(c) If $a \in \mathbb{R}$ and $C(x)=a|x-z|$ where $z \notin V$, then for $x \in V$

$$
u(x)-C(x) \leq \max _{w \in \partial V}(u(w)-C(w)) .
$$

(d) If $v \in C(\bar{V})$ satisfies $v=u$ on $\partial V$ and $v \leq u$ in $V$, then

$$
\sup _{x \in V}\left(\mathrm{~T}_{u}(x)\right) \leq \sup _{x \in V}\left(\mathrm{~T}_{v}(x)\right) .
$$

The next two conditions are also equivalent to the above and do not involve the general "test set" $V$ :

(e) If $y \in U$, then

$$
g^{+}(r)=\max _{|w-y| \leq r} u(w) \quad \text { is convex for } \quad 0 \leq r<\operatorname{dist}(y, \partial U)
$$

(f) If $x, y \in U$ and $|x-y| \leq r<\operatorname{dist}(y, \partial U)$, then

$$
u(x) \leq u(y)+\max _{|w-y|=r}\left(\frac{u(w)-u(y)}{r}\right)|x-y| .
$$

In the case that $|\cdot|$ is the Euclidean norm, the following condition is also equivalent to those above:

(g) If $\phi \in C^{2}(U)$ and $u-\phi$ has a local maximum at $\hat{x} \in U$, then

$$
\Delta_{\infty} \phi(\hat{x}):=\sum_{i, j=1}^{n} \phi_{x_{i}}(\hat{x}) \phi_{x_{j}}(\hat{x}) \phi_{x_{i} x_{j}}(\hat{x}) \geq 0 .
$$

Gloriously, there is more.

Proposition 4.5. Let $u \in C(U)$. Assume that for each $x \in U$ there is a neighborhood $V \subset \subset U$ of $x$ such that $u \in \operatorname{CCA}(V)$. Then $u \in \operatorname{CCA}(U)$.

It follows that all of the conditions of Theorem 4.1 and Proposition 4.4 are completely local; they hold in $U$ iff they hold in some neighborhood of every point in $U$.

We launch into the proofs. Given the complexity of the statements, it is not surprising that the proofs will take some time. The arguments are packaged as follows. First, the discussion of the viscosity solution characterizations is deferred until Section 4.4. The following Section 4.2 treats the other equivalences asserted in Proposition 4.4 and ends with the proof of Proposition 4.5] It is followed by Section 4.3, which contains the proofs of the "nonviscosity" assertions of Theorem 4.1 In Section [5 the equation corresponding to the infinity Laplacian in the more complex situation of general norms is discussed. In Section 5 the reader will see why the viscosity solution characterization is not asserted in general (although, suitably stated, it holds for "nice" norms; see Remark 6.7).

4.2. Proof of the One-Sided Equivalences (a)-(f). Here is a road map of the sequence of implications we will establish:

$\underline{\text { The Road Map }}$

$$
\begin{aligned}
& \text { (i) } \quad(a) \Longrightarrow(b), \quad \text { (ii) } \quad(c) \Longrightarrow(a), \quad \text { (iii) }(c) \Longrightarrow(f), \\
& \text { (iv) } \quad(c) \Longrightarrow(e), \quad(\mathrm{v}) \quad(e) \Longrightarrow(f), \quad(\text { vi })(b) \Longrightarrow(c), \\
& \text { (vii) } \quad(d) \Longrightarrow(c), \quad(\text { viii })(f) \Longrightarrow(d) .
\end{aligned}
$$


We could eliminate (iii) and have a minimal set of 7 implications needed to establish the equivalence of the 6 criteria, but we would not save any work by doing so. The following implications are obvious or have already been established:

(i) (obvious), (ii) (obvious), (iii) (Lemma 2.6),

(iv) (Lemma 2.6), (v) (proof of Lemma2.6 and Lemma 2.13).

We now prove (vi): $(b) \Longrightarrow(c)$. Suppose that $(b)$ holds, but $u \notin \operatorname{CCA}(U)$. Then, by Remark 2.3, there is a nonempty set $W \subset \subset U$ and a cone function $C(x)=a|x-z|+b$ with $z \notin W$ such that $u=C$ on $\partial W$ but $u>C$ on $W$. By (b), $u \leq \Psi\left(\left.C\right|_{\partial W}\right)$ in $W$. However, by Section 1.4. $\Psi\left(\left.C\right|_{\partial W}\right)=C$, since it is an extension with the same Lipschitz constant. Thus $u \leq C$ in $W$, which is a contradiction.

We now prove (vii). To show that (d) implies (c) (equivalently, $u \in \operatorname{CCA}(U)$ ), assume that $u \notin \operatorname{CCA}(U)$. Then, by Remark 2.3 , there exists a nonempty $W \subset \subset U$ and a cone function $C(x)=a|x-y|+b$ with $y \notin W$ such that

$$
u=C \text { on } \partial W \text { and } u>C \text { in } W \text {. }
$$

By (d) with $v=C, \mathrm{~T}_{u}(x) \leq \mathrm{T}_{v}(x)=|a|$ in $W$. By Remark 2.16, we then have

$$
|u(z)-u(w)| \leq|a||z-w| \quad \text { if } \quad[w, z] \subset W .
$$

Clearly, by continuity, 4.4 continues to hold if $[w, z] \backslash\{z, w\} \subset W$. We claim that in fact (4.4) holds for all $z, w \in W$. When this is established, we are done, for then $L_{u}(W)=L_{C}(W)$ and $u=C$ on $\partial W$ implies $u=C$ in $W$ by Section 1.4 a contradiction to (4.3).

Let $z, w \in W$ and $[w, z] \backslash\{z, w\} \not \subset W$. Then there will be $y^{*}, y^{* *} \in \partial W \cap[w, z]$ such that $\left[w, y^{*}\right] \backslash\left\{y^{*}\right\} \subset W,\left[y^{* *}, z\right] \backslash\left\{y^{* *}\right\} \subset W$ and

$$
|w-z|=\left|w-y^{*}\right|+\left|y^{*}-y^{* *}\right|+\left|y^{* *}-z\right| \text {. }
$$

That is, $y^{*}\left(y^{* *}\right)$ is the first (respectively, last) point of intersection of the line segment from $w$ towards $z$ with $\partial W$. Then

$$
\begin{aligned}
|u(w)-u(z)| & \leq\left|u(w)-u\left(y^{*}\right)\right|+\left|u\left(y^{*}\right)-u\left(y^{* *}\right)\right|+\left|u\left(y^{* *}\right)-u(z)\right| \\
& \leq|a|\left|w-y^{*}\right|+\left|C\left(y^{*}\right)-C\left(y^{* *}\right)\right|+|a|\left|y^{* *}-z\right| \\
& \leq|a|\left|w-y^{*}\right|+|a|\left|y^{*}-y^{* *}\right|+|a|\left|y^{* *}-z\right|=|a||w-z| .
\end{aligned}
$$

We have used (4.4) and the comment following it on $\left[w, y^{*}\right]$ and $\left[y^{* *}, z\right]$ as well as $u=C$ on $\partial W$.

Remark 4.6. Observe that the argument above shows that if $u \in C(\bar{V})$ and

$$
|u(x)-u(y)| \leq \mathrm{L}_{u}(\partial V)|x-y|
$$

whenever $[x, y] \subset V$, then $\mathrm{L}_{u}(V)=\mathrm{L}_{u}(\partial V)$.

The remaining implication, (viii) of the road map, will use the tool provided by Proposition 4.7 below. We state the proposition, then use it to establish (viii). The generality provided by the parameter $\delta$ in the proposition plays no role in this work; it is for future reference.

Proposition 4.7. Let $U$ be bounded and $u \in C(\bar{U})$ satisfy Proposition $4.4(f)$, $x^{0} \in U, S^{+}\left(x^{0}\right)>0$ and $\delta>0$. Then there is a sequence of points $\left\{x^{j}\right\}_{j=1}^{\infty} \subset U$ and a point $x^{\infty} \in \partial U$ with the following properties:

(i) $\left|x^{j}-x^{j-1}\right| \leq \delta$ for $j=1,2, \ldots$. 
(ii) $\left[x^{j-1}, x^{j}\right] \subset U$ for $j=1,2, \ldots$.

(iii) $S^{+}\left(x^{j}\right) \geq S^{+}\left(x^{j-1}\right)$ for $j=1,2, \ldots$.

(iv) $x^{\infty}=\lim _{j \rightarrow \infty} x^{j}$.

(v) $u\left(x^{\infty}\right)-u\left(x^{0}\right) \geq S^{+}\left(x^{0}\right) \sum_{j=1}^{\infty}\left|x^{j}-x^{j-1}\right|$.

We prove (viii) of the road map, $(f) \Longrightarrow(d)$. Assume that $(f)$ holds but (d) does not. Then there are $V \subset \subset U, v \in C(\bar{V})$ and $x^{0} \in V$ for which

$$
u \geq v \quad \text { in } \quad V, \quad u=v \quad \text { on } \quad \partial V \text { and } \mathrm{T}_{u}\left(x^{0}\right)>\sup _{x \in V} \mathrm{~T}_{v}(x) .
$$

Take the point $x^{0}$ of 4.5 as the $x^{0}$ of Proposition 4.7 and take $U$ of the proposition to be the $V$ of (4.5). Recall $\mathrm{T}_{u}\left(x^{0}\right)=S^{+}\left(x^{0}\right)$ by Lemma 2.15 Let $x^{j}$ be provided by the proposition; in particular, $x^{\infty} \in \partial V$. Then, via Proposition 4.7 (v), Remark 2.16 and (4.5),

$$
\begin{aligned}
u\left(x^{\infty}\right)-u\left(x^{0}\right) & \geq S^{+}\left(x^{0}\right) \sum_{j=1}^{\infty}\left|x^{j}-x^{j-1}\right| \\
& >\sup _{V} \mathrm{~T}_{v}\left(\sum_{j=1}^{\infty}\left|x^{j}-x^{j-1}\right|\right) \geq \sum_{j=1}^{\infty}\left|v\left(x^{j}\right)-v\left(x^{j-1}\right)\right| \\
& \geq \sum_{j=1}^{\infty}\left(v\left(x^{j}\right)-v\left(x^{j-1}\right)\right)=v\left(x^{\infty}\right)-v\left(x^{0}\right) .
\end{aligned}
$$

Since $u=v$ on $\partial V, u\left(x^{\infty}\right)=v\left(x^{\infty}\right)$, and the above implies $u\left(x^{0}\right)<v\left(x^{0}\right)$, contradicting 4.5.

We proceed with the proof of Proposition 4.7 the notation and assumptions of which are now adopted. Let $x^{0} \in U$ and $S^{+}\left(x^{0}\right)>0$. Iteratively define $x^{j}$, $j=1,2, \ldots$ so that

$$
\left|x^{j}-x^{j-1}\right|=\min \left(\delta, \operatorname{dist}\left(x^{j-1}, \partial U\right) / 2\right)
$$

and

$$
\frac{u\left(x^{j}\right)-u\left(x^{j-1}\right)}{\left|x^{j}-x^{j-1}\right|}=S^{+}\left(x^{j-1},\left|x^{j}-x^{j-1}\right|\right) .
$$

By (2.30) of Lemma 2.18

$$
S^{+}\left(x^{j-1},\left|x^{j}-x^{j-1}\right|\right) \geq S^{+}\left(x^{j-2},\left|x^{j-1}-x^{j-2}\right|\right)
$$

for $j=2,3, \ldots$ Indeed, compare this statement with the redisplay (2.36) of the required part of (2.30). Thus, by the construction,

$$
\begin{aligned}
u\left(x^{j}\right)-u\left(x^{j-1}\right) & =\left|x^{j}-x^{j-1}\right| S^{+}\left(x^{j-1},\left|x^{j}-x^{j-1}\right|\right) \\
& \geq\left|x^{j}-x^{j-1}\right| S^{+}\left(x^{0},\left|x^{1}-x^{0}\right|\right) \\
& \geq\left|x^{j}-x^{j-1}\right| S^{+}\left(x^{0}\right) .
\end{aligned}
$$

These inequalities may be summed from $j=1$ to $m$ to find

$$
u\left(x^{m}\right)-u\left(x^{0}\right) \geq S^{+}\left(x^{0}\right) \sum_{j=1}^{m}\left|x^{j}-x^{j-1}\right| .
$$


Since $u$ is bounded in $\bar{U}$ and $S^{+}\left(x^{0}\right)>0$ by assumption, (4.10) implies that $\left\{x^{j}\right\}$ is Cauchy and converges to a limit $x^{j} \rightarrow x^{\infty} \in \bar{U}$; moreover, $x^{\infty} \in \partial U$ by (4.7). Passing to the limit $m \rightarrow \infty$ in (4.10) and using $u \in C(\bar{U})$, we obtain

$$
u\left(x^{\infty}\right)-u\left(x^{0}\right) \geq S^{+}\left(x^{0}\right) \sum_{j=1}^{\infty}\left|x^{j}-x^{j-1}\right| .
$$

We have now completed the road map and therefore have proved that (a)-(f) of Proposition 4.4 are equivalent.

The work of this section is completed by proving Proposition 4.5 We just sketch the proof. Under the assumptions of Proposition 4.5, if $y \in U$, the function

$$
g^{+}(r)=\max _{\{w:|w-y| \leq r\}} u(w)
$$

will be convex for $0 \leq r \leq \delta$ where $\delta$ is a (possibly small) positive number. This is because $u \in \operatorname{CCA}\left(B_{\delta}(y)\right)$ for some $\delta>0$, and we have the equivalence of (c) and (e) in Proposition 4.1. Let $R$ be the largest number satisfying $0<R \leq \operatorname{dist}(y, \partial U)$ such that $g^{+}(r)$ is convex on $[0, R)$. If $R=\operatorname{dist}(y, \partial U)$, we are done. Assuming that $R<\operatorname{dist}(y, \partial U)$, we will derive a contradiction. By compactness and the assumptions, there is some number $0<\kappa<\operatorname{dist}(y, \partial U)-R$ such that for $|w-y|=R$

$$
g_{w}^{+}(r)=\max _{\{z:|z-w| \leq r\}} u(z)
$$

is convex on $[0, \kappa]$. Then

$$
g^{+}(R+s)=\max _{\{w:|w-y|=R\}} g_{w}^{+}(s)
$$

for $0 \leq s \leq \kappa$ (we are implicitly using Lemma 2.13). As the supremum of convex functions is convex, $g^{+}(R+s)$ is convex in $s$ for $0 \leq s \leq \kappa$. One easily sees that $g^{+}(r)$ is then convex on $0 \leq r \leq R+\kappa$ if and only if the left derivative of $g^{+}(r)$ at $r=R$ is less than or equal to the right derivative of $g^{+}(r)$ at $r=R$. But if $w,|w-y|=R$, is chosen so that $g^{+}(R)=u(w)$, the right derivative of $g_{w}^{+}(s)$ at 0 , which is less than or equal to the right derivative of $g^{+}(r)$ at $r=R$ by (4.12), enjoys the desired estimate by (the proof of) Lemma 2.18 Using the equivalence of (c) and (e), we are done.

4.3. Proof of the Two-Sided Equivalences (a)-(f). The equivalence of (a), (b), (c), (e) and (f) of Theorem 4.1 follows immediately from the equivalences established for Proposition 4.4 For example, let us show that Theorem4.1(f) implies Theorem 4.1 (c). If Theorem 4.1 (f)(i) holds, then $u \in \mathrm{CCA}(U)$ by Proposition 4.4 If Theorem 4.1 (f)(ii) holds, then $-u$ satisfies condition (f)(i) in place of $u$ and thus $-u \in \mathrm{CCA}(U)$, which is the same as $u \in \operatorname{CCB}(U)$. All the two-sided conditions are implied by the one-sided conditions of Proposition 4.4 applied to both $u$ and $-u$, although a couple of minor arguments are required. To show that Proposition 4.4 (a) for $u$ and $-u$ implies Theorem 4.1 (a) for $u$, one employs the argument in the proof of Proposition 2.1. To show that Proposition 4.4 (d) for $u$ and $-u$ implies Theorem 4.1 (d), we proceed as below.

Let $u$ and $-u$ both satisfy Proposition 4.4 (d). Let $v \in C(\bar{V}), u=v$ on $\partial V$. Then by the assumptions, on the sets $V^{+}=\{x \in V: u(x)>v(x)\}$ and $V^{-}=\{x \in$ $V:-u(x)>-v(x)\}$ we have 


$$
\begin{aligned}
& \mathrm{T}_{u}(x) \leq \sup _{V^{+}} \mathrm{T}_{v} \leq \sup _{V} \mathrm{~T}_{v} \quad \text { for } \quad x \in V^{+}, \\
& \mathrm{T}_{u}(x)=\mathrm{T}_{(-u)}(x) \leq \sup _{V^{-}} \mathrm{T}_{v} \leq \sup _{V} \mathrm{~T}_{v} \quad \text { for } \quad x \in V^{-} .
\end{aligned}
$$

Thus if $\mathrm{T}_{u}\left(x^{0}\right)>\sup _{V} \mathrm{~T}_{v}$ at some point $x^{0} \in V$, it must be that $u\left(x^{0}\right)=v\left(x^{0}\right)$. Assuming this, choose a small $r>0$ and $x^{1},\left|x^{1}-x^{0}\right|=r$, such that $u\left(x^{1}\right)$ maximizes $u$ on the sphere of radius $r$ about $x^{0}$. Then, using $S^{+}\left(x^{0}\right)=\mathrm{T}_{u}\left(x^{0}\right)$,

$$
\begin{aligned}
u\left(x^{1}\right)-u\left(x^{0}\right) & =S^{+}\left(x^{0},\left|x^{1}-x^{0}\right|\right)\left|x^{1}-x^{0}\right| \\
& \geq S^{+}\left(x^{0}\right)\left|x^{1}-x^{0}\right|>\sup _{V} \mathrm{~T}_{v}\left|x^{1}-x^{0}\right| \\
& \geq v\left(x^{1}\right)-v\left(x^{0}\right)=v\left(x^{1}\right)-u\left(x^{0}\right) .
\end{aligned}
$$

Thus $x^{1} \in V^{+}$. On the other hand, using Lemma 2.18,

$$
\mathrm{T}_{u}\left(x^{1}\right)=S^{+}\left(x^{1}\right) \geq S^{+}\left(x^{0}\right)=\mathrm{T}_{u}\left(x^{0}\right)>\sup _{V} \mathrm{~T}_{v}
$$

contradicting (4.13).

Remark 4.8. Suppose $U$ is bounded, $u \in C(\bar{U}) \cap \operatorname{AM}(U)$ and $\mathrm{L}_{u}(\partial U)<\infty$. Then $\mathrm{L}_{u}(U)=\mathrm{L}_{u}(\partial U)$. Indeed, the proof of (viii) of the road map applied to both $u$ and $-u$ shows that $T_{u}(x) \leq \mathrm{L}_{u}(\partial U)$ for $x \in U$; the function $v$ in the proof of (viii) can in this case be any function for which $\mathrm{L}_{v}(U)=\mathrm{L}_{u}(\partial U)$. We combine this with Remarks 4.6 and 2.16 to verify the assertion. We recall that this claim is not true for unbounded sets $U$ by the examples of Section 1.3 .

4.4. Viscosity Solutions and the Infinity Laplace Operator in the Euclidean Norm. We begin by quickly deriving the infinity Laplace equation (which is defined below) from simple considerations of comparison with cones, independent of the concept of viscosity solutions (also defined below). Let $u: U \rightarrow \mathbb{R}$ satisfy

$$
u(x) \leq u(y)+\sup _{\{w:|w-y|=r\}}\left(\frac{u(w)-u(y)}{r}\right)|x-y|
$$

whenever $y \in U$ and $|x-y| \leq r<\operatorname{dist}(y, \partial U)$. By Proposition 4.4 this is equivalent to $u \in \operatorname{CCA}(U)$.

Suppose that $|\cdot|$ is the Euclidean norm:

$$
|x|^{2}=\sum_{j=1}^{n} x_{j}^{2} .
$$

Suppose that $u$ is twice differentiable at $y$ in the sense that there is a point $p \in \mathbb{R}^{n}$ and a symmetric $n \times n$ matrix $X$ such that

$$
u(x)=u(y)+\langle p, x-y\rangle+\frac{1}{2}\langle X(x-y), x-y\rangle+\mathrm{o}\left(|x-y|^{2}\right)
$$

as $x \rightarrow y$. We claim then that

$$
\langle X p, p\rangle \geq 0 .
$$

Just as we defined $D u$ in the pointwise sense in Section 1.5, when (4.16) holds the point $p$ and the matrix $X$ are uniquely determined and we define $D u(y):=p$, $D^{2} u(y):=X . D^{2} u$ is the Hessian of $u$. We can then rewrite (4.17) as $\Delta_{\infty} u(y) \geq 0$ via the definition

$$
\Delta_{\infty} u=\left\langle D^{2} u D u, D u\right\rangle=u_{x_{i}} u_{x_{j}} u_{x_{i} x_{j}}
$$


where the summation convention is employed.

We will prove (4.17) assuming, without loss of generality, that $y=0$ and $p \neq 0$ (4.17) is trivial if $p=0$ ). Using (4.16) in (4.14) and writing $w=r \omega$ where $|\omega|=1$, we are led to

$$
\langle p, x\rangle+\frac{1}{2}\langle X x, x\rangle+\mathrm{o}\left(|x|^{2}\right) \leq\left(\sup _{\{\omega:|\omega|=1\}}\left(\langle p, \omega\rangle+\frac{r}{2}\langle X \omega, \omega\rangle\right)+\mathrm{o}(r)\right)|x| .
$$

Dividing both sides by $|x|$, writing $\hat{x}=x /|x|$ and using $|x| \leq r$, we obtain

$$
\langle p, \hat{x}\rangle+|x| \frac{1}{2}\langle X \hat{x}, \hat{x}\rangle \leq \sup _{\{\omega:|\omega|=1\}}\left(\langle p, \omega\rangle+\frac{r}{2}\langle X \omega, \omega\rangle\right)+\mathrm{o}(r) .
$$

Letting $x \rightarrow 0$ with $\hat{x}=\hat{p}$, we find

$$
|p| \leq \sup _{\{\omega:|\omega|=1\}}\left(\langle p, \omega\rangle+\frac{r}{2}\langle X \omega, \omega\rangle\right)+o(r) .
$$

When $r=0$ the max on the right is achieved only at $\omega=\hat{p}$. Thus any maximum point $\omega_{r}$ for $r>0$ satisfies $\omega_{r} \rightarrow \hat{p}$ as $r \rightarrow 0$. Then we have

$$
\begin{aligned}
|p| & \leq\left\langle p, \omega_{r}\right\rangle+\frac{r}{2}\left\langle X \omega_{r}, \omega_{r}\right\rangle+\mathrm{o}(r) \\
& \leq|p|+\frac{r}{2}\langle X \hat{p}, \hat{p}\rangle+o(r) .
\end{aligned}
$$

It follows that $\langle X p, p\rangle \geq 0$. Similarly,

$$
u(x) \geq u(y)+\min _{\{w:|w-y|=r\}}\left(\frac{u(w)-u(y)}{r}\right)|x-y|
$$

implies that $\langle X p, p\rangle \leq 0$.

Altogether we have shown that if $u \in \operatorname{AM}(U)=\mathrm{CC}(U)$ is twice differentiable at a point $y$, then $\Delta_{\infty} u(y)=0$. For example, $C(x)=a|x-z|+b$ is absolutely minimizing in $\mathbb{R}^{n} \backslash\{z\}$, so we learn that $\Delta_{\infty} C \equiv 0$ in $\mathbb{R}^{n} \backslash\{z\}$.

Remark 4.9. In the case of the Euclidean norm, one easily sees that if $u$ is smooth, then

$$
\Delta_{\infty} u=\frac{1}{2}\left\langle D\left(|D u|^{2}\right), D u\right\rangle .
$$

This provides a proof that $C^{2}$ solutions of the eikonal equation (4.1) are absolutely minimizing, but we already know that it suffices to have $C^{1}$ regularity (Example 4.2). More generally, we see that if $u$ is $C^{2}, \Delta_{\infty} u=0$ is equivalent to $|D u|$ being constant on gradient flow lines of $u$. That is, if $\gamma$ is a curve in $\mathbb{R}^{n}$ such that

$$
\frac{d}{d t} \gamma(t)=D u(\gamma(t))
$$

then

$$
\frac{d}{d t}|D u(\gamma(t))|^{2}=2 \Delta_{\infty} u(\gamma(t))
$$

Thus, for example, the angular coordinate $\theta$ in plane polar coordinates satisfies $\Delta_{\infty} \theta=0$.

We already know that $u \in \operatorname{AM}(U)$ does not imply that $u$ is twice differentiable at every point of $U$. The distance to a line segment in the plane provides an example (see Example 4.3). We also have the example of cone functions, which are Lipschitz continuous and $C^{2}$ outside their vertices and satisfy $\Delta_{\infty} C=0$ except at the vertex, but which are not absolutely minimizing in $\mathbb{R}^{n}$. 
It is a happy and somewhat surprising fact that, nonetheless, the equation $\Delta_{\infty} u=0$, properly interpreted, characterizes the class of absolutely minimizing functions. We restate the definition of a viscosity solution here in a manner different from, but obviously equivalent to, the statement in Theorem 4.1.

Definition 4.10. A function $u \in C(U)$ satisfies $\Delta_{\infty} u \geq 0$ in $U$ in the viscosity sense if $V \subset U, v \in C^{2}(V)$ and $\Delta_{\infty} v(x)<0$ for $x \in V$, then $u-v$ does not have any local maximum points in $V$. Similarly, a function $u \in C(U)$ satisfies $\Delta_{\infty} u \leq 0$ in $U$ in the viscosity sense if $V \subset U, v \in C^{2}(V)$ and $\Delta_{\infty} v(x)>0$ for $x \in V$, then $u-v$ does not have any local minimum points in $V$. Finally, $u \in C(U)$ is a viscosity solution of $\Delta_{\infty} u=0$ in $U$ if $\Delta_{\infty} u \geq 0$ and $\Delta_{\infty} u \leq 0$ in $U$ in the viscosity sense.

Example 4.11. Let $u \in C^{2}(U)$ satisfy $\Delta_{\infty} u=0$ in $U$. Then $u$ is a viscosity solution of $\Delta_{\infty} u=0$ in $U$. To see this, suppose $V \subset U, v \in C^{2}(V), \Delta_{\infty} v<0$ in $V$. If $D v(x)=D u(x)$ and $D^{2} u(x) \leq D^{2} v(x)$ (as must be the case if $u-v$ has a local maximum point at $x$ ), it would follow that

$$
\begin{aligned}
0>\Delta_{\infty} v(x) & =\left\langle D^{2} v(x) D v(x), D v(x)\right\rangle \\
& =\left\langle D^{2} v(x) D u(x), D u(x)\right\rangle \\
& \geq\left\langle D^{2} u(x) D u(x), D u(x)\right\rangle \\
& =\Delta_{\infty} u(x)=0,
\end{aligned}
$$

a contradiction. Hence $u-v$ cannot have a local maximum. Thus $\Delta_{\infty} u \geq 0$ in the viscosity sense. The "other side" is analogous.

Conversely, if $u \in C^{2}(U)$ satisfies $\Delta_{\infty} u \geq 0$ in $U$ in the viscosity sense, then $\Delta_{\infty} u(x) \geq 0$ for every $x \in U$. This follows immediately from the definition as every point is a maximum point for $0=u-u$.

Example 4.12. We claim that $u(x, y):=x^{4 / 3}-y^{4 / 3}$ is a viscosity solution of $\Delta_{\infty} u=0$ in $\mathbb{R}^{2}$. (As one of the authors objected to this notation, we mention that we regard $x^{4 / 3}=\left(x^{4}\right)^{1 / 3}$ as well-defined for $x \in \mathbb{R}$.) This is an important example in the subject as it is, as of the moment, the least regular absolutely minimizing function known in the case of the Euclidean norm. Up to this point, in the case of the Euclidean norm, the least regular absolutely minimizing functions that have been exhibited are the distance functions to convex sets. These functions have locally Lipschitz continuous first derivatives. The current example has first derivatives which are merely Hölder continuous with exponent $1 / 3$.

A calculation verifies $\Delta_{\infty} u=0$ off the axes $x=0$ and $y=0$. However, $u$ is not twice differentiable on the axes. Hence, if $v$ is twice continuously differentiable and $\Delta_{\infty} v<0$, then any local maximum $\left(x_{0}, y_{0}\right)$ of $u(x, y)-v(x, y)$ must be of the form $\left(0, y_{0}\right)$ or $\left(x_{0}, 0\right)$. Assume, to illustrate, that $u-v$ has a maximum at $(0,1)$. Then

$$
u(x, 1)-v(x, 1)=x^{4 / 3}-1-v(x, 1) \leq u(0,1)-v(0,1)=-1-v(0,1)
$$

for $x$ near 0 . Thus $x^{4 / 3} \leq v(x, 1)-v(0,1)$, which cannot hold for a twice continuously differentiable function $v$ as $x \rightarrow 0$.

The argument above did not use that $\Delta_{\infty} v<0$. We do need this to rule out $(1,0)$ as a local maximum point of $u-v$. If $(1,0)$ is a maximum point, then $v_{y}(1,0)=$ $u_{y}(1,0)=0$ and therefore $\Delta_{\infty} v(1,0)=v_{x}^{2}(1,0) v_{x x}(1,0)$. Hence $v_{x x}(1,0)<0$. But this contradicts

$$
x^{4 / 3}-v(x, 0) \leq 1^{4 / 3}-v(1,0)
$$

for $x$ near 1 , as this implies $v_{x x}(1,0) \geq(4 / 3)(1 / 3)$. 
In view of these examples and the next result, $C^{2}$ solutions of $\Delta_{\infty} u=0$ and $(x, y) \mapsto x^{4 / 3}-y^{4 / 3}$ are absolutely minimizing.

Theorem 4.13. Let $u \in C(U)$. Then $u$ is absolutely minimizing in $U$ if and only if $u$ is a viscosity solution of $\Delta_{\infty} u=0$ in $U$.

Proof. We will in fact show that $u \in \operatorname{CCA}(U)$ iff $\Delta_{\infty} u \geq 0$ in $U$ in the viscosity sense. The theorem then follows upon applying this result to $-u$ to obtain "the other side".

Assume first that $\Delta_{\infty} u \geq 0$ in $U$ in the viscosity sense. We claim then that $u \in \operatorname{CCA}(U)$. If not, by Remark 2.3. there is a $V \subset \subset U$ and a cone function $C(x)=a|x-z|+b$ with $z \notin V$ such that $u=C$ on $\partial V$ and $u>C$ in $V$. Adding a small constant to $C$, we can assume $u<C$ on $\partial V$ and still have $\{x \in V: u(x)>$ $C(x)\} \neq \emptyset$. Thus we have

$$
u<C \text { on } \partial V, \quad\{x \in V: u(x)>C(x)\} \neq \emptyset .
$$

If for each $\epsilon>0$ we can find a perturbation $P \in C^{2}(\bar{V})$ such that $|P| \leq \epsilon$ in $V$ and

$$
\Delta_{\infty}(C+P) \leq-\delta<0 \text { in } V
$$

we will be done. Indeed, since $|P| \leq \epsilon$, (4.24) implies that the maximum of the function $u-(C+P)$ over $\bar{V}$ is attained in $V$ if $\epsilon$ is sufficiently small. But in view of $\Delta_{\infty}(C+P)<0$ in $V$, this contradicts the assumption that $u$ is a viscosity solution of $\Delta_{\infty} u \geq 0$.

Suitable perturbations can be explicitly constructed. Put $P=-\gamma|x-z|^{2}$ and $\gamma>0$. Then $C(x)+P(x)=G(|x-z|)$ where $G(s)=a s-\gamma s^{2}+b$. A direct computation shows that

$$
\Delta_{\infty} G(|x-z|)=G^{\prime \prime}(|x-z|) G^{\prime}(|x-z|)^{2},
$$

so $\Delta_{\infty}(C+P)(x)=-2 \gamma(2 \gamma|x-z|-a)^{2}$. This is strictly negative in $V$ if either $a \leq 0$ or if $a>0$ and $0<\gamma$ is sufficiently small. If $\gamma$ is sufficiently small, we also attain $|P| \leq \epsilon$ in $V$.

It remains to see that $u \in \operatorname{CCA}(U)$ implies that $\Delta_{\infty} u \geq 0$ in the viscosity sense. Assume not, so that there are $V \subset \subset U$ and $v \in C^{2}(V)$ such that $\Delta_{\infty} v<0$ in $V$ and $u-v$ has a local maximum point in $V$. Without loss of generality, we may assume that this point is 0 . Then $0 \in V$ and for some $r>0$,

$$
u(x)-v(x) \leq u(0)-v(0) \text { for }|x|<r .
$$

We seek $z \in \mathbb{R}^{n} \backslash\{0\}$ and $a \geq 0$ such that $C(x)=a|x-z|$ satisfies

$$
D C(0)=D v(0) \text { and } D^{2} C(0)>D^{2} v(0) .
$$

Let us assume for a moment that $C$ does satisfy 4.26). Elementary calculus then implies that $v-C$ has a strict maximum point at 0 , for the gradient of $v-C$ vanishes at 0 and the Hessian is strictly negative. That is,

$$
v(x)-C(x)<v(0)-C(0) \text { for small }|x| \neq 0 .
$$

Using this in conjunction with (4.25), we obtain

$$
u(x) \leq v(x)-v(0)+u(0)<C(x)-C(0)+u(0) \text { for small }|x| \neq 0 .
$$

The point is that $u(x)<\tilde{C}(x):=C(x)-C(0)+u(0)$ on $|x|=s$ if $s>0$ is sufficiently small, but $u(0)=\tilde{C}(0)$, contradicting $u \in \operatorname{CCA}(U)$. 
It remains to show that $C(x)=a|x-z|$ satisfying (4.26) can be constructed. We compute

$$
D C(0)=-a \frac{z}{|z|}, \quad D^{2} C(0)=\frac{a}{|z|}(I-\hat{z} \otimes \hat{z}),
$$

where for any nonzero vector $\hat{z}=z /|z|$ is the direction of $z$ and $\hat{z} \otimes \hat{z}$ is the projection along $\hat{z}$. Thus $D C(0)=D v(0)$ amounts to

$$
a=|D v(0)| \text { and } \hat{z}=-\widehat{D v(0)} \text {. }
$$

Here we recall that

$$
\Delta_{\infty} v(0)=\left\langle D^{2} v(0) D v(0), D v(0)\right\rangle<0
$$

so $D v(0) \neq 0$. Using the information already determined about $C$, the remaining condition $D^{2} C(0)>D^{2} v(0)$ amounts to

$$
\frac{|D v(0)|}{|z|}(I-\widehat{D v(0)} \otimes \widehat{D v(0)})>D^{2} v(0) \text {. }
$$

The problem has been reduced to showing that if $|z|$ is sufficiently small, then (4.28) holds. We leave to the reader the linear algebra exercise of showing that (4.27), which is clearly necessary in order that (4.28) hold, is also sufficient to guarantee (4.28) holds if $|z|$ is sufficiently small. A proof may also be found in [33, from which this presentation is taken.

4.5. Notes. The proof of the equivalence between absolute minimizers and strong absolute minimizers is to a large extent an adaptation of the original arguments in 29 to the current setting. Here we separated out the one-sided implications of Proposition 4.4, which did not appear in 29, but do rely on the ideas of proofs in [29]. The proof of the more difficult one of the two implications, that $\operatorname{AM}(U) \subset$ $\operatorname{AMS}(U)$, is in spirit a discrete version of the reasoning used by Aronsson in [5], Theorem 7 .

The characterization using the convexity of the maximum over balls as a function of the radius is presented here for the first time. However, beforehand, L. Caffarelli remarked (in a personal communication) that, in the Euclidean case, this property characterizes viscosity solutions of $\Delta_{\infty} u \geq 0$.

The other equivalences of this section appear here for the first time; this includes the "local implies global" result in the absence of a viscosity solution formulation. The one-sided version of condition (d) in Theorem 4.1 is the analogue of the concept of "subextremal" or "subminimum" appearing in the classical calculus of variations; see, e.g., 43, and 42.

There is an equivalence which we have not yet discussed. Roughly speaking, one can replace " $L|z-w|$ " and " $L|x-y|$ " in Theorem 4.1 (a) by $d_{V}(z, w)$ and $d_{V}(x, y)$ where $d_{V}$ is the distance between points measured using paths in $V$. It would take time to explain the precise definitions, and the one-sided analogue is, at present, a bit unsettled. Hence we have not included this alternative formulation. It is also the case that if $U$ is bounded and $u \in C(\bar{U}) \cap \operatorname{AM}(U)$, then $\sup _{U} \mathrm{~T}_{u}(x)$ is the least Lipschitz constant for $\left.u\right|_{\partial U}$ with respect to $d_{U}$.

The infinity Laplace equation (4.18) made its first public appearance in [5]. It was originally derived as a formal limit, as $p \rightarrow \infty$, of the Euler equations of the approximating $L^{p}$ problems, called $p$-Laplace equations, which explains the name it has been given. See Section 8 . The proof that the Euler equation (4.18) can be obtained directly from the comparison with cones property is new. While 
this derivation did not, as presented, establish the equivalence between viscosity solutions of the infinity Laplace equation and the class of functions enjoying comparison with cones, use of the tools in Section [6] allows this to be easily done. See Remark 6.7

Regarding Theorem 4.13 Aronsson [5] showed that $u \in C^{2}(U)$ is absolutely minimizing if and only if it satisfies the infinity Laplace equation. Viscosity solutions were first used in connection with the infinity Laplace operator in 18. The validity of the full statement of Theorem 4.13 is due to Jensen [47], although he did use a different definition for the absolute minimizers. The proof presented above is taken from [29], as modified in [33], and, unlike Jensen's proof, it is independent of the uniqueness results.

Our examples of absolutely minimizing functions so far are those explained in Example 4.3 and Example 4.12 In the Euclidean case, these are due to Aronsson. Example 4.12] belongs to a larger class of "singular solutions" constructed in [8] and 9]. The solutions constructed by Aronsson include separated solutions of the form $r^{k} f(\theta)$ in the plane, where $r, \theta$ are polar coordinates. A nice case found in [9] is $u=r^{k} \exp \left(\theta \sqrt{k-k^{2}}\right)$ for $0<k<1$. Example 4.12 is especially interesting, as it shows that a variety of results for classical solutions to the infinity Laplace equation are just false for viscosity solutions (and hence for absolutely minimizing functions). The reader may consider the behavior of $|D u|$ on the curves of gradient flow for this example as an instance. Moreover, Aronsson [6] showed that the gradient of nonconstant classical solutions of the infinity Laplace equation will never vanish and global classical solutions of this equation are affine (both results being in $\mathbb{R}^{2}$ ). Example 4.12 shows that both of these properties fail for general viscosity solutions.

\section{The INFINITy LAPLACE OPERATOR IN GENERAL NORMS}

In Section 4.4 it was assumed that $|\cdot|$ is the Euclidean norm. One may ask which of the results persist in the case of a general norm $|\cdot|$. In this section we explain how to derive, in the case of a general norm, the conditions corresponding to $\Delta_{\infty} u=0$ in the Euclidean case. Details are provided in Example 5.3 for the case of the " $\ell q$ " norm on $\mathbb{R}^{n}$. However, we stop short of rerunning the analysis of the equivalence between viscosity solutions of the necessary conditions found and comparison with cones, leaving this to the reader. In the language below, this is true if $J$ is single-valued. See Remark 6.7 for a different look at the question.

We first note that the arguments leading to 4.22 can be rerun with the following modifications. Given $y \in \mathbb{R}^{n}$, we define

$$
J(y)=\left\{x \in \mathbb{R}^{n}:|x| \leq 1 \text { and }\langle y, x\rangle=|y|^{*}\right\} .
$$

Here we use the dual norm $|\cdot|^{*}$ defined in Section 1.1 We call $J$ the "duality map" (from $\left(\mathbb{R}^{n},|\cdot|^{*}\right)$ to $\left.\left(\mathbb{R}^{n},|\cdot|\right)\right) 2$ It is easy to see that $J(y)$ is always a nonempty, closed and convex set. For example, $J(0)=\overline{B_{1}(0)}$ no matter what the norm is, while if $|\cdot|$ is the Euclidean norm and $y \neq 0$, then $J(y)=\{y /|y|\}$. In general, if $y \neq 0$, then $|x|=1$ if $x \in J(y)$. If, as in the Euclidean case, $J(y)$ is "single-valued" for $y \neq 0$, that is, it is a singleton, we do not distinguish between the set $J(y)$ and its element.

${ }^{2}$ If you have met duality maps before, please refer to the notes to this section. 
Repeating the derivation of (4.22), but now requiring that $\hat{x} \in J(p)$ as $x \rightarrow 0$, yields

$$
\begin{aligned}
|p|^{*} & \leq\left\langle p, \omega_{r}\right\rangle+\frac{r}{2}\left\langle X \omega_{r}, \omega_{r}\right\rangle+\mathrm{o}(r) \\
& \leq|p|^{*}+\frac{r}{2}\left\langle X \omega_{r}, \omega_{r}\right\rangle+\mathrm{o}(r),
\end{aligned}
$$

where $\omega_{r}$ is the unit vector maximizing the same expression as before. This time all we can say about $\omega_{r}$ is that all its limit points as $r \downarrow 0$ lie in $J(p)$. In this way, at a point $x$ of twice differentiability of either (a) $u \in \operatorname{CCA}(U)$ or (b) $v \in \mathrm{CCB}(U)$, we find, respectively,

$$
\text { (a) } 0 \leq \max _{\omega \in J(D u(x))}\left\langle D^{2} u(x) \omega, \omega\right\rangle \quad \text { or } \quad \text { (b) } \min _{\omega \in J(D v(x))}\left\langle D^{2} v(x) \omega, \omega\right\rangle \leq 0 .
$$

In combination, if $u$ is absolutely minimizing and twice differentiable at a point $x$, then

$$
\min _{\omega \in J(D u(x))}\left\langle D^{2} u(x) \omega, \omega\right\rangle \leq 0 \leq \max _{\omega \in J(D u(x))}\left\langle D^{2} u(x) \omega, \omega\right\rangle .
$$

In the event that the duality map is single-valued and $u \in \mathrm{CC}(U)$, at points of twice differentiability of $u$ where $D u \neq 0$, we therefore have the analogue

$$
\left\langle D^{2} u J(D u), J(D u)\right\rangle=0
$$

of $\Delta_{\infty} u=0$. When $D u=0$, this equation still holds if it is interpreted to mean that $X$ has both a nonnegative and a nonpositive eigenvalue.

Remark 5.1. We have scaled things differently in this section. In the Euclidean case, $J(D u)=D u /|D u|$ is the unit vector in the direction of $D u$ when $D u \neq 0$ and $J(0)$ is the closed unit ball. Compared with the previous case, we have obtained additional information when $D u=0$. Note that with this scaling, $J(\lambda p)=J(p)$ for $\lambda>0$.

Example 5.2. The equivocal nature of (5.4) is not an artifact of the derivation. For example, the function $u(x, y)=x^{2}-y^{2}$ in $\mathbb{R}^{2}$ is absolutely minimizing for the norm $|(x, y)|_{\infty}=\max (|x|,|y|)$. This can be seen by using Theorem 4.1 (e) and computations. However, using the computation of $J$ in Example 5.3 below, it is also easily seen that the "max" and "min" in (5.4) are necessary; it is not true that $\left\langle D^{2} u(x, y) \omega, \omega\right\rangle=0$ for every $\omega \in J(D u(x, y))$ when it is not single-valued and $D u(x, y) \neq 0$.

Example 5.3. Let $|\cdot|=|\cdot|_{q}$, where

$$
|x|_{q}:=\left(\sum_{i=1}^{n}\left|x_{i}\right|^{q}\right)^{1 / q}, \quad \text { if } 1 \leq q<\infty,
$$

and

$$
|x|_{\infty}:=\max \left\{\left|x_{1}\right|,\left|x_{2}\right|, \ldots,\left|x_{n}\right|\right\} .
$$

We will refer to $|\cdot|_{q}$ as the $\ell^{q}$-norm in $\mathbb{R}^{n}$. For this family of norms, the duality relations and the corresponding duality maps are easily found. First, if $1<q<\infty$, then $|\cdot|_{q}^{*}=|\cdot|_{q^{*}}$, where $q^{*}=\frac{q}{q-1}$ is the Hölder conjugate of $q, \frac{1}{q}+\frac{1}{q^{*}}=1$. To see this, note first that Hölder's inequality

$$
\langle x, y\rangle=\sum_{i=1}^{n} x_{i} y_{i} \leq\left(\sum_{i=1}^{n}\left|x_{i}\right|^{q}\right)^{1 / q}\left(\sum_{i=1}^{n}\left|y_{i}\right|^{q^{*}}\right)^{1 / q^{*}}
$$


implies

$$
|y|_{q}^{*}=\max \left\{\langle x, y\rangle: x \in \mathbb{R}^{n},|x|_{q}=1\right\} \leq\left(\sum_{i=1}^{n}\left|y_{i}\right|^{q^{*}}\right)^{1 / q^{*}}=|y|_{q^{*}} .
$$

On the other hand, if we define $\bar{x}$ by setting

$$
\bar{x}_{i}=|y|_{q^{*}}^{-q^{*} / q}\left|y_{i}\right|^{q^{*}-2} y_{i}, \quad i=1, \ldots, n,
$$

then $|\bar{x}|_{q}=1$ and

$$
\langle\bar{x}, y\rangle=|y|_{q^{*}}^{-q^{*} / q} \sum_{i=1}^{n}\left|y_{i}\right|^{q^{*}}=|y|_{q^{*}}
$$

In this case $J$ is single-valued, and it is given by

$$
J(y)=|y|_{q^{*}}^{-q^{*} / q}\left(\left|y_{1}\right|^{q^{*}-2} y_{1}, \ldots,\left|y_{n}\right|^{q^{*}-2} y_{n}\right)
$$

for $y \neq 0$.

As expected, the cases $q=1$ and $q=\infty$ require some additional care. If $|\cdot|=|\cdot|_{\infty}$, then it is not hard to see that $|\cdot|_{\infty}^{*}=|\cdot|_{1}$. The relation

$$
\langle y, x\rangle=y_{1} x_{1}+\cdots+y_{n} x_{n}=|y|_{1}=\left|y_{1}\right|+\left|y_{2}\right|+\cdots+\left|y_{n}\right|
$$

coupled with $|x|_{\infty}=1$ determines $x_{j}=y_{j} /\left|y_{j}\right|=: \operatorname{sign}\left(y_{j}\right)$ when $y_{j} \neq 0$. However, if $y_{j}=0$, then $x_{j}$ can be any number in the interval $[-1,1]$. We define $\operatorname{sign}(0)=$ $[-1,1]$. Thus, in this case,

$$
J(y)=\left\{x: x_{j} \in \operatorname{sign}\left(y_{j}\right) \text { for } j=1,2, \ldots, n\right\},
$$

where we identify $\operatorname{sign}\left(y_{j}\right)$ and the singleton set with the element $\operatorname{sign}\left(y_{j}\right)$ when $y_{j} \neq 0$.

On the other hand, if we take $|\cdot|=|\cdot|_{1}$, then $|\cdot|_{1}^{*}=|\cdot|_{\infty}$. Indeed, in general $\left(|\cdot|^{*}\right)^{*}=|\cdot|$. If $y \neq 0$, the relation

$$
\langle y, x\rangle=y_{1} x_{1}+\cdots+y_{n} x_{n}=|y|_{\infty}
$$

coupled with $|x|_{1}=1$ implies $x_{j}=0$ if $\left|y_{j}\right| \neq|y|_{\infty}$, and $x_{j}=\alpha_{j} \operatorname{sign}\left(y_{j}\right)$ if $\left|y_{j}\right|=$ $|y|_{\infty}$ where $0 \leq \alpha_{j} \leq 1$ and

$$
\sum_{j \in I(y)} \alpha_{j}=1 \quad \text { where } \quad I(y)=\left\{j:\left|y_{j}\right|=|y|_{\infty}\right\} .
$$

Thus, in this case, if $y \neq 0$, then

$$
J(y)=\left\{x:|x|_{1}=1, x_{j}=0 \text { if } j \notin I(y) \text { and } \operatorname{sign}\left(x_{j}\right)=\operatorname{sign}\left(y_{j}\right) \text { if } j \in I(y)\right\} .
$$

Cones associated to $\ell^{q}$ norms for $q=1, \frac{4}{3}, 2,4$ and $q=\infty$ are illustrated in Figure 5.1. Observe that for $q=1$ and $q=\infty$ the cones are not smooth.

Remark 5.4. After seeing Example 5.3. one might be curious to know if there is a " $q$-analogue" for every $1<q<\infty$ of the Euclidean absolute minimizer $u(x, y)=$ $x^{4 / 3}-y^{4 / 3}$ displayed in Example 4.12. It turns out that the function

$$
v_{q}(x, y)=|x|^{\alpha(q)}-|y|^{\alpha(q)}, \quad \alpha(q)=\frac{2 q}{q+1},
$$

is indeed an absolute minimizer for the norm $|\cdot|_{q}$, and $v_{2}=u$ as it should be. Moreover, the formal limit as $q \rightarrow \infty$ of the $v_{q}$ 's is $v_{\infty}(x, y)=x^{2}-y^{2}$, which was seen to be an absolute minimizer for the norm $|\cdot|_{\infty}$ in Example 5.2 . 

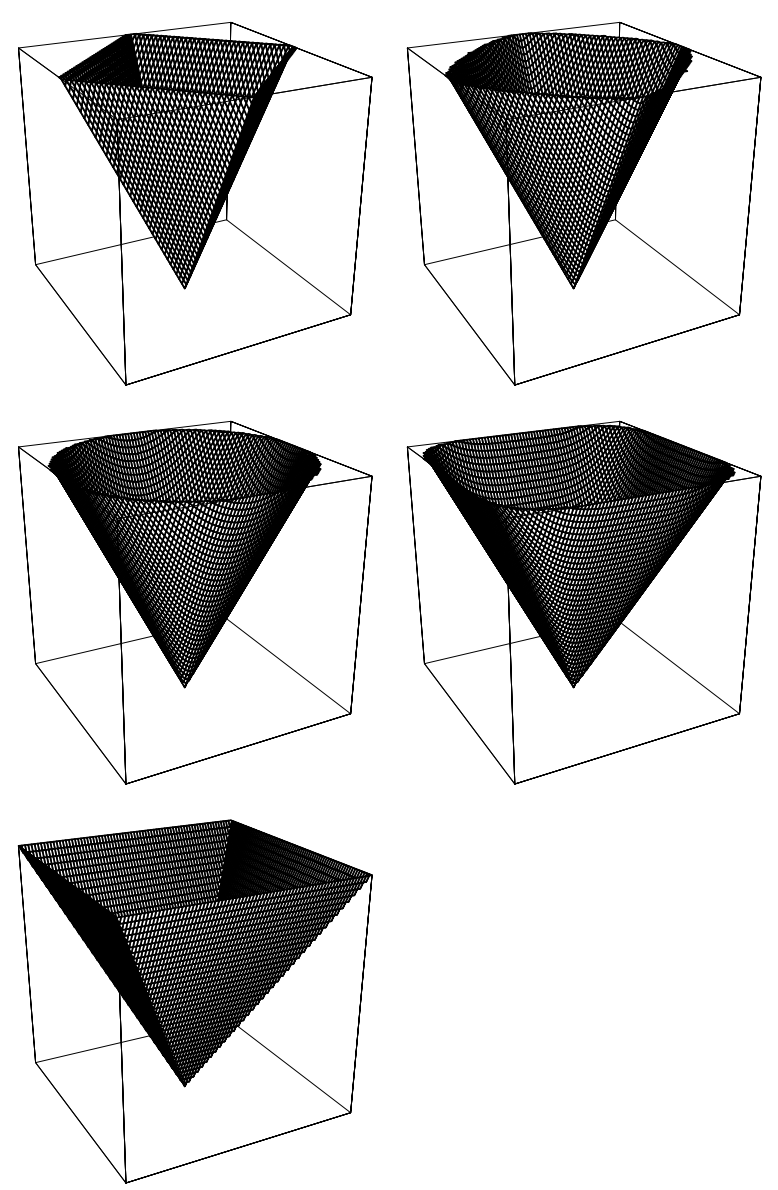

FiguRE 5.1. $\ell^{q}$-cones, $q=1, \frac{4}{3}, 2,4, \infty$.

Remark 5.5. One way to verify that the duality map $J$ is single-valued is to check that the norm $|\cdot|$ is strictly convex. In other words, the unit sphere $\{x:|x|=1\}$ contains no nontrivial line segments. Indeed, if $J(y)$ is not a singleton for some $y \neq 0$, then the unit sphere $\{x:|x|=1\}$ necessarily contains a line segment. This follows because $J(y)$ is convex and $J(y) \subset\{x:|x|=1\}$ for $y \neq 0$. Conversely, if $|x|=|y|=1,|x / 2+y / 2|=1$, and $z \neq 0$ and

$$
\left\langle\frac{x}{2}+\frac{y}{2}, z\right\rangle=|z|^{*},
$$

then, clearly, $\langle x, z\rangle=\langle y, z\rangle=|z|^{*}$ and $[x, y] \subset J(z)$. Thus $J$ is single-valued if and only if $|\cdot|$ is strictly convex.

5.1. Notes. We mention that our definition of the "duality map" $J$ (from ( $\mathbb{R}^{n}$, $\left.|\cdot|^{*}\right)$ to $\left.\left(\mathbb{R}^{n},|\cdot|\right)\right)$ uses a "normalization" at variance with the most common one. See, e.g., Reich [58].

The infinity Laplace equation and related problems for norms other than the standard Euclidean one have been considered at least in [15] and [44. However, 
these papers were primarily concerned with other issues and focused on particular norms. Hence they did not treat the issues of this section in full generality. Related equations, which go outside of the framework of this manuscript, are found in [13], [22], [19], [28], 62] and [65]. Example 5.2 is new.

\section{The Uniqueness Question}

In this section we first assume that $|\cdot|$ is the Euclidean norm. However, after studying the proof of the main result, Theorem 6.1 below, the reader can confirm that it remains true if the duality map $J$ is single-valued. The changes needed to establish this are very minor, but on a first pass through the arguments we prefer to avoid the additional notation needed for the general case. See Remark 6.6.

Theorem 6.1. Let $U$ be bounded, $|\cdot|$ be the Euclidean norm, $u \in \operatorname{CCA}(U) \cap C(\bar{U})$, $v \in \mathrm{CCB}(U) \cap C(\bar{U})$ and $u \leq v$ on $\partial U$. Then $u \leq v$ in $U$.

Remark 6.2. In particular, if $u, v \in \operatorname{AM}(U) \cap C(\bar{U})=\mathrm{CC}(U) \cap C(\bar{U})$ agree on $\partial U$, then $u=v$. In other words, when $|\cdot|$ is the Euclidean norm and $U$ is bounded, the solution of (BVP) (see Section 1.3) provided by Theorem 3.1 is unique. The result also shows that if $u, v$ are solutions of (BVP) with boundary values $f, g$, respectively, and $f \leq g$, then $u \leq v$ in $U$. These corollaries, and thus also Theorem 6.1, fail in general if $U$ is unbounded; see Section 1.3.

The proof is surprisingly subtle and complex. Some standard tools of viscosity solution theory are used in the proof. However, we give an (almost) self-contained presentation which does not refer to this theory. The tools employed are interesting and some of them are elegant, so it seems to us to be useful to present them clearly in this setting. The discussion is therefore considerably longer than it would be if these tools were taken as known, but the utility of having a self-contained presentation of this rich collection of ideas using the simplifications available in our situation seems self-evident. In particular, a reader not versed in partial differential equations can follow the flow of ideas here and learn, in this clear but nontrivial example, something about the workings of viscosity solution theory.

To orient the reader, we present a preview of what is to follow. All known proofs of Theorem 6.1 rely on the fact that $u \in \operatorname{CCA}(U)$ and $v \in \operatorname{CCB}(U)$ satisfy the inequalities derived in Section 4.4 at points of second differentiability; in particular

$$
\Delta_{\infty} v(x) \leq 0 \leq \Delta_{\infty} u(x)
$$

whenever $x$ is a point at which both $u$ and $v$ have two derivatives in the pointwise sense. Let us for a while assume that $u, v \in C^{2}(U) \cap C(\bar{U})$, (6.1) holds and $u \leq v$ on $\partial U$. First note that if we can prove $u \leq v$ in $U$ under the stronger assumption $u-v \leq-\gamma<0$ on $\partial V$ where $\gamma>0$, then we are done. Indeed, under the assumptions of the theorem, $u-\gamma \in \operatorname{CCA}(U) \cap C(\bar{U})$, and $\tilde{u}=u-\gamma$ satisfies $\tilde{u}-v \leq-\gamma$ on $\partial U$. Then $u-\gamma \leq v$ in $U$ implies $u \leq v$ in $U$ upon letting $\gamma \downarrow 0$. Hence we hereafter assume that $\gamma>0$ and

$$
u(x)-v(x) \leq-\gamma<0 \quad \text { on } \quad \partial U
$$

To derive a contradiction, we also assume that $x^{0} \in U$ and

$$
M_{0}:=u\left(x^{0}\right)-v\left(x^{0}\right)=\max \{u(x)-v(x): x \in U\}>0 .
$$

Then, by elementary calculus,

$$
D u\left(x^{0}\right)=D v\left(x^{0}\right) \quad \text { and } \quad D^{2} u\left(x^{0}\right) \leq D^{2} v\left(x^{0}\right) .
$$


Using (6.1), we also have

$$
\begin{aligned}
\Delta_{\infty} u\left(x^{0}\right) & =\left\langle D^{2} u\left(x^{0}\right) D u\left(x^{0}\right), D u\left(x^{0}\right)\right\rangle \geq 0, \\
\Delta_{\infty} v\left(x^{0}\right) & =\left\langle D^{2} v\left(x^{0}\right) D v\left(x^{0}\right), D v\left(x^{0}\right)\right\rangle \leq 0 .
\end{aligned}
$$

On the other hand, by (6.4),

$$
\begin{aligned}
\left\langle D^{2} u\left(x^{0}\right) D u\left(x^{0}\right), D u\left(x^{0}\right)\right\rangle & =\left\langle D^{2} u\left(x^{0}\right) D v\left(x^{0}\right), D v\left(x^{0}\right)\right\rangle \\
& \leq\left\langle D^{2} v\left(x^{0}\right) D v\left(x^{0}\right), D v\left(x^{0}\right)\right\rangle .
\end{aligned}
$$

There is no contradiction between (6.1) and the above relation. However, there would be a contradiction if one of the inequalities of (6.1) were strict at $x^{0}$.

To attempt to attain a strict inequality, we try a change of dependent variable. Let $0<\lambda$ be sufficiently small so that $2 \lambda u<1$. Define $w$ by $w \leq 1 / \lambda$ and

$$
u(x)=w(x)-\frac{\lambda}{2} w(x)^{2}=: G(w(x)) ;
$$

equivalently

$$
w=\frac{1}{\lambda}(1-\sqrt{1-2 \lambda u})=: H(u) .
$$

Clearly, $w \downarrow u$ uniformly as $\lambda \downarrow 0$. In view of (6.2) and (6.3), for small $\lambda, w \leq v$ on $\partial U$ and the maximum of $w-v$ over $\bar{U}$ is attained in $U$. Let us still denote such a maximum point by $x^{0}$.

Using (6.5), we find

$$
D u=G^{\prime}(w) D w, \quad D^{2} u=G^{\prime}(w) D^{2} w+G^{\prime \prime}(w)(D w \otimes D w)
$$

where $p \otimes q$ is the matrix defined by $(p \otimes q) z=\langle q, z\rangle p$. Thus

$$
\begin{aligned}
0 \leq & \Delta_{\infty} u=\left\langle D^{2} u D u, D u\right\rangle \\
& =\left\langle\left(G^{\prime \prime}(w)(D w \otimes D w)+G^{\prime}(w) D^{2} w\right) G^{\prime}(w) D w, G^{\prime}(w) D w\right\rangle \\
& =G^{\prime \prime}(w) G^{\prime}(w)^{2}|D w|^{4}+G^{\prime}(w)^{3}\left\langle D^{2} w D w, D w\right\rangle,
\end{aligned}
$$

which implies

$$
\Delta_{\infty} w=\left\langle D^{2} w D w, D w\right\rangle \geq-\frac{G^{\prime \prime}(w)}{G^{\prime}(w)}|D w|^{4}=\frac{\lambda}{1-\lambda w}|D w|^{4} .
$$

Hence, if $D w\left(x^{0}\right) \neq 0$, we have a contradiction, as then $\Delta_{\infty} w\left(x^{0}\right)>0$.

There are two main difficulties in turning the outline above into a proof. First, there is the fact that we know that viscosity solutions of $\Delta_{\infty} u=0$ need not be twice differentiable at every point (Examples 4.3 and 4.12). In fact, we do not even know if they have one derivative; all we know about subsolutions is that they are locally Lipschitz. Secondly, even at points where $w$ above might have two derivatives, we would not know that $D w \neq 0$. To overcome these difficulties is a demanding task. It involves:

- First $u, v$ will be approximated by better functions which still satisfy the hypotheses of the theorem (although $U$ will change a bit). This is done by a convexification process called "sup convolution" and provides new functions $u, v$ such that there is a constant $K$ for which $u(x)+(K / 2)|x|^{2}$ and $-v(x)+(K / 2)|x|^{2}$ are convex. One says that $u,-v$ are "semiconvex with constant $K$ "; one also says that $v$ is "semiconcave with constant $K$ ". This approximation process is presented in Section 6.1. 
- Regularity properties of convex functions and elements of the associated calculus of subdifferentials which will be presented. Of particular interest is that a semiconvex function is differentiable at its points of local maximum and, roughly speaking, the gradient is continuous at such a point. It is also the case that semiconvex and semiconcave functions are twice differentiable at almost all points so that the inequalities involving values of the infinity Laplace operator as derived in Section 4.4 can be used at these points. Finally, the points of twice differentiability may not include the maximum points, but this can be corrected by "perturbed optimization" - arbitrarily small linear perturbations can force this coincidence. The outline of the convex analysis theory we need is presented in Section 6.2. The perturbed optimization considerations appear in Section 6.3

- Roughly speaking, the argument breaks into cases depending on whether $D w$ vanishes at all points of maximum of $w-v$ (or something close to $w$ ) or not. If $D w$ is not zero at some maximum point of $w-v$ where one has twice differentiability, we are done. If it is zero-even more, if this is true for all small translations of $w$ - then Lemma2.13 comes into play, showing interior positive maxima force $w-v$ to be constant, which is again a contradiction. These considerations are also explained in Section 6.3.

For completeness of this orientation, we also recall the feature of the infinity Laplace equation illustrated by the functions

$$
u_{1}(x)=|x|, u_{2}(x)=x_{1} .
$$

Both functions are classical solutions of the infinity Laplace equation outside of the origin and $u_{1} \geq u_{2}$ everywhere. However, $u_{1}=u_{2}$ exactly on the half line $|x|=x_{1}$. Thus the maximum of $u_{2}-u_{1}$ over, say, $|x-(2,0, \ldots, 0)| \leq 1$ is 0 , and 0 is attained at every point of the line segment joining the antipodal points $(1,0, \ldots, 0)$ and $(3,0, \ldots, 0)$. That is, it is not true that the existence of interior maxima forces differences of classical solutions of the infinity Laplace equation to be constant.

We had better get going on the proofs. To reemphasize that $U$ is to be bounded throughout this section, we say it loudly:

\section{$U$ is bounded!}

Here is some notation that will be used concerning "perturbations" of the set $U$. We will have to both translate it and approximate it from inside. Translation will be denoted by $U^{h}$ and approximation from inside by $U_{\delta}$ where for $h \in \mathbb{R}^{n}$ and $0<\delta$

$$
U^{h}:=\{x-h: x \in U\}, \quad U_{\delta}:=\{x \in U: \operatorname{dist}(x, \partial U)>\delta\} .
$$

While we will return to the use of $w$ given by (6.5), for some time we will use it in other ways. That is,

$$
\text { until otherwise said, } w \text { is not necessarily given by (6.5)! }
$$

Let $w(x):=u(x+h)$. Clearly $u$ enjoys comparison with cones from above in $U$ iff $w$ enjoys comparison with cones from above on $U^{h}$. Operations on functions $w: U \rightarrow \mathbb{R}$, which we will apply to $u, v$ in Theorem 6.1 and which will require us to "move inside" $U$ a bit, are defined in the next section. Roughly speaking, before we are done, $U$ will be replaced by $U_{\delta} \cap U^{h}$ for suitable $\delta, h$. 


\subsection{Properties of Sup-Convolution.}

Definition 6.3. Let $w: U \rightarrow \mathbb{R}$ be bounded and $\epsilon>0$. Then, for $x \in \mathbb{R}^{n}$,

$$
w^{\epsilon}(x):=\sup _{y \in U}\left(w(y)-\frac{|x-y|^{2}}{2 \epsilon}\right)
$$

and

$$
w_{\epsilon}(x):=\inf _{y \in U}\left(w(y)+\frac{|x-y|^{2}}{2 \epsilon}\right) .
$$

Properties of $u^{\epsilon}$ (called the "sup-convolution" of $u$ ) are collected in the following proposition. Corresponding properties of $v_{\epsilon}$ (the "inf-convolution" of $v$ ) follow from obvious modifications in the proofs or from $v_{\epsilon}=-(-v)^{\epsilon}$. These properties will allow us to assume that $u$ is semiconvex (see the comments following the proof of the proposition) and $v$ is semiconcave when proving Theorem 6.1.

Proposition 6.4. Let $u: U \rightarrow \mathbb{R}$ satisfy $|u(x)| \leq A$ for $x \in U$. Then

$$
x \mapsto u^{\epsilon}(x)+\frac{1}{2 \epsilon}|x|^{2} \text { is convex on } \mathbb{R}^{n}
$$

and

$$
u^{\epsilon}(x) \geq u(x) \quad \text { for } \quad x \in U .
$$

If also $u \in \mathrm{CCA}(U)$ and $\delta>2 \sqrt{A \epsilon}$, then

$$
u^{\epsilon} \in \operatorname{CCA}\left(U_{\delta}\right) .
$$

Moreover,

$$
L_{u^{\epsilon}}(\bar{U}) \leq \frac{2 \sup _{y \in U}|y|}{\epsilon} .
$$

Finally, if $u \in C(\bar{U})$, then for $\delta>0$

$$
\lim _{\epsilon \downarrow 0} u^{\epsilon}(x)=u(x) \text { uniformly for } x \in U_{\delta} .
$$

Proof. We sketch the proofs. The assertion (6.12) holds because

$$
\begin{aligned}
u^{\epsilon}(x)+\frac{1}{2 \epsilon}|x|^{2} & =\sup _{y \in U}\left(u(y)-\frac{|x-y|^{2}}{2 \epsilon}+\frac{1}{2 \epsilon}|x|^{2}\right) \\
& =\sup _{y \in U}\left(u(y)-\frac{1}{2 \epsilon}|y|^{2}+\frac{1}{\epsilon}\langle x, y\rangle\right) .
\end{aligned}
$$

This exhibits the function in question as the supremum of linear (and hence convex) functions. Moreover, each of these functions has $\sup \{|y| / \epsilon: y \in U\}$ as a Lipschitz constant on $\mathbb{R}^{n}$, so $u^{\epsilon}(x)+(1 /(2 \epsilon))|x|^{2}$ does as well. This proves (6.12) and (6.15) (the latter upon inferring a Lipschitz constant for $u^{\epsilon}$ on $U$ from the previous observation). To prove $u^{\epsilon}(x) \geq u(x)$ if $x \in U$, note that since $x \in U, y=x$ is one of the choices of $y$ in the supremum defining $u^{\epsilon}(x)$. In view of this, the supremum is not altered if one restricts attention to those $y$ 's yielding at least the value $u(x)$, or

$$
u(y)-\frac{|x-y|^{2}}{2 \epsilon} \geq u(x) .
$$


Hence $|x-y|^{2} /(2 \epsilon) \leq u(y)-u(x) \leq 2 A$. Therefore, if $x \in U$, then

$$
u^{\epsilon}(x)=\sup _{\{y \in U,|x-y| \leq 2 \sqrt{A \epsilon}\}}\left(u(y)-\frac{|x-y|^{2}}{2 \epsilon}\right) .
$$

In consequence, if $\delta>2 \sqrt{A \epsilon}$ and $x \in U_{\delta}$, then

$$
u^{\epsilon}(x)=\sup _{\{|z| \leq 2 \sqrt{A \epsilon}\}}\left(u(x+z)-\frac{|z|^{2}}{2 \epsilon}\right)
$$

as follows from writing $z=y-x$ in (6.17). The assertion (6.14) is proved by noting that $x \mapsto u(x+z)-|z|^{2} /(2 \epsilon)$ is in $\mathrm{CCA}\left(U_{\delta}\right)$ if $u \in \mathrm{CCA}(U)$ and then recalling Lemma 2.12, It remains to explain (6.16). Since $u$ is continuous, when $\delta>2 \sqrt{A \epsilon}$ and $x \in U_{\delta}$, there is a point $z_{x},\left|z_{x}\right| \leq 2 \sqrt{A \epsilon}$ such that

$$
u^{\epsilon}(x)=u\left(x+z_{x}\right)-\frac{\left|z_{x}\right|^{2}}{2 \epsilon} \geq u(x) .
$$

Thus

$$
\begin{aligned}
\frac{\left|z_{x}\right|^{2}}{2 \epsilon} & \leq u\left(x+z_{x}\right)-u(x) \\
& \leq \sup \{|u(x+h)-u(x)|: x, x+h \in U,|h| \leq 2 \sqrt{A \epsilon}\}=: \rho(\epsilon)
\end{aligned}
$$

where $\lim _{\epsilon \downarrow 0} \rho(\epsilon)=0$ by the uniform continuity of $u$ on the compact set $\bar{U}$. Finally, there follows

$$
\begin{aligned}
\left|u^{\epsilon}(x)-u(x)\right| & =\left|u\left(x+z_{x}\right)-\frac{\left|z_{x}\right|^{2}}{2 \epsilon}-u(x)\right| \\
& \leq\left|u\left(x+z_{x}\right)-u(x)\right|+\frac{\left|z_{x}\right|^{2}}{2 \epsilon} \leq 2 \rho(\epsilon),
\end{aligned}
$$

establishing (6.16).

When $w: \mathbb{R}^{n} \rightarrow \mathbb{R}, K \in \mathbb{R}$ and

$$
x \mapsto w(x)+\frac{K}{2}|x|^{2}
$$

is convex, $w$ is said to be "semiconvex with constant $K$ ". Above, $u^{\epsilon}$ is semiconvex with constant $1 / \epsilon$. Similarly, $v_{\epsilon}$ is semiconcave with constant $1 / \epsilon$.

We return to the proof of Theorem 6.1. The proof consists of deriving a contradiction to the assumptions (6.1), (6.2) and (6.3). In view of Proposition6.4 (6.14), (6.16) and (6.2), (6.3), we can replace $u$ by $u^{\epsilon}, v$ by $v_{\epsilon}$ and $U$ by $U_{\delta}$ with suitable small $\delta>0, \epsilon>0$ and have all assumptions satisfied (although the constants $\gamma$, $M_{0}$ will be changed somewhat).

That is, we now assume $u \in \operatorname{CCA}(U) \cap C(\bar{U})$ is the restriction to $U$ of a semiconvex function on $\mathbb{R}^{n}, v \in \mathrm{CCB}(U) \cap C(\bar{U})$ is the restriction to $U$ of a function semiconcave on $\mathbb{R}^{n}$, and (6.1), (6.2), (6.3) hold. We seek a contradiction.

6.2. Elements of Convex Analysis. We will not use further notation to distinguish between $u$, as above, as a function on $\bar{U}$ and the semiconvex function on $\mathbb{R}^{n}$ of which it is a restriction (similarly for $v$ ). Here are some elementary facts about a continuous convex function $w: \mathbb{R}^{n} \rightarrow \mathbb{R}$. The epigraph of $w$

$$
\operatorname{epigraph}(w)=\left\{(x, r): x \in \mathbb{R}^{n}, r \geq w(x)\right\}
$$


is a closed convex subset of $\mathbb{R}^{n+1}$, and hence at each boundary point $(y, w(y))$ there is a supporting hyperplane. That is, there is a $p \in \mathbb{R}^{n}$ such that

$$
w(x) \geq w(y)+\langle p, x-y\rangle \text { for } \quad x \in \mathbb{R}^{n} .
$$

There may be many such $p$ 's (consider $n=1, w(x)=|x|, y=0$ ). The collection of them all is called $\partial w(y)$, the subdifferential of $w$ at $y$. That is,

$$
\partial w(y)=\left\{p \in \mathbb{R}^{n}:(6.19) \text { holds }\right\} .
$$

If $y^{j} \rightarrow y, p^{j} \rightarrow p, p^{j} \in \partial w\left(y^{j}\right)$, then passing to the limit in

$$
w(x) \geq w\left(y^{j}\right)+\left\langle p^{j}, x-y^{j}\right\rangle \quad \text { for } \quad x \in \mathbb{R}^{n},
$$

we find $p \in \partial w(y)$. This fact is referred to by saying that (the graph of) $\partial w$ is closed. In the event that $\partial w(y)$ contains exactly one element $p$, we say that $\partial w$ is single-valued at $y$ and write $\partial w(y)=p$. Since $\partial w$ is closed, if it is single-valued at $y$, then

$$
\lim _{r \downarrow 0} \sup _{x \in B_{r}(y)}\{|q-\partial w(y)|: q \in \partial w(x)\}=0 .
$$

This is called "partial continuity of the gradient". In more detail, this holds because if $p^{j} \in \partial w\left(x^{j}\right)$ and $x^{j} \rightarrow x$, then the $p^{j}$ are bounded (because convexity of $w$ implies it is locally Lipschitz continuous; alternatively, we have already noted the local Lipschitz continuity of the functions we are dealing with). The fact that $\partial w$ is closed then shows that every subsequence of the $p^{j}$ has a further subsequence converging to $\partial w(y)$, whence (6.20). Another fact is that if $\partial w$ is single-valued at $y$, then $w$ is differentiable at $y$ and $D w(y)=\partial w(y)$. Indeed, we have

$$
w(x) \geq w(y)+\langle\partial w(y), x-y\rangle
$$

and, for $q \in \partial w(x)$,

$$
\begin{aligned}
w(y) & \geq w(x)+\langle q, y-x\rangle \\
& =w(x)+\langle\partial w(y), y-x\rangle+\langle q-\partial w(y), y-x\rangle \\
& =w(x)+\langle\partial w(y), y-x\rangle+\mathrm{o}(|x-y|)
\end{aligned}
$$

as $x \rightarrow y$. The last estimate above used (6.20). Altogether, the two estimates imply

$$
w(x)=w(y)+\langle\partial w(y), x-y\rangle+\mathrm{o}(|x-y|)
$$

as $x \rightarrow y$, or $D w(y)=\partial w(y)$. Let us further note that (6.19) states that $y$ is a minimum of $x \mapsto w(x)-\langle p, x\rangle$. Thus if $w$ is differentiable at $y$, then $D w(y)=p$ and if $w$ is twice differentiable at $y$, then $D^{2} w(y) \geq 0$ (the $n \times n$ zero matrix).

What are the analogues of these facts for a semiconvex function $u$ with semiconvexity constant $K$ ? Let $w(x)=u(x)+K|x|^{2} / 2, u(x)=w(x)-K|x|^{2} / 2$. Then (6.19) written in terms of $u$ says

$$
u(x)+K \frac{|x|^{2}}{2} \geq u(y)+K \frac{|y|^{2}}{2}+\langle p, x-y\rangle \quad \text { for } \quad x \in \mathbb{R}^{n} .
$$

A little algebra transforms this into

or

$$
u(x) \geq u(y)-K \frac{|x-y|^{2}}{2}+\langle p-K y, x-y\rangle \quad \text { for } \quad x \in \mathbb{R}^{n},
$$

$$
u(x) \geq u(y)-K \frac{|x-y|^{2}}{2}+\langle q, x-y\rangle \quad \text { for } \quad x \in \mathbb{R}^{n},
$$


where $q=p-K y$. We take (6.21) as the definition of $q \in \partial u(y)$ :

$$
\partial u(y):=\left\{q \in \mathbb{R}^{n}: \text { (6.21) holds for } x \in \mathbb{R}^{n}\right\} .
$$

While the object defined involves $K$ and the notation doesn't reflect this, it is easy to see that, given that $u$ is semiconvex with some constant $K$ and hence with any larger constant, $\partial u(y)$ is independent of whatever number is chosen to be "the" semiconvexity constant. Simple modifications of the arguments for the convex case show the partial continuity of the gradient holds in the semiconvex case as does differentiability of $u$ where $\partial u$ is single-valued.

Let $u$ be semiconvex with constant $K$ on $\mathbb{R}^{n}$ and have a local maximum at $\hat{x}$, so that for some $r>0$,

$$
u(x) \leq u(\hat{x}) \text { for }|x-\hat{x}| \leq r .
$$

If $q \in \partial u(\hat{x})$ and $|x-\hat{x}| \leq r$, we also have

$$
\begin{aligned}
u(x) & \geq u(\hat{x})-\frac{K}{2}|x-\hat{x}|^{2}+\langle q, x-\hat{x}\rangle \\
& \geq u(x)-\frac{K}{2}|x-\hat{x}|^{2}+\langle q, x-\hat{x}\rangle .
\end{aligned}
$$

Thus $\langle q, x-\hat{x}\rangle \leq(K / 2)|x-\hat{x}|^{2}$, and this implies $q=0$. Thus $\partial u$ is single-valued at a local maximum $\hat{x}$ and $\partial u(\hat{x})=0$. If $u=u_{1}+u_{2}$ is a sum of two semiconvex functions, $\hat{x}$ is a local maximum of $u$ and $q^{j} \in \partial u_{j}(\hat{x}), j=1,2$, then it is easy to see that $q^{1}+q^{2} \in \partial u(\hat{x})$, so $q^{1}=-q^{2}$. Since this is true for any such pair, it implies there is only one pair $q^{1}, q^{2}$. Thus both $\partial u_{1}$ and $\partial u_{2}$ are single-valued at $\hat{x}$ and $\partial u_{1}(\hat{x})=-\partial u_{2}(\hat{x})$. Let us also note that if $u$ is semiconvex with constant $K$, then at points of twice differentiability $\hat{x}$,

$$
\left.D^{2}\left(u(x)+\frac{K}{2}|x|^{2}\right)\right|_{x=\hat{x}} \geq 0
$$

or

$$
D^{2} u(\hat{x}) \geq-K I \text {. }
$$

This is the reason " $K / 2$ " appears in the definition of semiconvexity.

6.3. The End Game. Recall now that we are assuming that $u,-v$ are semiconvex, Lipschitz on $\bar{U}$, and all of (6.1), (6.2), (6.3) hold. Choose any $\delta>0$ sufficiently small so that these assumptions hold with $U$ replaced by $U_{\delta}$ (with, perhaps, a slightly changed $\gamma$ ). If $|h|<\delta$, then $\left(U_{\delta}\right)^{h} \subset U$ and the functions $x \rightarrow u(x+h)$ will still satisfy their part of (6.1) on the new $U\left(=\right.$ old $\left.U_{\delta}\right)$, and are semiconvex with the same constant as $u$. Moreover, clearly,

$$
M(h):=\max _{x \in U}(u(x+h)-v(x)) \rightarrow M(0)=M_{0}>0 \quad \text { as } \quad h \rightarrow 0 .
$$

For any function $w: U \rightarrow \mathbb{R}$ we set

$$
\operatorname{Argmax}(w):=\left\{x \in U: w(x)=\max _{U} w\right\} .
$$

If $w \in C(U), w<0$ near $\partial U$, while $w(x)>0$ for some $x \in U, \operatorname{Argmax}(w)$ is nonempty. We also abuse notation a bit by putting

$$
\operatorname{Argmax}(h):=\{x \in U: M(h)=u(x+h)-v(x)\}=\operatorname{Argmax}(u(\cdot+h)-v(\cdot)) .
$$

Since $-v$ is semiconvex, as is $u, u(\cdot+h)$ and $v$ are both differentiable at every point of $\operatorname{Argmax}(h)$ and their derivatives are equal at those points (Section 6.2). 
We now assume that $U$ is connected. If it were not, we simply replace it by any of its components containing a maximum point of $u-v$. The proof of Theorem 6.1 now splits into two cases:

Case (i). There exists a $0<\kappa$ such that for $|h| \leq \kappa$ there exists $x_{h} \in \operatorname{Argmax}(h)$ such that $D u\left(x_{h}+h\right)=D v\left(x_{h}\right)=0$.

Case (ii). There exists $h$ sufficiently small such that (6.2) holds for $u(\cdot+h)$, $M(h)>0$, and $D u(x+h) \neq 0$ holds at all points $x \in \operatorname{Argmax}(h)$.

Proof of Theorem 6.1 in Case (i). Note that $M(h)$ is Lipschitz continuous in $h$. Let $x_{h}$ have the meaning assigned in Case (i) above. For $\left|h^{*}\right|<\delta$ we then have

$$
\begin{aligned}
M(h)=u\left(x_{h}+h\right)-v\left(x_{h}\right) & \geq u\left(x_{h^{*}}+h\right)-v\left(x_{h^{*}}\right) \\
& =u\left(x_{h^{*}}+h^{*}+h-h^{*}\right)-v\left(x_{h^{*}}\right) \\
& \geq u\left(x_{h^{*}}+h^{*}\right)-v\left(x_{h^{*}}\right)+o\left(\left|h^{*}-h\right|\right) \\
& =M\left(h^{*}\right)+o\left(\left|h^{*}-h\right|\right),
\end{aligned}
$$

where the first inequality is due to the definition of $x_{h}$, the second is due to $D u\left(x_{h^{*}}+h^{*}\right)=0$ and the last equality is due to the definition of $x_{h^{*}}$.

It follows that if $M$ is differentiable at $h^{*}$, then $D M\left(h^{*}\right)=0$. We rely on the reader to supply the fact that a Lipschitz continuous function whose derivative is 0 wherever it exists is constant on connected components of its domain. Thus $M$ is constant on $|h| \leq \kappa$. Now if $x_{0} \in \operatorname{Argmax}(0)$, we have

$$
u\left(x_{0}\right)-v\left(x_{0}\right)=M(0)=M(h)=u\left(x_{h}+h\right)-v\left(x_{h}\right) \geq u\left(x_{0}+h\right)-v\left(x_{0}\right),
$$

which implies that $x_{0}$ is a local maximum point of $u$. By Lemma2.13 $u$ is constant in a neighborhood of $x_{0}$. Since then, for $y$ sufficiently small,

$$
u\left(x_{0}\right)-v\left(x_{0}\right) \geq u\left(x_{0}+y\right)-v\left(x_{0}+y\right)=u\left(x_{0}\right)-v\left(x_{0}+y\right),
$$

$x_{0}$ is a local minimum of $v$ and then $v$ is constant in a neighborhood of $x_{0}$, as above. But then the set $\left\{x: u(x)-v(x)=u\left(x_{0}\right)-v\left(x_{0}\right)\right\}$ is open and closed, hence all of $U$. This contradicts (6.2).

Proof of Theorem 6.1 in Case (ii). Let $|h|<\delta$ be such that $D u(x+h) \neq 0$ for any $x \in \operatorname{Argmax}(h)$. Since $\operatorname{Argmax}(h)$ is closed, we have partial continuity of the gradient, and $D u(\cdot+h)$ does not vanish at any point of $\operatorname{Argmax}(h)$, there is a neighborhood $V$ of $\operatorname{Argmax}(h)$ such that $\operatorname{Argmax}(h) \subset V \subset \subset U$ and $\mu>0$ such that if $x \in V$ and $u(\cdot+h), v$ are both differentiable at $x$, then

$$
|D u(x+h)|,|D v(x)| \geq \mu>0 .
$$

Hereafter $h$ is fixed, so we replace $u(\cdot+h)$ by $u(\cdot)$ and drop the $h$ in the notation. Now, with our new abuse of notation,

$$
\operatorname{Argmax}(h)=\operatorname{Argmax}(u-v):=\left\{x \in U: u(x)-v(x)=\max _{U}(u-v)\right\} .
$$

Now we invoke the change of variables (6.5), (6.6). We claim that $w$ is semiconvex on $V$ because $u$ is semiconvex. This is seen via the formal computation

$$
D w=H^{\prime}(u) D u, \quad D^{2} w=H^{\prime}(u) D^{2} u+H^{\prime \prime}(u)(D u \otimes D u) \geq H^{\prime}(u) D^{2} u
$$

since $H$ is convex. Thus if $D^{2} u \geq-K I$, we have $D^{2} w \geq-H^{\prime}(u) K I$ and $H^{\prime} \geq 0$ is bounded on the range of $u$. A rigorous version of this is straightforward and left to 
the reader. Using (6.26), at any point of twice differentiability of $w$ (equivalently, $u$ ) in $V$, we have, via (6.26) and (6.8),

$$
\Delta_{\infty} w \geq \frac{\lambda}{1-\lambda w}|D w|^{4} \geq \frac{\lambda}{1-\lambda w} H^{\prime}(u)^{4} \mu^{4} \geq \beta
$$

where $\beta>0$ is a constant.

The discussion in this section has been self-contained up to this point, with the exception of the statement that a Lipschitz continuous function whose derivative is zero wherever it exists is locally constant. This was used in the proof of Case (i). Now we have to call on deeper results. First, according to a theorem of Aleksandrov, convex (and hence semiconvex) functions are twice differentiable in the pointwise sense almost everywhere. Next, as applied here, it is known that, in view of the semiconvexity, the Lebesgue measure of

$$
\bigcup_{|p| \leq \epsilon} \operatorname{Argmax}(x \mapsto w(x)-v(x)+\langle p, x\rangle)
$$

is positive for each $\epsilon>0$. Moreover, if $|p|$ and $\lambda>0$ are sufficiently small, we have

$$
\operatorname{Argmax}(x \mapsto w(x)-v(x)+\langle p, x\rangle) \subset V .
$$

Putting these facts together, we find that for each $\epsilon>0$, we have a $p \in \mathbb{R}^{n}$ and a point $\hat{x} \in V$ at which $w, v$ are both twice differentiable and, using the necessary conditions of calculus for a maximum point,

$$
|p| \leq \epsilon, \quad D w(\hat{x})+p=D v(\hat{x}) \quad \text { and } \quad D^{2} w(\hat{x}) \leq D^{2} v(\hat{x}) .
$$

See the Notes to this section for further information.

Further, if (6.31) holds and $K$ is a semiconvexity constant for $w,-v$, we have

$$
-K I \leq D^{2} w(\hat{x}) \leq D^{2} v(\hat{x}) \leq K I
$$

We then have

$$
\begin{aligned}
\left\langle D^{2} w(\hat{x})(D w(\hat{x})+p), D w(\hat{x})+p\right\rangle & =\left\langle D^{2} w(\hat{x}) D v(\hat{x}), D v(\hat{x})\right\rangle \\
& \leq\left\langle D^{2} v(\hat{x}) D v(\hat{x}), D v(\hat{x})\right\rangle \leq 0 .
\end{aligned}
$$

The last inequality is from (6.1) for $v$. On the other hand, using (6.28) and (6.32),

$$
\begin{aligned}
\left\langle D^{2} w(\hat{x})\right. & (D w(\hat{x})+p), D w(\hat{x})+p\rangle \\
= & \left\langle D^{2} w(\hat{x}) D w(\hat{x}), D w(\hat{x})\right\rangle+2\left\langle D^{2} w(\hat{x}) D w(\hat{x}), p\right\rangle+\left\langle D^{2} w(\hat{x}) p, p\right\rangle \\
& \geq \beta-2 K|D w(\hat{x})||p|-K|p|^{2} .
\end{aligned}
$$

As $|D w|$ is bounded on $V$, this is positive if $|p|$ is sufficiently small, whence a contradiction to (6.33).

Remark 6.5. It has been mentioned several times that the presentation of the uniqueness proof above did not invoke the notions of viscosity solution theory. For those who know something of this theory, we point out why this was possible: we derived the inequalities (6.1) at points of twice differentiability in Section 4.4 without reference to viscosity solutions. Moreover, we proved directly that the sup-convolution of a function in CCA was again in CCA in Section 6.1 
Remark 6.6. In order to prove the uniqueness Theorem 6.1 in the case of a norm other than the Euclidean norm, one uses the inequalities proved in Section 5 If $J$ is single-valued, the arguments above all are valid with

$$
\Delta_{|\cdot|} u=\left\langle D^{2} u J(D u), J(D u)\right\rangle
$$

in place of $\Delta_{\infty}$. However, the Euclidean norm is still to be used in defining the sup-convolutions, etc. The key point is the analogue of (6.7) in this case. Since

$$
\begin{aligned}
\left\langle(D w \otimes D w) J\left(G^{\prime}(D w) D w\right), J\left(G^{\prime}(D w) D w\right)\right\rangle & =\langle(D w \otimes D w) J(D w), J(D w)\rangle \\
& =\langle D w, J(D w)\rangle^{2}=|D w|^{2}
\end{aligned}
$$

by Remark 5.1 the analogue of 6.7 is

$$
\Delta_{|\cdot|} w \geq \frac{\lambda}{1-\lambda w}|D w|^{2}
$$

and the rest of the proof runs as before. A primary difficulty with the case in which $J$ is not single-valued is this: we cannot say at a point of maximum $x^{0}$ of $u-v$ at which both $u$ and $v$ are differentiable that, necessarily,

$$
\text { " }\left\langle D^{2} u J(D u), J(D u)\right\rangle \geq 0 \geq\left\langle D^{2} v J(D v), J(D v)\right\rangle ",
$$

since we only have each inequality upon choosing the "right value" of the duality mapping on each side and these values may differ even if $u$ is smooth and $u=v$. See Example 5.2 .

Remark 6.7. Here is a fact about viscosity subsolutions of a degenerate elliptic equation $F\left(D u, D^{2} u\right) \leq 0$ which is nonincreasing in $D^{2} u$ (see [32] for the terminology). If $u$ is semiconvex, then it is a viscosity subsolution iff $F\left(D u(x), D^{2} u(x)\right) \leq 0$ at each point $x$ of twice differentiability of $u$. As shown above, if $u \in \operatorname{CCA}(U)$, then $u^{\epsilon} \in \mathrm{CCA}\left(U_{\delta}\right)$. Thus $u^{\epsilon}$ satisfies $\Delta_{|\cdot|} u^{\epsilon} \leq 0$ at points of twice differentiability if $J$ is single-valued. But then it is a viscosity solution of $\Delta_{|\cdot|} u^{\epsilon} \leq 0$ and then so is its limit $u$.

6.4. Notes. After the foundational papers of Aronsson, the uniqueness of absolutely minimizing extensions remained as the most important open problem. The issue was settled in Jensen's influential work [4]; he proved the comparison principle for viscosity sub- and supersolutions. Jensen's proof of the comparison principle was rather indirect in the sense that the result was first established for two auxiliary equations approximating (0.7) in a suitable manner. These approximations were introduced to overcome the difficulties associated with vanishing gradients, and Sobolev inequalities came into play in passage to the original equation. Moreover, the arguments relied on the theory of variational problems in $L^{p}$. Jensen used the change of variables employed above; this is a standard device for introducing strictness. It was used, for example, by Crandall and Lions in [31] to prove uniqueness for viscosity solutions of eikonal equations.

A more direct uniqueness proof, independent of the $L^{p}$ theory, was found by Barles and Busca [12. Their main contribution was perhaps to introduce the dichotomy of Cases (i) and (ii) and then to handle Case (i). We have used their arguments to handle this case. The treatment of Case (ii) is standard from the point of view of viscosity solution theory, although we have made our presentation independent of that theory. 
Both the proof of Jensen and the one by Barles and Busca work for more general equations than just the infinity Laplace equation, and their ideas have been extended to various settings. See, for example, [64], [48], [19], [20], [50] and [62].

Finally, let us provide some references for the tools we employed in the proof. The almost everywhere twice differentiability of convex functions, known as Aleksandrov's theorem, can be found in [36] or [32]. The statement concerning the Lebesgue measure of the set (6.29) is known as "Jensen's lemma" owing to [46]. Jensen recognized that this sort of fact was valid for semiconvex functions. The proofs are, however, basically the ones used in establishing the "Aleksandrov, Bakelmann, Pucci" maximum principle. See, for example, [23] or [41]. A proof and further references are also available in 32 .

\section{Regularity}

We note that the term "regularity" is a catch-all for properties speaking to the modulus of continuity of a function, differentiability properties of it and estimates on the size and continuity of derivatives. So far we have presented a variety of results bearing on the regularity of an absolutely minimizing function. One is the Lipschitz estimate of Lemma 2.9; another is the refinement of the Harnack inequality presented in Section 2.6. The rough thrust of these estimates and others herein is that if $u \in \mathrm{CCA}(U)$, then it is locally Lipschitz continuous and one can estimate $\mathrm{T}_{u}(x)$ in terms of estimates of $u$ itself. Local Lipschitz continuity implies differentiability almost everywhere, and one can easily show that $T_{u}(x) \geq|D u(x)|^{*}$ when $u$ is differentiable at $x$. One might also regard Lemma 2.13 as speaking to regularity in some way. All of these results are true under "one-sided" assumptions and in any norm. In addition, we have Example 4.12 which shows that, when $|\cdot|$ is the Euclidean norm, merely assuming $u \in \mathrm{AM}(U)$ cannot possibly imply more than continuous differentiability of $u$ in $U$ and that $D u$ is locally Hölder continuous with exponent $1 / 3$. It is not known, at the moment, that this is false. It is also not known, at the moment, whether or not $u \in \operatorname{AM}(U)$ implies that $u$ is differentiable at every point of $U$ in the case of the Euclidean norm except when $n=2$. More generally, taking $u(x)=|x|$, we recall that $u$ is not differentiable at every point of $\mathbb{R}^{n} \backslash\{0\}$ unless $|\cdot|$ is differentiable; in particular $|\cdot|_{1},|\cdot|_{\infty}$ do not have this property.

As of this writing, the one and only regularity result known to us using the full assumption $u \in \operatorname{AM}(U)$ which is valid in any dimension is Theorem 7.1 below. The proof is given in the case of the Euclidean norm. Modifications extend the arguments to norms which, together with their dual norms, are strictly convex. The theorem is false for general norms, as the example $u(x)=|x|_{\infty}$ shows when $|\cdot|_{\infty}$

Theorem 7.1. Let $u \in \operatorname{AM}(U)$ and $x^{0} \in U$. Let $|\cdot|$ be the Euclidean norm. If $\lambda_{j}>0$ satisfies $\lambda_{j} \downarrow 0, v \in C\left(\mathbb{R}^{n}\right)$, and

$$
v(x)=\lim _{j \rightarrow \infty} \frac{u\left(\lambda_{j} x+x^{0}\right)-u\left(x^{0}\right)}{\lambda_{j}}
$$

holds uniformly on compact subsets of $\mathbb{R}^{n}$, then $v$ is a linear function.

Remark 7.2. Since the initial preparation of this manuscript, it has been shown by O. Savin [61] that if $n=2$ is added to the assumptions of Theorem [7.1, then 
$u \in C^{1}(U)$. Savin began from an appealing reformulation of Theorem 7.1

$$
\lim _{r \downarrow 0} \inf _{\left\{e \in \mathbb{R}^{n}:|e|=T_{u}(0)\right\}} \sup _{|x| \leq r} \frac{\left|u\left(x+x^{0}\right)-u\left(x^{0}\right)-\langle e, x\rangle\right|}{r}=0 .
$$

After the proof of the theorem, the reader will easily see the two formulations are equivalent.

Let us explain the significance of Theorem 7.1. If $u$ is differentiable at $x^{0}$ in the sense of (1.5) of Section 1.5, then a computation shows that

$$
\frac{u\left(\lambda x+x^{0}\right)-u\left(x^{0}\right)}{\lambda}=\left\langle D u\left(x^{0}\right), x\right\rangle+\mathrm{o}(1)
$$

as $\lambda \downarrow 0$. Hence the same limit $v$ is obtained in (7.1) no matter what sequence $\lambda_{j} \downarrow 0$ is used and $v(x)=\left\langle D u\left(x^{0}\right), x\right\rangle$ is linear. We will shortly see that it is always true that the functions $v_{\lambda}(x):=\left(u\left(\lambda x+x^{0}\right)-u\left(x^{0}\right)\right) / \lambda$ form a precompact family in $C\left(B_{R}(0)\right)$ for $R>0$ as soon as $\lambda$ is sufficiently small. This depends only on the local Lipschitz continuity of $u$. Thus, if $\lambda_{j}$ is any sequence such that $\lambda_{j} \downarrow 0$, then there is a subsequence $\lambda_{j_{k}}$ such that $v_{\lambda_{j_{k}}}$ will converge locally uniformly to a limit $v$ on $\mathbb{R}^{n}$. The theorem asserts that $v$ must be linear, which we have seen must be the case if $D u\left(x^{0}\right)$ exists. However, we do not know if the limit $v$ in (7.1) depends on the particular sequence via which it is defined. If it does not, then it is easy to see that $D u\left(x^{0}\right)$ exists and $D u\left(x^{0}\right)=D v(0)$. Indeed, if $v(x)=\langle p, x\rangle$ no matter which sequence is used, but $D u\left(x^{0}\right)$ does not exist, then there are an $\epsilon>0$ and a sequence $x^{j} \rightarrow 0$ such that

$$
\frac{\left|u\left(x^{j}+x^{0}\right)-u\left(x^{0}\right)-v\left(x^{j}\right)\right|}{\left|x^{j}\right|} \geq \epsilon \quad \text { for } \quad j=1,2 \ldots .
$$

Putting $\lambda_{j}=\left|x^{j}\right|$ and $\omega^{j}=x^{j} / \lambda_{j},(7.2)$ says

$$
\left|\frac{u\left(\lambda_{j} \omega^{j}+x^{0}\right)-u\left(x^{0}\right)}{\lambda_{j}}-v\left(\omega^{j}\right)\right| \geq \epsilon \quad \text { for } \quad j=1,2 \ldots .
$$

Thus $\lambda_{j}$ has no subsequence along which $\left(u\left(\lambda_{j} x+x^{0}\right)-u\left(x^{0}\right)\right) / \lambda_{j}$ converges uniformly to $v(x)$ on $|x|=1$.

Thus Theorem 7.1 provides positive, but not definitive, evidence that $D u\left(x^{0}\right)$ exists. It remains an open problem to determine if absolutely minimizing functions are everywhere differentiable. We turn to the proof.

Proof. First we dispose of the case

$$
S_{u}^{+}\left(x^{0}\right)=T_{u}\left(x^{0}\right)=0 .
$$

We are using Lemma 2.15 (iv) and, during the current discussion, indexing $S^{+}$by the function used to compute it. In this case,

$$
\left|u(x)-u\left(x^{0}\right)\right| \leq \mathrm{L}_{u}\left(B_{\lambda}\left(x^{0}\right)\right)\left|x-x^{0}\right| \quad \text { for } \quad\left|x-x^{0}\right| \leq \lambda,
$$

so $u(x)=u\left(x^{0}\right)+\mathrm{o}\left(\left|x-x^{0}\right|\right)$. This shows that $D u\left(x^{0}\right)$ exists and $D u\left(x^{0}\right)=0$. Hereafter we assume that

$$
L_{0}:=S_{u}^{+}\left(x^{0}\right)=\mathrm{T}_{u}\left(x_{0}\right)>0 .
$$


If $u \in \mathrm{CC}(U), x^{0} \in U, B_{r_{0}}\left(x^{0}\right) \subset \subset U$ and $\tilde{u}(x)=\left(u\left(r_{0} x+x^{0}\right)-u\left(x^{0}\right)\right) /\left(r_{0} L_{0}\right)$, then $\tilde{u} \in \mathrm{CC}\left(B_{1}(0)\right), \tilde{u}(0)=0$ and $S_{\tilde{u}}^{+}(0)=S_{u}^{+}\left(x^{0}\right) / L_{0}=1$. Hereafter, we simply assume that

$$
u \in \mathrm{CC}\left(B_{1}(0)\right), \quad u(0)=0, \quad S_{u}^{+}(0)=1 .
$$

Given (7.3), for $\lambda>0$ the function

$$
v_{\lambda}(x):=\frac{u(\lambda x)}{\lambda}
$$

satisfies $v_{\lambda} \in \mathrm{CC}\left(B_{1 / \lambda}(0)\right), v_{\lambda}(0)=0$ and for $r<1 / \lambda$,

$$
\mathrm{L}_{v_{\lambda}}\left(B_{r}(0)\right)=\mathrm{L}_{u}\left(B_{\lambda r}(0)\right), \quad \max _{|w|=r} v_{\lambda}(w)=\frac{\max _{|w|=\lambda r} u(w)}{\lambda}=r S_{u}^{+}(0, \lambda r) .
$$

Thus the family $v_{\lambda}$ is uniformly bounded and equicontinuous in each ball $B_{r}(0)$ as $\lambda \downarrow 0$. Therefore there exists a sequence $\lambda_{j} \downarrow 0$ and $v \in C\left(\mathbb{R}^{n}\right)$ such that $v_{\lambda_{j}} \rightarrow v$ uniformly on every bounded set. Clearly $v \in \mathrm{CC}\left(\mathbb{R}^{n}\right)$ (comparison with cones is obviously preserved under uniform convergence). Putting $\lambda=\lambda_{j}$ in the relations above and passing to the limit then yields the first two claims below:

$$
\mathrm{L}_{v}\left(B_{r}(0)\right) \leq \mathrm{T}_{u}(0)=1, \quad \max _{|w|=r} v(w)=r, \quad \min _{|w|=r} v(w)=-r .
$$

Here we used the simple facts that Lipschitz constants are upper-semicontinuous and maxima are continuous with respect to uniform convergence. The third relation above is asserted without proof, but its proof is evident. Note that the first relation, being valid for $r>0$, implies $\mathrm{L}_{v}\left(\mathbb{R}^{n}\right) \leq 1$.

The properties (7.5), valid for $r>0$, together with $v(0)=0$, guarantee that $v$ is linear. To see this, let $x_{r}^{+}, x_{r}^{-}$be any two points for which

$$
\left|x_{r}^{+}\right|,\left|x_{r}^{-}\right|=r \text { and } v\left(x_{r}^{+}\right)=r, v\left(x_{r}^{-}\right)=-r .
$$

Until this point we have not used the assumption that $|\cdot|$ is the Euclidean norm. Now we do: being points in the sphere of radius $r$ about 0 , unless $x_{r}^{+}=-x_{r}^{-}$we will have $\left|x_{r}^{+}-x_{r}^{-}\right|<2 r$, in which case (7.6) is inconsistent with $2 r=v\left(x_{r}^{+}\right)-v\left(x_{r}^{-}\right) \leq$ $\left|x_{r}^{+}-x_{r}^{-}\right|$. Here we used that $\mathrm{L}_{v}\left(\mathbb{R}^{n}\right) \leq 1$. We have now proved that the points $x_{r}^{+}, x_{r}^{-}$satisfying (7.6) are unique. This is because $x_{r}^{+}=-x_{r}^{-}$must hold for any such pair.

Next we claim that $v$ is linear on the line segment $\left[x_{r}^{-}, x_{r}^{+}\right]$; that is,

$$
g(t):=v\left(t x_{r}^{+}\right)=v\left(-t x_{r}^{-}\right)=t r \text { for }-1 \leq t \leq 1 \text {. }
$$

Indeed, $g(-1)=-r=r(|-1+1|-1), g(1)=r=r(|1+1|-1)$, and $r$ is a Lipschitz constant for $g$. That is, $g(t)$ agrees with the cone function $t \mapsto r(|t+1|-1)=r t$ on the boundary of $U=(-1,1)$ and has the same Lipschitz constant, so (via Section 1.4) $g(t) \equiv t r$. It follows that all the points $x_{r}^{+}, x_{r}^{-}$lie on the same line.

Let $x^{2}, \ldots, x^{n}$ be such that $x_{1}^{+}, x^{2}, \ldots, x^{n}$ is an orthonormal basis of $\mathbb{R}^{n}$ and define $f: \mathbb{R}^{n} \rightarrow \mathbb{R}$ by

$$
f\left(x_{1}, y\right):=f\left(x_{1}, y_{2}, \ldots, y_{n}\right)=v\left(x_{1} x_{1}^{+}+y_{2} x^{2}+\ldots+y_{n} x^{n}\right)
$$


where $y=\left(y_{2}, \ldots, y_{n}\right)$. Clearly, $\mathrm{L}_{f}\left(\mathbb{R}^{n}\right)=\mathrm{L}_{v}\left(\mathbb{R}^{n}\right)=1$ and $f\left(x_{1}, 0\right) \equiv x_{1}$. We claim then that $f\left(x_{1}, y\right) \equiv x_{1}$. To see this, first observe that

$$
\begin{aligned}
|y|^{2}+\left|x_{1}-s\right|^{2} & \geq\left|f\left(x_{1}, y\right)-f(s, 0)\right|^{2} \\
& =\left|f\left(x_{1}, y\right)-x_{1}+x_{1}-f(s, 0)\right|^{2} \\
& =\left|f\left(x_{1}, y\right)-x_{1}+x_{1}-s\right|^{2} \\
& =\left|f\left(x_{1}, y\right)-x_{1}\right|^{2}+2\left(x_{1}-s\right)\left(f\left(x_{1}, y\right)-x_{1}\right)+\left|x_{1}-s\right|^{2} .
\end{aligned}
$$

The first inequality above is due to $\mathrm{L}_{f}\left(\mathbb{R}^{n}\right) \leq 1$. We conclude that

$$
2\left(x_{1}-s\right)\left(f\left(x_{1}, y\right)-x_{1}\right) \leq|y|^{2}
$$

where $s$ is free. This can only be if $f\left(x_{1}, y\right)-x_{1} \equiv 0$. It follows that $v$ is linear.

Remark 7.3. From the proof above, $|D v|=\mathrm{T}_{u}(0)$ and the direction of $D v$ is the limiting direction of $w_{\lambda_{j}}$, where $w_{r}$ is any point of maximum of $u$ on $\left\{w:\left|w-x^{0}\right|=\right.$ $r\}$. Thus if $\mathrm{T}_{u}\left(x^{0}\right)>0, u$ is differentiable at 0 if and only if $\left(w_{r}-x^{0}\right) / r$ has a limit as $r \downarrow 0$ which is independent of the choice of $w_{r}$.

7.1. Notes. As explained above, the exact regularity of absolutely minimizing functions is largely an open problem. Theorem [7.1 is the only positive result we know of for a generic absolute minimizer. It originally appeared in [30]. Under certain additional convexity and monotonicity assumptions, some results compensating the missing continuity of the gradient have been established in [54. We want to point out that the lack of regularity in all of the examples we have presented in this work is not due to the gradient being zero in any sense.

\section{Appendix: $L^{p}$ VARiational PROBlems}

As we have already mentioned several times, a popular technique for studying absolute minimizers goes through an approximation process involving related variational problems in integral form. The purpose of this appendix is to sketch briefly this approach and explain how it relates to the material in the main text. The discussion will be far less self-contained than in the preceding sections. In particular, we need to appeal to several facts in functional analysis and the theory of Sobolev spaces. The reader should compare the machinery used in this line of attack to that of the previous sections.

For simplicity, it is assumed throughout this section that $U$ is a bounded open set. In addition, we suppose that the mapping $x \mapsto|x|^{2}$ is differentiable. By convex analysis, this guarantees that the dual norm $|x|^{*}$ is strictly convex; that is, if $x, y \in \mathbb{R}^{n}$ do not lie on a common ray through the origin, then

$$
|t x+(1-t) y|^{*}<t|x|^{*}+(1-t)|y|^{*} \quad \text { for all } 0<t<1 .
$$

8.1. Sobolev spaces. For a smooth, real-valued function $u \in C^{\infty}(U)$ and $1<p<$ $\infty$, define

$$
\|u\|_{1, p}:=\left(\int_{U}|u(x)|^{p} d x\right)^{1 / p}+\left(\int_{U}\left(|D u(x)|^{*}\right)^{p} d x\right)^{1 / p} .
$$

Here $D u$ is the gradient of $u, d x$ stands for the usual $n$-dimensional Lebesgue measure and $|\cdot|^{*}$ is the norm dual to $|\cdot|$ defined in Section 1.1. Then the Sobolev space $W^{1, p}(U)$ is defined as the completion of $C^{\infty}(U)$ in the norm $\|\cdot\|_{1, p}$. By the theorem of Meyers and Serrin, $u \in W^{1, p}(U)$ if and only if $u$ and all its weak first 
order partial derivatives (in the sense of distributions) are elements of the Lebesgue space $L^{p}(U)$. $W^{1, p}(U)$ is a reflexive Banach space for any $p$ and a Hilbert space for $p=2$.

An important subspace of $W^{1, p}(U)$ is the closure of $C_{0}^{\infty}(U)$, the set of smooth compactly supported functions, in the norm $\|\cdot\|_{1, p}$. It is denoted by $W_{0}^{1, p}(U)$, and heuristically it consists of those Sobolev functions that vanish on $\partial U$. Furthermore, for a given $w \in W^{1, p}(U)$, we denote

$$
W_{w}^{1, p}(U)=\left\{u \in W^{1, p}(U): u-w \in W_{0}^{1, p}(U)\right\} .
$$

Since $W_{w}^{1, p}(U)$ is closed and convex, it is also weakly closed by Mazur's lemma. We will need the following facts about Sobolev functions:

- If $1<q \leq p<\infty$, then $W^{1, p}(U) \subset W^{1, q}(U)$. This follows from Hölder's inequality and the boundedness of $U$.

- If $u$ is Lipschitz continuous in $U$, then $u \in W^{1, p}(U)$ for every $p$.

- There is a constant $C$ depending on $n, p$ and $U$ such that

$$
\|u\|_{1, p} \leq C\left(\int_{U}\left(|D u(x)|^{*}\right)^{p} d x\right)^{1 / p}
$$

for all $u \in W_{0}^{1, p}(U)$. This is a special case of the Sobolev embedding theorem.

- If $u \in W^{1, p}(U)$ and $p>n$, then $u$ is locally Hölder continuous in $U$. Moreover, if $u \in W_{0}^{1, p}(U)$, then $u$ is Hölder continuous in $U$ and

$$
|u(x)-u(y)| \leq C\left(\int_{U}\left(|D u(x)|^{*}\right)^{p} d x\right)^{1 / p}|x-y|^{1-n / p}
$$

for all $x, y \in U$ and for some constant $C$ depending on $n, p$ and $U$.

8.2. $p$-harmonic functions. We consider the problem of minimizing the functional

$$
I_{p}(u)=\int_{U}\left(|D u|^{*}\right)^{p} d x
$$

in the affine subspace $W_{w}^{1, p}(U)$. Since $\hat{I}_{p}:=\inf \left\{I_{p}(u): u \in W_{w}^{1, p}(U)\right\}$ is finite, there is a minimizing sequence $v_{j}$ such that $I_{p}\left(v_{j}\right) \rightarrow \hat{I}_{p}$ as $j \rightarrow \infty$. In particular, by (8.2), the sequence $v_{j}$ is bounded in $W^{1, p}(U)$. Thus there exists $u_{p} \in W^{1, p}(U)$ such that, up to a subsequence, $v_{j} \rightarrow u_{p}$ in the weak topology. As $W_{w}^{1, p}(U)$ is weakly closed, $u_{p} \in W_{w}^{1, p}(U)$. On the other hand, by the weak lower semicontinuity of norms,

$$
I_{p}\left(u_{p}\right)=\int_{U}\left(\left|D u_{p}\right|^{*}\right)^{p} d x \leq \liminf _{j \rightarrow \infty} \int_{U}\left(\left|D v_{j}\right|^{*}\right)^{p} d x=\liminf _{j \rightarrow \infty} I_{p}\left(v_{j}\right)=\hat{I}_{p} .
$$

Thus $u_{p}$ is a minimizer of (8.4) over $W_{w}^{1, p}(U)$. Such a function is called a generalized p-harmonic function with boundary values $w$; observe that the "boundary values" are attained, at least a priori, only in the weak sense $u_{p}-w \in W_{0}^{1, p}(U)$. By the regularity theory of elliptic partial differential equations, every $p$-harmonic function is continuous. (Alternatively, the reader may as well suppose that $p>n$, in which case every Sobolev function has a continuous representative.) The name "generalized $p$-harmonic function" stems from the fact that if $p=2$ and the norm is Euclidean, we recover the ordinary harmonic functions. 
The property of being $p$-harmonic is local in the sense that $u$ is $p$-harmonic in $U$ iff $u$ is $p$-harmonic in $V$ (with boundary values $u$ ) for every open $V \subset U$. This follows easily from the set additivity of the functional $I_{p}$ and the fact that if $v \in W_{u}^{1, p}(V)$ for some open $V \subset U$, then the function

$$
\tilde{u}= \begin{cases}u, & \text { in } U \backslash V, \\ v, & \text { in } V,\end{cases}
$$

is in $W_{u}^{1, p}(U)$. The reader should recall that this property is not true for the minimal Lipschitz extensions and that imposing it led to the concept of absolute minimizers.

The uniqueness of a $p$-harmonic function with given boundary values $w$ follows from the strict convexity of $I_{p}$. Indeed, let $u_{1}$ and $u_{2}$ be $p$-harmonic in $U$ so that $u_{1}-u_{2} \in W_{0}^{1, p}(U)$; that is, $u_{1}=u_{2}$ on $\partial U$ in the sense explained above. If the set where $u_{1} \neq u_{2}$ has positive measure, then, by the strict convexity of $|\cdot|^{*}$,

$$
\hat{I}_{p} \leq I_{p}\left(\frac{1}{2}\left(u_{1}+u_{2}\right)\right)<\frac{1}{2}\left(I_{p}\left(u_{1}\right)+I_{p}\left(u_{2}\right)\right)=\hat{I}_{p}
$$

which is impossible. Thus $u_{1}=u_{2}$ in $U$. As a rather immediate consequence of the uniqueness, we note further that $u_{1} \leq u_{2}$ on $\partial U$ (which really means that $\left.\max \left\{u_{1}-u_{2}, 0\right\} \in W_{0}^{1, p}(U)\right)$ implies $u_{1} \leq u_{2}$ on $U$. This follows by noting that if the open set

$$
V=\left\{x: u_{1}(x)>u_{2}(x)\right\}
$$

were not empty, then we would have two distinct $p$-harmonic functions with the same boundary values in $V$, violating the uniqueness. Observing further that $u+c$ is $p$-harmonic for any $c \in \mathbb{R}$ if $u$ is, we conclude that the estimate

$$
\sup _{x \in U}\left(u_{1}-u_{2}\right)=\sup _{x \in \partial U}\left(u_{1}-u_{2}\right)
$$

is valid for $p$-harmonic functions $u_{1}, u_{2} \in W^{1, p}(U) \cap C(\bar{U})$.

8.3. Connection to Lipschitz extensions. Let $f: \partial U \rightarrow \mathbb{R}$ be so that $\mathrm{L}_{f}(\partial U)$ is finite, and let $w: \bar{U} \rightarrow \mathbb{R}$ be any Lipschitz continuous function satisfying $w=f$ on $\partial U$. Since $w \in W^{1, p}(U)$ for any $1<p<\infty$, there exist $p$-harmonic functions $u_{p} \in W_{w}^{1, p}(U)$. By Hölder's inequality and the definition of $p$-harmonic functions,

$$
\begin{aligned}
\left(\int_{U}\left(\left|D u_{p}(x)\right|^{*}\right)^{q} d x\right)^{1 / q} & \leq|U|^{1 / q-1 / p}\left(\int_{U}\left(\left|D u_{p}(x)\right|^{*}\right)^{p} d x\right)^{1 / p} \\
& \leq|U|^{1 / q-1 / p}\left(\int_{U}\left(|D w(x)|^{*}\right)^{p} d x\right)^{1 / p} \\
& \leq|U|^{1 / q} \underset{x \in U}{\operatorname{ess} \sup }|D w(x)|^{*}
\end{aligned}
$$

for a fixed $1<q<\infty$ and $p \geq q$. Here $|U|$ denotes the Lebesgue measure of $U$. The above estimate implies that $\left\{u_{p}\right\}_{p \geq q}$ is bounded in $W^{1, q}(U)$, and thus it contains a weakly convergent subsequence, still denoted by $\left(u_{p}\right)$, converging to a function $u \in W_{w}^{1, q}(U)$. We deduce using the weak lower semicontinuity of norms that

$$
\left(\int_{U}\left(|D u(x)|^{*}\right)^{q} d x\right)^{1 / q} \leq|U|^{1 / q} \operatorname{ess~sup}_{x \in U}|D w(x)|^{*} .
$$

The same reasoning can be repeated for $q+1, q+2, \ldots$, so that in each step we select the weakly convergent sequence as a subsequence of the previous one. Taking 
the diagonal sequence ensures that we have found a subsequence $\left(u_{p_{j}}\right)$ such that $u_{p_{j}} \rightarrow u$ weakly in $W^{1, q}(U)$ and (8.6) holds for any $q<\infty$. Hence, letting $q \rightarrow \infty$,

$$
\underset{x \in U}{\operatorname{ess} \sup }|D u(x)|^{*} \leq \underset{x \in U}{\operatorname{ess} \sup }|D w(x)|^{*},
$$

and this is true for any Lipschitz function $w$ with $w=f$ on $\partial U$. Observe further that by using (8.3), (8.6) and Arzela-Ascoli, we may assume that $u_{p_{j}} \rightarrow u$ uniformly in $U$.

To see that $u$ has also the corresponding absolutely minimizing property, let us take $V \subset \subset U$ and a Lipschitz function $v$ on $V$ such that $u=v$ on $\partial V$. Then we can repeat the above construction and find a sequence of $p$-harmonic functions $\left(v_{p_{j_{k}}}\right)$ on $V$, agreeing with $v$ on $\partial V$, such that $v_{p_{j_{k}}} \rightarrow \tilde{v}$ and

$$
\underset{x \in V}{\operatorname{ess} \sup }|D \tilde{v}(x)|^{*} \leq \underset{x \in V}{\operatorname{essup}}|D v(x)|^{*} .
$$

By (8.5), since both $v_{p_{j_{k}}}$ and $u_{p_{j_{k}}}$ are $p_{j_{k}}$-harmonic in $V$, we have

$$
\begin{aligned}
\sup _{x \in V}|u(x)-\tilde{v}(x)| & \leq \sup _{x \in V}\left|u(x)-u_{p_{j_{k}}}(x)\right|+\sup _{x \in \partial V}\left|u_{p_{j_{k}}}(x)-v_{p_{j_{k}}}(x)\right| \\
& +\sup _{x \in V}\left|\tilde{v}(x)-v_{p_{j_{k}}}(x)\right| .
\end{aligned}
$$

As $u_{p_{j}} \rightarrow u$ and $v_{p_{j_{k}}} \rightarrow \tilde{v}$ uniformly in $V$ and $u=\tilde{v}$ on $\partial V$, this implies that $u=\tilde{v}$ in $V$. In particular,

$$
\underset{x \in V}{\operatorname{ess} \sup _{0}}|D u(x)|^{*} \leq \underset{x \in V}{\operatorname{ess} \sup }|D v(x)|^{*}
$$

for any Lipschitz continuous $v \in C(\bar{V})$ that agrees with $u$ on $\partial V$. Hence, using the results in Section 4, we have established that $u$ is an absolute minimizer in $U$. Moreover, if the duality map $J$ is single-valued, it follows from the uniqueness of absolute minimizers that the entire sequence $\left\{u_{p}\right\}$ of generalized $p$-harmonic functions converges uniformly to the absolute minimizer.

8.4. Euler-Lagrange equations. In order to derive the Euler-Lagrange equation of (8.4), we make the additional assumption that the norm $|\cdot|$ is strictly convex. Then by Remark 5.5 the duality map $J$ from $\left(\mathbb{R}^{n},|\cdot|^{*}\right)$ to $\left(\mathbb{R}^{n},|\cdot|\right)$ is single-valued.

Let $\phi \in C_{0}^{\infty}(U)$ be any "test function" and $t \in \mathbb{R}$. Since

$$
I_{p}\left(u_{p}\right) \leq I_{p}\left(u_{p}+t \phi\right)
$$

that is, the function $t \mapsto I_{p}\left(u_{p}+t \phi\right)$ has a minimum at $t=0$, we infer

$$
\int_{U}\left(\left|D u_{p}\right|^{*}\right)^{p-1}\left\langle J\left(D u_{p}\right), D \phi\right\rangle d x=0 .
$$

Thus $u_{p}$ satisfies the generalized $p$-Laplace equation

$$
-\operatorname{div}\left(\left(|D v|^{*}\right)^{p-1} J(D v)\right)=0
$$

in the sense of distributions. Observe that by approximation, (8.11) holds also for any $\phi \in W_{0}^{1, p}(U)$.

If the dual norm $|\cdot|^{*}$ is sufficiently smooth (and $p \geq 2$ ), any $p$-harmonic function $u$ on $U$ satisfies (8.12) in the viscosity sense. To see this, let $\varphi \in C^{2}(V), V \subset U$ open, be such that $-\operatorname{div}\left(\left(|D \varphi|^{*}\right)^{p-1} J(D \varphi)\right)>0$ in $V$. We want to show that $u-\varphi$ does not have any strict local maximum points in $V$. Suppose this is not the case, and let $z \in V$ be a strict maximum point such that $u(z)=\varphi(z)$. Let $\varphi_{\varepsilon}=\varphi-\varepsilon$ 
for a small $\varepsilon>0$. Then there is an open subset $V_{\epsilon} \subset V$ containing $z$ so that $\varphi_{\varepsilon}<u$ on $V_{\varepsilon}$ and $\varphi_{\varepsilon}=u$ on $\partial V_{\varepsilon}$. By using integration by parts and convexity, we obtain

$$
\begin{aligned}
0 & <\int_{V_{\varepsilon}}-\operatorname{div}\left(\left(\left|D \varphi_{\varepsilon}\right|^{*}\right)^{p-1} J\left(D \varphi_{\varepsilon}\right)\right)\left(u-\varphi_{\varepsilon}\right) d x \\
& =\int_{V_{\varepsilon}}\left(\left|D \varphi_{\varepsilon}\right|^{*}\right)^{p-1}\left\langle J\left(D \varphi_{\varepsilon}\right), D\left(u-\varphi_{\varepsilon}\right)\right\rangle d x=\left.\frac{d}{d t} I_{p}\left(\varphi_{\varepsilon}+t\left(u-\varphi_{\varepsilon}\right)\right)\right|_{t=0} \\
& \leq I_{p}(u)-I_{p}\left(\varphi_{\varepsilon}\right)=I_{p}(u)-I_{p}(\varphi),
\end{aligned}
$$

which clearly contradicts the fact that $u$ is $p$-harmonic. Hence $u-\varphi$ does not have any local maximum points in $V$, and $u$ must be a viscosity solution to (8.12).

Finally, let us formally derive the infinity Laplace equation (4.18) as a limit, as $p \rightarrow \infty$, of the $p$-Laplace equations. If $u_{p} \in C^{2}(U)$ satisfies (8.12), then

$$
-\left(\left|D u_{p}\right|^{*}\right)^{p-1} \operatorname{div} J\left(D u_{p}\right)-(p-1)\left(\left|D u_{p}\right|^{*}\right)^{p-2}\left\langle D^{2} u_{p} J\left(D u_{p}\right), J\left(D u_{p}\right)\right\rangle=0,
$$

which transforms into

$$
-\left\langle D^{2} u_{p} J\left(D u_{p}\right), J\left(D u_{p}\right)\right\rangle=\frac{1}{p-1}\left|D u_{p}\right|^{*} \operatorname{div} J\left(D u_{p}\right) .
$$

Assuming that $u_{p} \rightarrow u$ in $C^{2}(U)$ as $p \rightarrow \infty$, we obtain

$$
-\left\langle D^{2} u J(D u), J(D u)\right\rangle=0,
$$

that is, the limit function $u$ satisfies the infinity Laplace equation on $U$. This passage to the limit is routine in the theory of viscosity solutions, and we leave the rigorous proof of it as an exercise for the reader.

8.5. Notes. Approximation of the Lipschitz extension problem by the sequence of functionals (8.4) as $p \rightarrow \infty$ and taking a limit of the corresponding Euler-Lagrange equations were first proposed by Aronsson [5] and made completely rigorous, in case of the Euclidean norm, in 18. Since then these ideas have been successfully employed in various settings and forms, e.g., in [13, [15], 19], [20, [39], [44, [47, [48, 50, 51, 54, [59, 60, and 64. The facts about Sobolev spaces and functional analysis needed in order to prove the existence of $p$-harmonic functions by the direct method in calculus of variations can be found in many textbooks, see, e.g., 36] and 38. For a more detailed account of the theory of $p$-harmonic functions we refer the reader to [43]. The arguments in Sections 8.3 and 8.4 follow mostly that in [18]; see also [47] and [48].

\section{Appendix: Absolute minimizers in General Metric SpaCeS}

Throughout the main text of this paper, we have confined ourselves to investigating the Lipschitz extension problem only in the normed space $\left(\mathbb{R}^{n},|\cdot|\right)$. Yet it is clear that the problem itself makes sense and is interesting in a much wider context. For example, one can study various classes of Lipschitz extensions of a given function $f: A \rightarrow \mathbb{R}$, where $A$ is a closed subset of some metric space $(X, d)$, and try to develop a theory similar to what was described above in this generality. On the other hand, it is also worthwhile to analyze how general and versatile the ideas and methods we have used in the main text really are. The purpose of this appendix is, roughly speaking, to combine these two goals. In particular, we will sketch how a variant of the scheme that led to the existence in $\mathbb{R}^{n}$ can be used to accomplish an analogous result in the case of a fairly general metric space $(X, d)$. In 
this connection, we focus on the existence theory, since uniqueness and regularity cause difficulties already in $\left(\mathbb{R}^{n},|\cdot|\right)$.

First, let us stay for a while in $\mathbb{R}^{n}$ and review which properties of norms were needed in proving the existence result, Theorem 3.1. That is, we want to know when the same proof yields an absolute minimizer if the norm $|\cdot|$ is being replaced everywhere by a metric $d$. For starters, we note that a major role was played by the fact that the comparison with cones property characterizes the set of absolute minimizers, which in turn contains all cones whose vertex is outside the open set under consideration. In $\left(\mathbb{R}^{n}, d\right)$, however, cones do not always enjoy comparison with cones.

Example 9.1. In the case of the "snowflake" $\left(\mathbb{R},|\cdot|^{\alpha}\right), 0<\alpha<1$, the cones are of the form

$$
C(x)=b+a\left|x-x_{0}\right|^{\alpha}, \quad a, b \in \mathbb{R} .
$$

They do not enjoy comparison with cones, not even if we narrow down to cones whose slope is, say, nonnegative. To see this, let

$$
\begin{aligned}
& C_{0}(x)=|x|^{\alpha}, \\
& C_{1}(x)=a|x+1|^{\alpha}+b,
\end{aligned}
$$

where $a>1$ and $b<0$ will be determined below. We are looking for an open interval $I \subset] 0, \infty\left[\right.$ such that $C_{0}=C_{1}$ at the end points of $I$, but $C_{0}(x)>C_{1}(x)$ for all $x \in I$. First, we have $C_{0}(0)<C_{1}(0)$ if and only if $b>-a$. On the other hand, $C_{0}(1)>C_{1}(1)$ if $b<1-a 2^{\alpha}$. So we choose

$$
1<a<\left(2^{\alpha}-1\right)^{-1}
$$

then $-a<1-a 2^{\alpha}$, and hence it is possible to find $b$ satisfying

$$
-a<b<1-a 2^{\alpha} \text {. }
$$

These choices yield $C_{0}(0)<C_{1}(0)$ and $C_{0}(1)>C_{1}(1)$, and since $a>1, C_{1}(x)$ is larger than $C_{0}(x)$ for all $x$ sufficiently large. This shows that the interval $I$ with the desired properties can be found, and because of that, cones do not enjoy comparison with cones.

A quick look at Section 1.4 reveals that in $\left(\mathbb{R}^{n},|\cdot|\right)$, one thing we employed in proving that cones enjoy comparison with cones was the existence of geodesics penetrating through a given open set. This property holds for several metrics $d$ in $\mathbb{R}^{n}$ that are not induced by a norm, and it can be used for proving a statement analogous to Proposition 2.1. After that, one could follow through the proofs in Section 3 almost verbatim and establish existence. On the other hand, the "snowflake" in Example9.1does not contain any nonconstant curves of finite length and therefore does not have any nontrivial geodesics. If $X \neq \mathbb{R}^{n}$, the situation of course becomes much more complicated. New difficulties with the cones occur already in the following simple example.

Example 9.2. Consider the unit sphere $S^{n-1}$ of $\mathbb{R}^{n}$,

$$
S^{n-1}=\left\{x \in \mathbb{R}^{n}: \sum_{i=1}^{n} x_{i}^{2}=1\right\},
$$

equipped with the natural geodesic metric $d$. In this metric space, nonconstant cones are not absolutely minimizing. To see this, let $C(x)=b+a d\left(x, x_{0}\right)$ be a cone 
function. We may assume, without loss of generality, that $b=0, a=1$ and $x_{0}$ is the "north-pole" $(0, \ldots, 0,1) \in S^{n-1}$. If we choose $V$ to be the southern hemisphere, then $C$ is constant on $\partial V$, which is just the equator. Clearly

$$
\mathrm{L}_{C}(V)=1 \neq 0=\mathrm{L}_{C}(\partial V) .
$$

This reasoning shows also that the comparison with cones property does not characterize absolute minimizers, because constant functions, which are of course absolute minimizers, do not enjoy comparison with cones.

We regard the difficulties that emerged in the above example as mostly technical. One can easily adjust the notion of comparison with cones so that it takes into account the second singularity a cone has at the antipodal point relative to its vertex and then proceed. However, this is by no means the only cautionary example one has to deal with. As explained earlier, we are interested in finding a unified approach that is applicable in a quite general setting without any case-specific changes. One possibility is to use the McShane-Whitney extensions, and in the following we briefly describe this technique. We do not intend to formulate and prove the most general results possible, but our aim is to give the reader a rough idea of the method.

Let us begin by fixing the setting and introducing some terminology. Let $U$ be a proper open subset of a path-connected metric space $(X, d)$, and suppose $f: \partial U \rightarrow \mathbb{R}$ is a given Lipschitz continuous function; that is, there is $L<\infty$ such that

$$
|f(x)-f(y)| \leq L d(x, y) \text { for all } x, y \in \partial U .
$$

As before, we are interested in finding the "best" possible extension of $f$ to $U$. In the definition of absolute minimizers below, we have waived the requirement that the optimality of the Lipschitz constant is to be tested only in subsets with compact closure. This choice fits better to the very general framework, since now we do not need to worry about what compact sets could look like in different situations. As a drawback we can handle, in general, only Lipschitz boundary data.

Definition 9.3. We say that $u \in \operatorname{AM}(U)$ if $u$ is Lipschitz continuous in $\bar{U}$ and

$$
\mathrm{L}_{u}(V)=\mathrm{L}_{u}(\partial V)
$$

for every $V \subset U$.

Observe that since the space $(X, d)$ is assumed to be path-connected, every proper open subset of $X$ has nonempty boundary. Let $\gamma:[0,1] \rightarrow X$ be a continuous path. We define its length $\ell(\gamma)$ by

$$
\ell(\gamma):=\sup \left\{\sum_{i=0}^{k-1} d\left(\gamma\left(t_{i}\right), \gamma\left(t_{i+1}\right)\right): 0=t_{0}<t_{1}<\cdots<t_{k}=1, k \in \mathbb{N}\right\}
$$

and call $\gamma$ rectifiable if its length is finite. The metric space $(X, d)$ is called a length space, if

$$
d(x, y)=\inf \{\ell(\gamma): \gamma:[0,1] \rightarrow X \text { continuous, } \gamma(0)=x, \gamma(1)=y\}
$$

for all $x, y \in X$. In a length space, Lipschitz functions have a useful "glueing" property: if $v: U \rightarrow \mathbb{R}$ satisfies $\mathrm{L}_{v}(V) \leq L$ and $\mathrm{L}_{v}(\bar{U} \backslash V) \leq L$ for some open $V \subset U$, then $\mathrm{L}_{v}(U) \leq L$. This is not true in general if the space is not a length space; see [49]. The notion of "comparison with cones" played a central role in the 
proof of the existence result in Section [3. In the current framework, we replace cones by the upper and lower McShane-Whitney extensions

$$
\begin{aligned}
& \Lambda(f)(x):=\sup _{y \in \partial V}\left(f(y)-\mathrm{L}_{f}(\partial V) d(x, y)\right), \\
& \Psi(f)(x):=\inf _{y \in \partial V}\left(f(y)+\mathrm{L}_{f}(\partial V) d(x, y)\right) .
\end{aligned}
$$

They have the same properties in any metric space as in $\mathbb{R}^{n}$; that is, they are Lipschitz constant preserving extensions of $f$ and $\Lambda(f) \leq u \leq \Psi(f)$ if $u$ is any other Lipschitz constant preserving extension. In what follows, we will employ especially the latter property many times.

Definition 9.4. Let $u$ be Lipschitz continuous in $\bar{U}$. We say that $u$ enjoys comparison with the upper extensions in $U$ (abbreviated $u \in \operatorname{CUE}(U))$ if $u \leq \Psi\left(\left.u\right|_{\partial V}\right.$ ) in $V$ for every open $V \subset U$. Similarly, we say that $u$ enjoys comparison with the lower extensions in $U(u \in \operatorname{CLE}(U))$ if $u \geq \Lambda\left(\left.u\right|_{\partial V}\right)$ in $V$ for every $V \subset U$.

Observe that in the case $(X, d)=\left(\mathbb{R}^{n},|\cdot|\right)$ we have $\operatorname{CCA}(U)=\operatorname{CUE}(U)$ by Proposition 4.4. The counterpart for Proposition 2.1 is easily obtained.

Theorem 9.5. Let $U \subset X$ be open. Then $u \in \operatorname{AM}(U)$ if and only if $u$ enjoys comparison with the lower and the upper extensions.

Proof. Assume first that $u \in \operatorname{AM}(U)$. Then $\mathrm{L}_{u}(V)=\mathrm{L}_{u}(\partial V)$ for each $V \subset U$, and hence $\Lambda\left(\left.u\right|_{\partial V}\right) \leq u \leq \Psi\left(\left.u\right|_{\partial V}\right)$ by the properties of McShane-Whitney extensions. Consequently, $u$ enjoys comparison with the lower and the upper extensions.

To prove the opposite direction, we may argue as in the proof of Proposition 2.1 First, since $u \in \operatorname{CUE}(U) \cap \operatorname{CLE}(U)$, for any $V \subset U$ and any $x \in V$, we have

$$
u(z)-\mathrm{L}_{u}(\partial V) d(x, z) \leq u(x) \leq u(z)+\mathrm{L}_{u}(\partial V) d(x, z)
$$

for all $z \in \partial V$, which implies $\mathrm{L}_{u}(\partial(V \backslash\{x\}))=\mathrm{L}_{u}(\partial V)$ for each $x \in U$. Using this twice yields

$$
\mathrm{L}_{u}(\partial(V \backslash\{x, y\}))=\mathrm{L}_{u}(\partial V)
$$

for any $x, y \in V$. Since $x, y \in \partial(V \backslash\{x, y\})$, we obtain $|u(x)-u(y)| \leq \mathrm{L}_{u}(\partial V) d(x, y)$, and hence $u \in \operatorname{AM}(U)$.

The existence theorem we prove reads as follows:

Theorem 9.6. Let $(X, d)$ be a length space and $U$ a proper open subset of $X$. If $f: \partial U \rightarrow \mathbb{R}$ is Lipschitz continuous, then there exists $u \in \operatorname{AM}(U)$ such that $u=f$ on $\partial U$ and $\mathrm{L}_{u}(U)=\mathrm{L}_{f}(\partial U)$.

The general strategy for proving Theorem 9.6 is more or less the same as in Section 3 . We define

$$
u(x):=\sup \left\{v(x): \Lambda(f) \leq v \leq \Psi(f), v \in \operatorname{CUE}(U) \text { and } \mathrm{L}_{v}(U)=\mathrm{L}_{f}(\partial U)\right\} .
$$

The reader can check that the set of $v$ 's on the right is nonempty, because $\Lambda(f)$ satisfies all the requirements. Thus $u$ is well defined, and it is easy to see that $u=f$ on $\partial U$ and $\mathrm{L}_{u}(U)=\mathrm{L}_{f}(\partial U)$. In other words, the only thing that requires a proof is $u \in \operatorname{AM}(U)$. For this, we need to obtain "length space versions" of Lemma 2.12 and Lemma 3.3 . 
Lemma 9.7. Suppose $\mathcal{F}$ is a uniformly Lipschitz continuous family of functions which enjoy comparison with upper extensions in $U$, and let

$$
h(x)=\sup _{v \in \mathcal{F}} v(x) .
$$

Then $h$ enjoys comparison with upper extensions in $U$.

Proof. It is clearly enough to show that $v \leq \Psi\left(\left.h\right|_{\partial V}\right)$ for every $v \in \mathcal{F}$ and $V \subset U$. Let us therefore fix $V$ and $v$ and consider the set

$$
D=\left\{x \in V: v(x)>\Psi\left(\left.h\right|_{\partial V}\right)(x)\right\} .
$$

Our aim is to show that this set is necessarily empty. To this end, we define

$$
w(x)= \begin{cases}\Psi\left(\left.v\right|_{\partial D}\right)(x), & \text { if } x \in D, \\ \Psi\left(\left.h\right|_{\partial V}\right)(x), & \text { if } x \in V \backslash D .\end{cases}
$$

Since $v=\Psi\left(\left.h\right|_{\partial V}\right)$ on $\partial D$, we have

$$
\mathrm{L}_{w}(D)=\mathrm{L}_{v}(\partial D) \leq \mathrm{L}_{\Psi\left(\left.h\right|_{\partial V}\right)}(V) .
$$

On the other hand,

$$
\mathrm{L}_{w}(V \backslash D) \leq \mathrm{L}_{\Psi\left(\left.h\right|_{\partial V}\right)}(V) .
$$

Since $(X, d)$ is a length space, (9.2) and (9.3) together imply that

$$
\mathrm{L}_{w}(V) \leq \mathrm{L}_{\Psi\left(\left.h\right|_{\partial V}\right)}(V)=\mathrm{L}_{h}(\partial V) .
$$

Thus $w$ is a Lipschitz extension of $h$ to $V$ and because $\Psi\left(\left.h\right|_{\partial V}\right)$ is the largest such extension, $w \leq \Psi\left(\left.h\right|_{\partial V}\right)$ in $V$. In particular, since $v$ enjoys comparison with upper extensions,

$$
v \leq \Psi\left(\left.v\right|_{\partial D}\right)=w \leq \Psi\left(\left.h\right|_{\partial V}\right)
$$

in $D$. This shows that $D$ must be empty, and we are done.

Lemma 9.8. Suppose u enjoys comparison with upper extensions in $U$ but $u$ does not enjoy comparison with lower extensions in $U$. Then there exists a Lipschitz continuous function $\hat{u}$ and a nonempty proper subset $W \subset U$ such that $\hat{u} \in \operatorname{CUE}(U)$,

$$
\hat{u}=u \text { on } U \backslash W \text { and } \hat{u}>u \text { on } W .
$$

Proof. Let $V \subset U$ be such that

$$
W=\left\{x \in V: u(x)<\Lambda\left(\left.u\right|_{\partial V}\right)(x)\right\}
$$

is not empty and set

$$
\hat{u}(x)= \begin{cases}\Lambda\left(\left.u\right|_{\partial W}\right)(x) & \text { if } x \in W, \\ u(x) & \text { if } x \in U \backslash W .\end{cases}
$$

Using the facts that $(X, d)$ is a length space and $\Lambda\left(\left.u\right|_{\partial V}\right)$ is the smallest Lipschitz extension, with the optimal Lipschitz constant, of $u$ to $V$, and arguing as in the proof of Lemma 9.7 above, we see that $\Lambda\left(\left.u\right|_{\partial W}\right) \geq \Lambda\left(\left.u\right|_{\partial V}\right)$ in $W$. Thus we need to prove only that $\hat{u}$ enjoys comparison with upper extensions. To this end, we argue by contradiction and suppose that there exists $\hat{V} \subset U$ such that

$$
\hat{W}=\left\{x \in \hat{V}: \hat{u}(x)>\Psi\left(\left.\hat{u}\right|_{\partial \hat{V}}\right)(x)\right\}
$$

is not empty. Since $(X, d)$ is a length space, we may as well assume that $\hat{V}=\hat{W}$.

To conclude the proof, observe first that $u \leq \Psi\left(\left.\hat{u}\right|_{\partial \hat{V}}\right)$ in $\hat{V}$. Indeed, if we let

$$
D=\left\{x \in \hat{V}: u(x)>\Psi\left(\left.\hat{u}\right|_{\partial \hat{V}}\right)\right\},
$$


then

$$
\mathrm{L}_{\Psi\left(\left.u\right|_{\partial D}\right)}(D)=\mathrm{L}_{u}(\partial D) \leq \mathrm{L}_{\Psi\left(\left.\hat{u}\right|_{\partial \hat{V}}\right)}(\hat{V}) \leq \mathrm{L}_{\hat{u}}(\partial \hat{V}),
$$

and we have by the "glueing argument" and the maximality of the extension $\Psi\left(\left.\hat{u}\right|_{\partial \hat{V}}\right)$ that $\Psi\left(\left.\hat{u}\right|_{\partial \hat{V}}\right) \geq \Psi\left(\left.u\right|_{\partial D}\right)$ in $D$. By the assumption that $u \in \operatorname{CUE}(U)$, this implies $u \leq \Psi\left(\left.\hat{u}\right|_{\partial \hat{V}}\right)$ in $\hat{V}$, which also shows that $\hat{V} \subset W$. But as $\Lambda\left(\left.u\right|_{\partial W}\right)$ enjoys comparison with upper extensions in $W$ and $\Psi\left(\left.\hat{u}\right|_{\partial \hat{V}}\right)=\Psi\left(\left.\Lambda\left(\left.u\right|_{\partial W}\right)\right|_{\partial \hat{V}}\right)$ in $\hat{V}$, we have

$$
\hat{u}=\Lambda\left(\left.u\right|_{\partial W}\right) \leq \Psi\left(\left.\hat{u}\right|_{\partial \hat{V}}\right)
$$

in $\hat{V}$, a contradiction. We conclude that $\hat{u}$ enjoys comparison with upper extensions in $U$.

Theorem 9.6 now follows immediately. Indeed, by Lemma 9.7, $u \in \operatorname{CUE}(U)$. If $u \notin \operatorname{CLE}(U)$, we could use Lemma 9.8 to construct, without changing the boundary values or increasing the Lipschitz constant, a function $\hat{u} \in \operatorname{CUE}(U)$ such that $\hat{u} \geq u$ and $\hat{u}(x)>u(x)$ for some $x \in U$, which obviously contradicts the definition of $u$. Hence $u \in \operatorname{CUE}(U) \cap \operatorname{CLE}(U)$, and it then follows from Theorem 9.5 that $u \in \operatorname{AM}(U)$.

9.1. Notes. Absolute minimizers in general metric spaces have been studied in [57] and 49. Both papers concentrate mainly on the existence, which is proved using Perron's method in conjunction with the ideas of Aronsson [3]- [5]. Our presentation above follows 49. In the special case of a sub-Riemannian setting, absolute minimizers and the associated generalized infinity Laplace equations have been considered in [19], 20, 21], 22] and 62]. The results obtained include uniqueness and equivalence of absolute minimizers and viscosity solutions of the infinity Laplace equation.

As explained in Section 8 an alternative way to show existence of absolute minimizers in $\left(\mathbb{R}^{n},|\cdot|\right)$ goes via approximation by $p$-harmonic functions. Due to the development of the theory of Sobolev spaces and nonlinear potential theory on metric measure spaces, it is undoubtedly possible to generalize this approach to this more abstract setting. However, such method would presumably produce "strong absolute minimizers" as in the Euclidean case whose relationship with the absolute minimizers defined in Definition 9.3 is not a priori clear.

\section{REFERENCES}

[1] Almansa, A., Échantillonage, interpolation et détection. Applications en imagerie satellitaire, Ph.D. thesis, E.N.S. de Cachan (2002).

[2] Aronsson, G., Hur kan en sandhög se ut? (What is the possible shape of a sandpile?) NORMAT, vol. 13 (1965), 41-44.

[3] Aronsson, G., Minimization problems for the functional $\sup _{x} F\left(x, f(x), f^{\prime}(x)\right)$, Ark. Mat. 6 (1965), 33-53. MR 0196551 (33:4738)

[4] Aronsson, G., Minimization problems for the functional $\sup _{x} F\left(x, f(x), f^{\prime}(x)\right)$. II., Ark. Mat. 6 (1966), 409-431. MR 0203541 (34:3391)

[5] Aronsson, G., Extension of functions satisfying Lipschitz conditions, Ark. Mat. 6 (1967), 551-561. MR 0217665 (36:754)

[6] Aronsson, G., On the partial differential equation $u_{x}{ }^{2} u_{x x}+2 u_{x} u_{y} u_{x y}+u_{y}{ }^{2} u_{y y}=0$, Ark. Mat. 7 (1968), 395-425. MR 0237962 (38:6239)

[7] Aronsson, G., Minimization problems for the functional $\sup _{x} F\left(x, f(x), f^{\prime}(x)\right)$. III, Ark. Mat. 7 (1969), 509-512. MR 0240690 (39:2035) 
[8] Aronsson, G., On certain singular solutions of the partial differential equation $u_{x}^{2} u_{x x}+2 u_{x} u_{y} u_{x y}+u_{y}^{2} u_{y y}=0$, Manuscripta Math. 47 (1984), no. 1-3, 133-151. MR 0744316 (85m:35011)

[9] Aronsson, G., Construction of singular solutions to the p-harmonic equation and its limit equation for $p=\infty$, Manuscripta Math. 56 (1986), no. 2, 135-158. MR 0850366 (87j:35070)

[10] Aronsson, G., Evans, L. C., And Wu, Y., Fast/slow diffusion and growing sandpiles, J. Differential Equations 131 (1996), no. 2, 304-335. MR 1419017 (97i:35068)

[11] Banach, S., Wstęp do teorii funkcji rzeczywistych. (Polish) [Introduction to the theory of real functions], Monografie Matematyczne, Warszawa-Wrocław, 1951. MR 0043161 (13:216a)

[12] BARles, G., AND BusCA, J. Existence and comparison results for fully nonlinear degenerate elliptic equations without zeroth-order term, Comm. Partial Diff. Equations 26 (2001), 23232337. MR 1876420 (2002k:35078)

[13] Barron, E. N., Jensen, R. R., And Wang, C. Y., The Euler equation and absolute minimizers of $L^{\infty}$ functionals, Arch. Ration. Mech. Anal. 157 (2001), no. 4, 255-283. MR 1831173 (2002m:49006)

[14] Barron, E. N., Jensen, R. R., And Wang, C. Y., Lower semicontinuity of $L^{\infty}$ functionals, Ann. Inst. H. Poincaré Anal. Non Linéaire 18 (2001), no. 4, 495-517. MR 1841130 (2002c:49020)

[15] Belloni, M., AND KaWOHL, B., The pseudo-p-Laplace eigenvalue problem and viscosity solutions as $p \rightarrow \infty$, ESAIM Control Optim. Calc. Var. 10 (2004), 28-52.

[16] Bhattacharya, T., An elementary proof of the Harnack inequality for non-negative infinitysuperharmonic functions, Electron. J. Differential Equations, No. 44 (2001), 8 pp. (electronic). MR 1836812 (2002b:35071)

[17] Bhattacharya, T., On the properties of $\infty$-harmonic functions and an application to capacitary convex rings, Electron. J. Differential Equations, No. 101 (2002), 22 pp. (electronic). MR 1938397 (2003j:35126)

[18] Bhattacharya, T., DiBenedetto, E., and Manfredi, J., Limits as $p \rightarrow \infty$ of $\Delta_{p} u_{p}=f$ and related extremal problems, Some topics in nonlinear PDEs (Turin, 1989). Rend. Sem. Mat. Univ. Politec. Torino 1989, Special Issue, 15-68 (1991). MR 1155453 (93a:35049)

[19] Bieske, T., On $\infty$-harmonic functions on the Heisenberg group, Comm. Partial Differential Equations 27 (2002), no. 3-4, 727-761. MR 1900561 (2003g:35033)

[20] Bieske, T., Viscosity solutions on Grushin-type planes, Illinois J. Math. 46 (2002), no. 3, 893-911. MR 1951247 (2003k:35037)

[21] Bieske, T., Lipschitz extensions on generalized Grushin spaces, Michigan Math. J. (to appear).

[22] Bieske, T., And Capogna, L., The Aronsson-Euler equation for absolutely minimizing Lipschitz extensions with respect to Carnot-Carathéodory metrics, Trans. Amer. Math. Soc. (to appear).

[23] Caffarelli, L., And Cabre, J., Fully Nonlinear Elliptic Equations, American Mathematical Society, Colloquium Publications vol. 43, Providence, 1995. MR 1351007 (96h:35046)

[24] CAO, F., Absolutely minimizing Lipschitz extension with discontinuous boundary data, C. R. Acad. Sci. Paris Sér. I Math. 327 (1998), no. 6, 563-568. MR 1650611 (99h:49042)

[25] Caselles, V., Morel, J.-M., And Sbert, C., An axiomatic approach to image interpolation, IEEE Trans. Image Process. 7 (1998), no. 3, 376-386. MR 1669524 (2000d:94001)

[26] Champion, T., And De Pascale, L., A principle of comparison with distance functions for absolute minimizers, preprint.

[27] Champion, T., De Pascale, L., and Prinari, F., Г-convergence and absolute minimizers for supremal functionals, ESAIM Control Optim. Calc. Var. (to appear).

[28] Crandall, M. G., An efficient derivation of the Aronsson equation, Arch. Rational Mech. Anal. 167 (2003), 271-279. MR 1981858 (2004b:35053)

[29] Crandall, M. G., Evans, L. C., And Gariepy, R. F., Optimal Lipschitz extensions and the infinity Laplacian, Calc. Var. Partial Differential Equations 13 (2001), no. 2, 123-139. MR 1861094 (2002h:49048)

[30] Crandall, M. G., And Evans, L. C., A remark on infinity harmonic functions, Proceedings of the USA-Chile Workshop on Nonlinear Analysis (Viã del Mar-Valparaiso, 2000), 123-129 (electronic), Electron. J. Differ. Equ. Conf., 6, Southwest Texas State Univ., San Marcos, TX, 2001. MR 1804769 (2001j:35076) 
[31] Crandall, M. G., and Lions, P. L., Viscosity solutions of Hamilton-Jacobi equations, Trans. Amer. Math. Soc. 277 (1983), 1-42. MR 0690039 (85g:35029)

[32] Crandall, M. G., Ishit, H., And Lions, P. L., User's guide to viscosity solutions of second-order partial differential equations, Bull. Amer. Math. Soc. (N.S.) 27 (1992), 1-67. MR 1118699 (92j:35050)

[33] Crandall, M. G., And Zhang, J., Another way to say harmonic, Trans. Amer. Math. Soc. 355 (2003), 241-263. MR 1928087 (2003k:35062)

[34] Czipszer, J., And GehÉr, L., Extension of functions satisfying a Lipschitz condition, Acta Math. Acad. Sci. Hungar. 6 (1955), 213-220. MR0071493 (17:136b)

[35] Evans, L. C., Estimates for smooth absolutely minimizing Lipschitz extensions, Electron. J. Differential Equations 1993, No. 03, approx. 9 pp. (electronic only). MR 1241488 (94k:35117)

[36] Evans, L. C., And Gariepy, R. F., Measure Theory and Fine Properties of Functions, 1999, CRC Press, Boca Raton, Florida. MR 1158660 (93f:28001)

[37] Federer, H., Geometric Measure Theory, Springer-Verlag, New York, 1969. MR 0257325 (41:1976)

[38] Friedman, A., Foundations of Modern Analysis, Dover Publications, Inc., New York, 1982. MR 0663003 (83h:46001)

[39] Fukagai, N., Ito, M., And Narukawa, K., Limit as $p \rightarrow \infty$ of $p$-Laplace eigenvalue problems and $L^{\infty}$-inequality of the Poincaré type, Differential Integral Equations 12 (1999), no. 2, 183206. MR 1672746 (99m:35058)

[40] Gaspari, T., The infinity Laplacian in infinite dimensions, Calc. Var. Partial Differential Equations (to appear).

[41] Gilbarg, D., and Trudinger, N. S., Elliptic Partial Differential Equations of Second Order, Reprint of the 1998 edition. Classics in Mathematics, Springer-Verlag, Berlin, 2001. MR 1814364 (2001k:35004)

[42] Giusti, E., Direct Methods in the Calculus of Variations, World Scientific Publishing Co., Inc., River Edge, NJ, 2003. MR 1962933 (2004g:49003)

[43] Heinonen, J., Kilpeläinen, T., And Martio, O., Nonlinear Potential Theory of Degenerate Elliptic Equations, The Clarendon Press, Oxford University Press, New York, 1993. MR 1207810 (94e:31003)

[44] IshiBASHI, T., AND KoIKE, S., On fully nonlinear PDEs derived from variational problems of $L^{p}$ norms, SIAM J. Math. Anal. 33 (2001), no. 3, 545-569 (electronic). MR 1871409 (2002j:49037)

[45] Janfalk, U., Behaviour in the limit, as $p \rightarrow \infty$, of minimizers of functionals involving $p$ Dirichlet integrals, SIAM J. Math. Anal. 27 (1996), no. 2, 341-360. MR 1377478 (96m:49023)

[46] JENSEN, R. R., The maximum principle for viscosity solutions of fully nonlinear second order partial differential equations, Arch. Rational Mech. Anal. 101 (1988), no. 1, 1-27. MR 0920674 (89a:35038)

[47] JENSEN, R. R., Uniqueness of Lipschitz extensions: minimizing the sup norm of the gradient, Arch. Rational Mech. Anal. 123 (1993), no. 1, 51-74. MR 1218686 (94g:35063)

[48] Juutinen, P., Minimization problems for Lipschitz functions via viscosity solutions, Ann. Acad. Sci. Fenn. Math. Diss. No. 115 (1998), 53 pp. MR 1632063 (2000a:49055)

[49] Juutinen, P., Absolutely minimizing Lipschitz extensions on a metric space, Ann. Acad. Sci. Fenn. Math. 27 (2002), no. 1, 57-67. MR 1884349 (2002m:54020)

[50] Juutinen, P., Lindqvist, P., And Manfredi, J., The $\infty$-eigenvalue problem, Arch. Ration. Mech. Anal. 148 (1999), no. 2, 89-105. MR 1716563 (2000g:35047)

[51] Lindqvist, P., AND Manfredi, J., The Harnack inequality for $\infty$-harmonic functions, Electron. J. Differential Equations (1995), No. 04, approx. 5 pp. MR 1322829 (96b:35025)

[52] Lindqvist, P., And Manfredi, J., Note on $\infty$-superharmonic functions, Rev. Mat. Univ. Complut. Madrid 10 (1997), no. 2, 471-480. MR 1605682 (98k:35043)

[53] Lindqvist, P., Manfredi, J., And Saksman, E., Superharmonicity of nonlinear ground states, Rev. Mat. Iberoamericana 16 (2000), no. 1, 17-28. MR 1768532 (2001f:35133)

[54] Manfredi, J., Petrosyan, A., and Shahgholian, H., A free boundary problem for $\infty$ Laplace equation, Calc. Var. Partial Differential Equations 14 (2002), no. 3, 359-384. MR 1899452 (2003a:35212)

[55] McShane, E. J., Extension of range of functions, Bull. Amer. Math. Soc. 40 (1934), 837-842. 
[56] Mil'man, V. A., Lipschitz extensions of linearly bounded functions (Russian), Mat. Sb. 189 (1998), no. 8, 67-92; translation in Sb. Math. 189 (1998), no. 7-8, 1179-1203. MR 1669631 (2000b:46043)

[57] Mil'man, V. A., Absolutely minimal extensions of functions on metric spaces (Russian), Mat. Sb. 190 (1999), no. 6, 83-110; translation in Sb. Math. 190 (1999), no. 5-6, 859-885. MR 1719573 (2000j:41041)

[58] Reich, S., Review of "Geometry of Banach spaces, duality mappings and nonlinear problems" by Ioana Cioranescu, Bull. Amer. Math. Soc. 26 (N.S.) (1992), 367-370.

[59] Rosset, E., A lower bound for the gradient of $\infty$-harmonic functions, Electron. J. Differential Equations, No. 02 (1996), approx. 6 pp. (electronic). MR 1371219 (96k:35066)

[60] Rosset, E., Symmetry and convexity of level sets of solutions to the infinity Laplace's equation, Electron. J. Differential Equations, No. 34 (1998), 12 pp. (electronic). MR 1656591 (99j:35068)

[61] Savin, O., $C^{1}$ regularity for infinity harmonic functions in two dimensions, preprint.

[62] WANG, C., The Aronsson equation for absolute minimizers of $L^{\infty}$-functionals associated with vector fields satisfying Hörmander's condition, preprint (2003).

[63] Whitney, H., Analytic extensions of differentiable functions defined in closed sets, Trans. Amer. Math. Soc. 36 (1934), no. 1, 63-89. MR 1501735

[64] Wu, Y., Absolute minimizers in Finsler metrics, Ph. D. dissertation, UC Berkeley, 1995.

[65] YU, Y., Sufficiency of Aronsson-Euler equations without zeroth order terms, preprint.

Department of Mathematics, Linköping University, SE-581 83 Linköping, Sweden

E-mail address: guaro@mai.liu.se

Department of Mathematics, University of California, Santa Barbara, Santa BarBARA, CALIFORNIA 93106

E-mail address: crandall@math.ucsb.edu

Department of Mathematics and Statistics, P.O. Box 35, Fin-40014 University of JYVÄSKYlä, FinLAND

E-mail address: peanju@maths.jyu.fi 